André Luis Ferreira da Silva

Otimização Topológica de Estruturas de Pórtico Laminado Considerando Orientação de Fibra e Regime Linear 
André Luis Ferreira da Silva

\section{Otimização Topológica de Estruturas de Pórtico Laminado Considerando Orientação de Fibra e Regime Linear}

Dissertação de Mestrado apresentado à Escola Politécnica da Universidade de São Paulo para obtenção do Título de Mestre em Ciências. 
André Luis Ferreira da Silva

\title{
Otimização Topológica de Estruturas de Pórtico Laminado Considerando Orientação de Fibra e Regime Linear
}

\author{
Versão Corrigida
}

Dissertação de Mestrado apresentado à Escola Politécnica da Universidade de São Paulo para obtenção do Título de Mestre em Ciências.

Área de concentração:

Engenharia de Controle e Automação Mecânica (3152)

Orientador:

Prof. Dr. Emílio Carlos Nelli Silva 
Autorizo a reprodução e divulgação total ou parcial deste trabalho, por qualquer meio convencional ou eletrônico, para fins de estudo e pesquisa, desde que citada a fonte.

Este exemplar foi revisado e alterado em relação à versão original, sob responsabilidade única do autor e com a anuência de seu orientador.

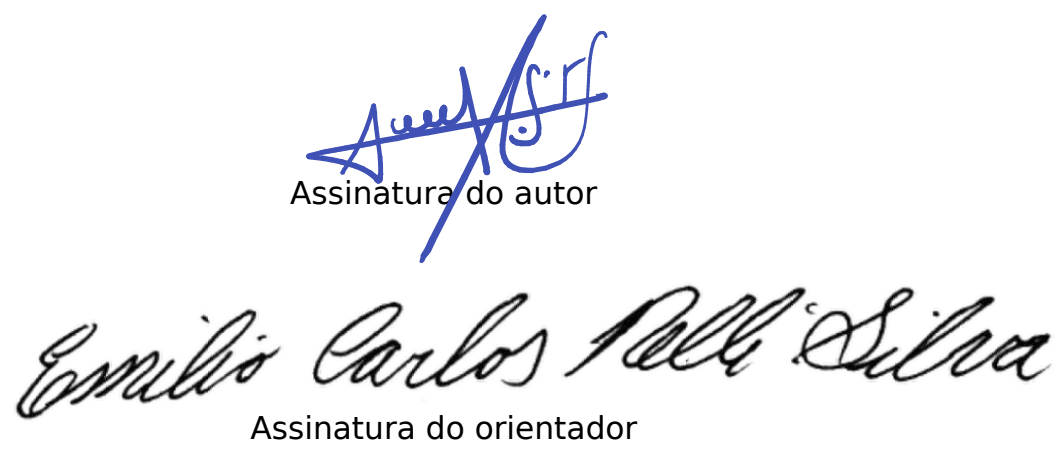

Catalogação-na-publicação

Silva, Andre Luis Ferreira da

Otimização Topológica de Estruturas de Pórtico Laminado Considerando Orientação de Fibra e Regime Linear / A. L. F. Silva -- São Paulo, 2020. $106 \mathrm{p}$.

Dissertação (Mestrado) - Escola Politécnica da Universidade de São Paulo. Departamento de Engenharia Mecatrônica e de Sistemas Mecânicos.

1.Otimização Topológica 2.Compósitos Reforçados com Fibras 3.MIAFIB 4.OFDN-m I.Universidade de São Paulo. Escola Politécnica. Departamento de Engenharia Mecatrônica e de Sistemas Mecânicos II.t. 
Silva, André Luis Ferreira da Silva. Otimização Topológica de Estruturas de Pórtico Laminado Considerando Orientação de Fibra e Regime Linear. 2020. 107 p. Dissertação (Mestrado) - Escola Politécnica, Universidade de São Paulo, São Paulo, 2020.

Aprovado em:

\section{Banca Examinadora}

Prof. Dr.

Instituição:

Julgamento:

Prof. Dr.

Instituição:

Julgamento:

Prof. Dr.

Instituição:

Julgamento: 
Dedico este trabalho aos meus pais e meu irmão pelas lições, apoio e incentivo ao longo de toda a minha vida. 


\section{AGRADECIMENTOS}

Ao meu orientador Emílio Carlos Nelli Silva por ter me dado a oportunidade, por acreditar em meu trabalho, por toda paciência, pela motivação e empenho para realização deste trabalho.

Aos meus pais Antônio Ferreira e Maria Aparecida e ao meu irmão Carlos Eduardo por todo amor e ensinamentos ao longo da minha vida.

A minha sobrinha Sophia Maria por me contagiar com sua alegria em todas as vezes que a via.

Aos membros do grupo de pesquisa do Laboratório de Otimização de Sistemas Multifísicos: Ruben, Paulo, Luís Sá, Luís Garcia, Diego Alonso, Diego Prado, Bruno, Mohammad, Ricardo Amigo, Ricardo Lahuerta, Hélio, Francisco e Gustavo por tudo que me ensinaram e por todas as vezes que me ajudaram.

A CAPES (Coordenação de Aperfeiçoamento de Pessoal de Nível Superior) e ao INCT-CEMTEC (Tecnologias Cimentícias Eco-eficientes Avançadas - Tecnologias Baseadas em Cimento) pelo apoio financeiro neste trabalho por meio da bolsa auxílio.

E por fim, a todos aqueles que contribuíram de forma direta ou indireta para a realização deste trabalho. 
"A humildade é o sólido fundamento de todas as virtudes". - Confúcio 


\section{RESUMO}

Este trabalho tem como objetivo desenvolver uma metodologia para projetar estruturas de materiais compósitos reforçados com fibras utilizando otimização topológica baseada em modelos de densidade e orientação de fibra. A utilização de materiais compósitos reforçados com fibras em aplicações de engenharia tem aumentado significativamente nas últimas décadas em função das vantagens que este tipo de material oferece. As propriedades desta classe de material dependem de como as fibras estão distribuídas em seu interior. Assim, melhorias no desempenho em estruturas fabricadas com compósitos reforçados com fibras podem sem alcançadas por meio da utilização de métodos de otimização, como a otimização topológica. O projeto de estruturas compósitas inicia-se com a modelagem estrutural utilizando o Método de Elementos Finitos para a solução das equações diferenciais. A determinação da orientação otimizada das fibras é feita utilizando os métodos Modelo de Interpolação Autopenalizável da Orientação da Fibra (MIAFIB), que considera o ângulo da fibra diretamente como uma variável de projeto e Otimização de Fibra por Distribuição Normal - modificado (OFDN-m), que considera um conjunto de ângulos candidatos e tem como objetivo determinar o ângulo otimizado para cada nó da malha de elementos finitos. A distribuição de material no domínio é realizada empregando-se o modelo Solid Isotropic Material with Penalization (SIMP). O problema de minimização da flexibilidade é implementado no ambiente FEniCS para a solução do problema de elementos finitos. Para o cálculo das sensibilidades e solução do problema de otimização, são utilizadas as bibliotecas libadjoint e IPOpt respectivamente. O filtro proposto por Lazarov, baseado em uma equação modificada de Helmholtz, é utilizado para tratar a descontinuidade das fibras e para tratar da dependência de malha e escala de cinza na distribuição de material. Uma restrição baseada no modelo de Borrvall é utilizada para garantir a continuidade e tratar o problema de acúmulo das fibras. São apresentados exemplos numéricos para verificar a generalidade dos métodos propostos, assim como um estudo da influência dos parâmetros empregados no filtro de Helmholtz.

Palavras-chave: Otimização Topológica, Compósitos Reforçados com Fibras, MIAFIB, OFDN-m 


\section{ABSTRACT}

This work aims to develop a methodology for designing fiber reinforced composite structures using topology optimization based on density and fiber orientation models. The use of composite materials reinforced with fibers in engineering applications has increased significantly in the last decades due to the advantages that this type of material offers. The properties of this material class depend on how the fibers are distributed. Thus, improvements in the performance of structures made of fiber-reinforced composites can be achieved by using optimization methods, such as, topology optimization. The design of composite structures begins with structural modeling using the Finite Element Method for the solution of differential equations. Determination of optimized fibers orientation is achieved by using two different models, the Self-Penalizable Interpolation Model for Fiber Orientation (SPIMFO) method, which considers the fiber angle directly as a design variable and the Normal Distribution Fiber Optimization - modified (NDFO-m), which considers a set of candidate angles and aims to determine which of these angles is the optimized angle for each node of the finite element mesh. The material distribution in the domain is carried out using the Solid Isotropic Material with Penalization (SIMP) model. The problem of compliance minimization is implemented in the FEniCS environment to determine the Finite Element problem solution. Calculation of sensitivities and solution of the optimization problem, are accomplished by using libadjoint and IPOpt libraries respectively. The filter proposed by Lazarov, based on a modified Helmholtz equation, is used to ensure the fiber continuity and treat the mesh dependency and grayscale problem in the material distribution. A constraint based on the Borrvall model is used to ensure continuity and address the fiber accumulation problem. Numerical examples are presented to verify the generality of the proposed methods, as well as a study of the influence of the parameters used in the Helmholtz filter.

Keywords: Topology Optimization, Fiber Reinfoced Materials, SPIMFO, NDFO-m 


\section{LISTA DE ILUSTRAÇÕES}

Figura 1.1 - Representação genérica de um compósito reforçado com fibras. . . . . 18

Figura 1.2 -Braço robótico para manufatura aditiva. . . . . . . . . . . . . . . . . . 19

Figura 1.3 - Representação do bico de impressão para concreto reforçado com fibras. 20

Figura 1.4-Procedimento de Otimização Topológica (OT) . . . . . . . . . . . . . . . 23

Figura 2.1 - Planos de simetria material em um material ortotrópico . . . . . . . . . 28

Figura 2.2 - Eixos globais e locais em um Compósitos Reforçados com Fibras (CRF) 30

Figura 2.3 - Convergência em função do refinamento de malha. . . . . . . . . . . . . 33

Figura 2.4 - Sólido genérico com um elemento tri-dimensional de 8 nós . . . . . . . 34

Figura 2.5 - Mapeamento entre o espaço de referência e o espaço Euclidiano. . . . . 35

Figura 2.6 - Elemento triangular com interpolação quadrática. . . . . . . . . . . . . . . 37

Figura 2.7 - Coordenadas naturais de um elemento tetraédrico de 4 nós. . . . . . . . 38

Figura 3.1 - Resultado de OT utilizando método das densidades. . . . . . . . . . . . 39

Figura 3.2 - Definição do domínio de projeto . . . . . . . . . . . . . . . . . . . . . . 40

Figura 3.3 - Influência do penalizador $p \ldots \ldots$. . . . . . . . . . . . . . . . . . . . . . 41

Figura 3.4 - Representação de um resultado com escala de cinza. . . . . . . . . . . . 42

Figura 3.5 - Influência da penalização na solução do problema de OT. . . . . . . . . . 42

Figura 3.6 - Padrão de instabilidade de tabuleiro em uma viga simplesmente apoiada. 43

Figura 3.7 - Efeito da dependência de malha . . . . . . . . . . . . . . . . . . . . . . 44

Figura 3.8 - Exemplo de não unicidade da solução. . . . . . . . . . . . . . . . . . . 45

Figura 3.9 - Efeito da alteração do parâmetro $r$ na solução da equação modificada de Helmholtz. . . . . . . . . . . . . . . . . . . . . . . . 47

Figura 3.10-Definição do ângulo $\theta \ldots \ldots \ldots$. . . . . . . . . . . . . . . . . 49

Figura 3.11-Efeitos causados pela alteração de $\theta_{c}$ e $p_{\theta}$. . . . . . . . . . . . . . . 50

Figura 3.12- $\theta$ exatamente entre o menor intervalo entre os ângulos candidatos . . . 50

Figura 3.13-Aproximação da funções seno e cosseno por séries de Taylor. . . . . . . 53

Figura 3.14-Continuidade das fibras . . . . . . . . . . . . . . . . . 53

Figura 3.15-Acúmulo de fibras . . . . . . . . . . . . . . . . . . . . . . 54

Figura 3.16-Escoamento em um tanque aberto . . . . . . . . . . . . . . . . . . . . 54

Figura 3.17-Influência do penalizador $q$ na função de interpolação $\alpha\left(\rho_{l}\right) \quad \ldots$. . . . . 56

Figura 4.1 - Definição do problema de maximização de rigidez em um material compósito utilizando OT . . . . . . . . . . . . . . . . . . . . . . . . . . . . . . . 58

Figura 5.1 - Funcionamento do compilador FEniCS Form Compiler (FFC) . . . . . . . 66

Figura 5.2-Diferentes modos para obtenção do código para implementação do sistema de equações adjuntas. . . . . . . . . . . . . . . . . . . . . 67

Figura 5.3 - Fluxograma de implementação de OT . . . . . . . . . . . . . . . . . . . . 69 
Figura 6.1 - Elementos tetraédricos com interpolação linear. . . . . . . . . . . . . . 71

Figura $6.2-$ Viga engastada. . . . . . . . . . . . . . . . . . . . . . . . . . . . 71

Figura 6.3 - Orientação otimizada das fibras para uma viga engastada. . . . . . . . . 72

Figura 6.4 - Convergência para a viga engastada. . . . . . . . . . . . . . . . . 73

Figura 6.5 - Resultado com distribuição nas direções de tensão principal. . . . . . . 74

Figura 6.6 - Convergências das funções objetivo considerando $r=15 \mathrm{~mm} \ldots 75$

Figura 6.7 -Viga simplesmente apoiada. . . . . . . . . . . . . . . . . . . 75

Figura 6.8 - Orientação otimizada para a viga simplesmente apoiada. . . . . . . . . 76

Figura 6.9 - Convergência para a viga simplesmente apoiada. . . . . . . . . . . . 77

Figura 6.10-Placa fixa com força fora do plano. . . . . . . . . . . . . . . . . 78

Figura 6.11-Orientação otimizada para placa com força fora do plano. . . . . . . . . 79

Figura 6.12-Convergência para a placa com força fora do plano. . . . . . . . . . . . 80

Figura 6.13-Elementos triangulares com interpolação linear. . . . . . . . . . . . . . 81

Figura 6.14-Domínio da viga em balanço. . . . . . . . . . . . . . . . . . . . . . . . . 81

Figura 6.15-Resultado otimizado para a viga em balanço. . . . . . . . . . . . . . . 82

Figura 6.16-Interpretação do resultado para a viga em balanço. . . . . . . . . . . . . . 83

Figura 6.17-Condições de contorno para o modelo de Borrvall no problema da viga em balanço. . . . . . . . . . . . . . . . . . 83

Figura 6.18-Resultado para a viga em balanço com restrição de continuidade. . . . . 85

Figura 6.19-Domínio da viga MBB . . . . . . . . . . . . . . . . . . . . . . . . . . 86

Figura 6.20-Resultado para a viga MBB . . . . . . . . . . . . . . . . . . 86

Figura 6.21-Condições de contorno para o modelo de Borrvall no problema da viga MBB. . . . . . . . . . . . . . . . . . . 86

Figura 6.22-Resultado para a viga MBB com restrição de continuidade. . . . . . . . . 87

Figura 6.23-Domínio da estrutura "L" . . . . . . . . . . . . . . . . . . . . . . . 88

Figura 6.24-Resultado para a estrutura em "L". . . . . . . . . . . . . . . . . . . . . . . 89

Figura 6.25-Condições de contorno para o modelo de Borrvall no problema da estrutura "L" . . . . . . . . . . . . . . . . . . . . . . . . . 89

Figura 6.26-Resultado para a estrutura "L" com restrição de continuidade. . . . . . . . 90

Figura 6.27-Convergências das funções objetivo. . . . . . . . . . . . . . . . . . . . . 92

Figura 6.28-Iterações com valores de função objetivo negativos no exemplo da estrutura "L" . . . . . . . . . . . . . . . . . . . . . . . . . . . . 92 


\section{ACRONYMS}

CRF Compósitos Reforçados com Fibras

EPT Estado Plano de Tensão

FFC FEniCS Form Compiler

IPOpt "Interior Point Optimizer"

MEF Método dos Elementos Finitos

MBG Métodos Baseados em Gradiente

MIAFIB Modelo de Interpolação Autopenalizável da Orientação da Fibra

ODM Otimização Discreta de Material

OT Otimização Topológica

OFDN Otimização de Fibra por Distribuição Normal

OFDN-m Otimização de Fibra por Distribuição Normal modificado

PCB Parametrização por Codificação Binária

PTV Princípio dos Trabalhos Virtuais

PFF Parametrização por Funções de Forma

SIMP "Solid Isotropic Material with Penalization"

UFC "Unified Form-Assembly Code"

UFL "Unified Form Language" 


\section{LISTA DE SÍMBOLOS}

\section{Símbolos Latinos}

\begin{tabular}{|c|c|}
\hline a & Variável de projeto genérica \\
\hline$\tilde{a}$ & Variável de projeto genérica filtrada \\
\hline$\hat{\mathbf{a}}$ & Vetor de variáveis de projetos nodais genéricas \\
\hline B & Matriz que relaciona deformação e deslocamento \\
\hline $\mathbf{C}$ & Tensor constitutivo \\
\hline$\overline{\mathbf{C}}$ & Tensor constitutivo transformado \\
\hline$\overline{\mathbf{C}}_{e}$ & Tensor constitutivo efetivo \\
\hline$\overline{\mathbf{C}}_{a d}$ & Conjunto de tensores constitutivos admissíveis \\
\hline $\mathbf{D}$ & Tensor da taxa de deformação \\
\hline$E$ & Módulo de Young \\
\hline $\mathbf{f}$ & Vetor de forças \\
\hline $\mathbf{f}^{B}$ & Forças de volume no sólido \\
\hline $\mathbf{f}_{l}^{B}$ & Forças de volume no fluido \\
\hline $\mathbf{f}^{S_{f}}$ & Forças superficiais \\
\hline$G$ & Módulo de cisalhamento \\
\hline h & Função de interpolação \\
\hline $\mathbf{H}$ & Matriz de interpolação \\
\hline I & Matriz identidade \\
\hline $\mathbf{J}$ & Operador Jacobiano \\
\hline $\mathbf{K}$ & Matriz de rigidez \\
\hline$L$ & Lagrangiano do problema de otimização \\
\hline$p$ & Penalizador do modelo SIMP \\
\hline $\bar{p}$ & Pressão \\
\hline$\delta \bar{p}$ & Função de teste para pressão \\
\hline$p_{\theta}$ & Penalizador do método OFDN-m \\
\hline$p_{\theta}^{\min }$ & Valor mínimo para o penalizador do método OFDN-m \\
\hline$p_{f}$ & Penalizador do MIAFIB \\
\hline$P$ & Função de barreira logarítmica \\
\hline $\mathbf{Q}$ & Tensor constitutivo para o estado plano de tensão \\
\hline$\overline{\mathbf{Q}}$ & Tensor constitutivo para o estado plano de tensão transformado \\
\hline$r$ & Parâmetro de comprimento do filtro de Helmholtz \\
\hline $\mathbf{r}_{B}$ & Vetor de forças de volume \\
\hline $\mathbf{r}_{C}$ & Vetor de cargas concentradas \\
\hline $\mathbf{r}_{2}$ & Vetor de tensões iniciais \\
\hline
\end{tabular}




$\begin{array}{ll}\mathbf{r}_{S} & \text { Vetor de forças superficiais } \\ \mathbf{R} & \text { Matriz de Reuter } \\ \mathbf{R}_{E P T} & \text { Matriz de Reuter para o estado plano de tensão } \\ s_{p}^{M I A F I B} & \text { Número de iterações para cada valor de penalizador no modelo } \\ & \text { MIAFIB } \\ \mathbf{s}_{f} & \text { Sensibilidade da função objetivo na forma discreta em relação a } \\ & \text { variável de projeto filtrada } \\ S & \text { Superfície do sólido } \\ S_{u} & \text { Superfície com deslocamentos prescritos } \\ S_{f} & \text { Superfícies sujeitas à aplicação de forças superficiais } \\ S_{l} & \text { Fronteiras do volume de controle } \\ \mathbf{S} & \text { Tensor de flexibilidade } \\ \mathbf{T} & \text { Matriz de transformação } \\ \mathbf{T}_{E P T} & \text { Matriz de transformação para o estado plano de tensão } \\ \mathbf{u} & \text { Vetor de deslocamentos } \\ \mathbf{u}^{S_{u}} & \text { Vetor de deslocamentos prescritos em } S_{u} \\ \hat{\mathbf{u}} & \text { Vetor de deslocamentos nodais } \\ v & \text { Função teste } \\ \overline{\mathbf{v}}_{l} & \text { Velocidade do fluido } \\ \delta \overline{\mathbf{v}}_{l} & \text { Função de teste de velocidade } \\ V & \text { Volume do sólido } \\ V_{l} & \text { Volume de controle } \\ V^{m a t} & \text { Subdomínio que representa a distribuição otimizada de material } \\ V & \text { Quantidade limite de material disponível } \\ \hat{w} & \text { Função de ponderação } \\ \mathbf{x} & \text { Vetor de coordenadas cartesianas } \\ & \end{array}$

continue... 


\section{Símbolos Gregos}

\begin{tabular}{|c|c|}
\hline$\alpha$ & Permeabilidade inversa \\
\hline$\underline{\alpha}$ & Valor mínimo de $\alpha$ \\
\hline $\bar{\alpha}$ & Valor máximo de $\alpha$ \\
\hline$\beta$ & Vetor limite para restrição de continuidade \\
\hline$\beta$ & Magnitude de $\beta$ \\
\hline$\gamma$ & Deformação de cisalhamento \\
\hline$\delta$ & Operador variacional \\
\hline$\delta \mathbf{u}$ & Deslocamentos virtuais \\
\hline$\delta \mathbf{u}^{i}$ & Deslocamentos virtuais equivalentes a carga concentrada $\mathbf{R}_{C}^{i}$ \\
\hline$\delta \mathbf{u}^{S_{f}}$ & Deslocamentos virtuais avaliados em $S_{f}$ \\
\hline$\delta W_{\text {ext }}$ & Trabalho virtual dos esforços externos \\
\hline$\delta W_{\text {int }}$ & Trabalho virtual dos esforços internos \\
\hline$\delta \varepsilon$ & Deformações virtuais \\
\hline$\Delta p$ & Incremento do penalizador $p$ \\
\hline$\varepsilon$ & Tensor das deformações \\
\hline$\varepsilon$ & Deformação normal \\
\hline$\zeta$ & Terceira coordenada natural \\
\hline$\eta$ & Segunda coordenada natural \\
\hline$\theta$ & Variável de orientação da fibra \\
\hline$\theta_{c}^{i}$ & Ângulo candidato $i$ \\
\hline$\hat{\theta}$ & Pseudo-orientação do modelo MIAFIB \\
\hline$\theta$ & Vetor de projeções de $\theta$ \\
\hline$\lambda$ & Vetor adjunto \\
\hline$\lambda_{i}$ & Multiplicadores de Lagrange \\
\hline$\mu$ & Parâmetro de barreira logarítmica \\
\hline$\mu_{l}$ & Viscosidade dinâmica \\
\hline$v$ & Coeficiente de Poisson \\
\hline$\xi$ & Primeira coordenada natural \\
\hline$\xi$ & Vetor de coordenadas naturais \\
\hline$\rho$ & Densidade do sólido \\
\hline $\bar{\rho}$ & Pseudo-Densidade \\
\hline$\rho_{0}$ & Densidade do fluido \\
\hline$\sigma$ & Tensão normal \\
\hline$\sigma$ & Tensor das tensões \\
\hline$\sigma_{l}$ & Tensor de tensões para o fluido \\
\hline$\sigma^{I}$ & Tensor das tensões iniciais \\
\hline$\tau$ & Tensão de cisalhamento \\
\hline
\end{tabular}


$\phi \quad$ Combinação entre função objetivo e função de barreira logarítmica

$\Psi \quad$ Energia de deformação específica 


\section{SUMÁRIO}

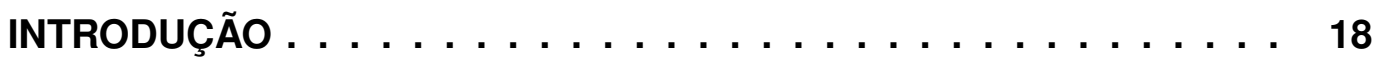

$1.1 \quad$ Compósitos reforçados com fibra $\ldots \ldots \ldots \ldots \ldots$

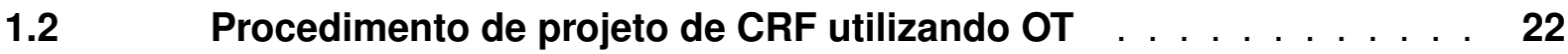

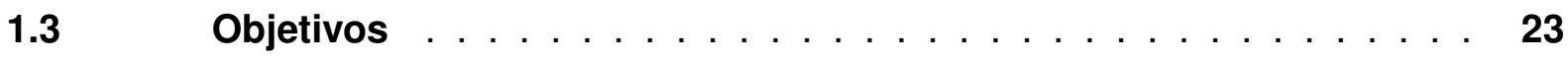

$1.4 \quad$ Motivação . . . . . . . . . . . . . . . . . . . 24

2 MODELAGEM DE COMPÓSITOS REFORÇADOS COM FIBRAS . . 25

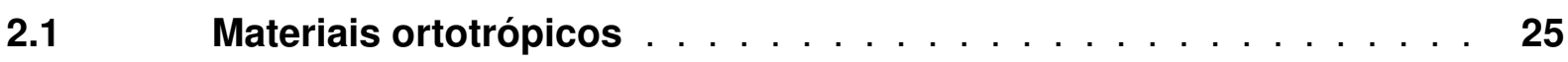

2.2 Estado plano de tensão para Compósitos Reforçados com Fibras . 29

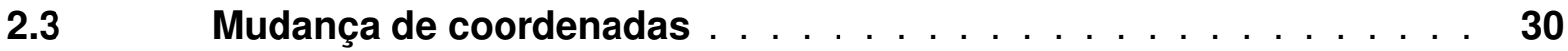

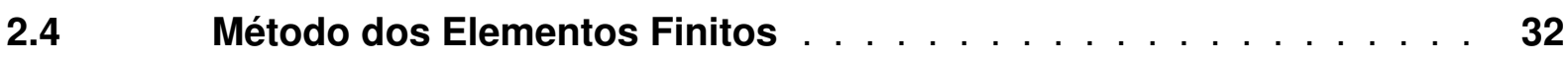

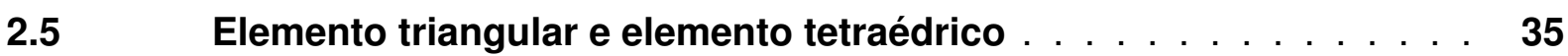

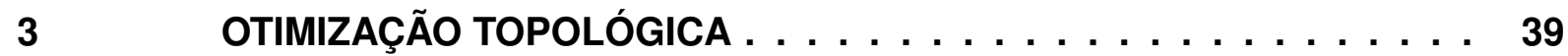

$3.1 \quad$ Modelo de Material SIMP $\ldots \ldots \ldots \ldots \ldots$

$3.2 \quad$ Instabilidade de tabuleiro $\ldots \ldots \ldots \ldots \ldots$

3.3 Dependência de malha $\ldots \ldots \ldots \ldots \ldots$

$3.4 \quad$ Métodos de filtragem $\ldots \ldots \ldots \ldots \ldots$

3.5 Modelos para otimização de orientação de fibras . . . . . . . . 48

3.5.1 Método Otimização de Fibra por Distribuição Normal modificado (OFDN-m) 48

3.5.2 Modelo de Interpolação Autopenalizável da Orientação da Fibra (MIAFIB) 51

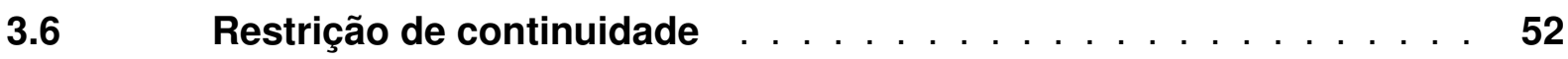

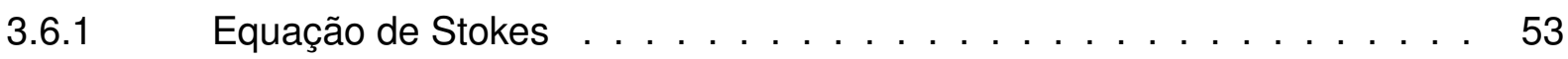

3.6.2 Modelo de Borrvall . . . . . . . . . . . . . . . . . . . . 55

3.6.3 Restrição baseada no modelo de Borrvall . . . . . . . . . . . . . 57

4 FORMULAÇÃO DO PROBLEMA DE OT PARA O PROJETO DE MATERIAIS COMPÓSITOS REFORÇADOS COM FIBRA . . . . . . . . . . 58

$4.1 \quad$ Formulação do problema para maximização da rigidez . . . . . . 58

$4.2 \quad$ Sensibilidades . . . . . . . . . . . . . . . . . . 59

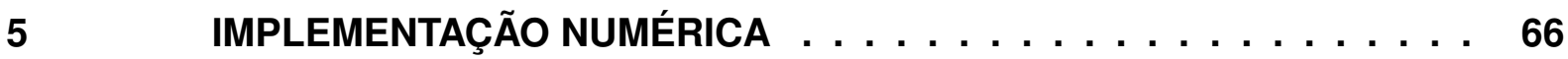

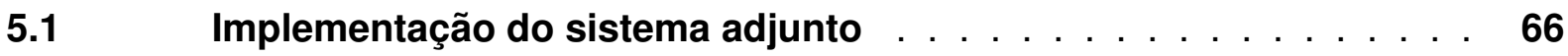

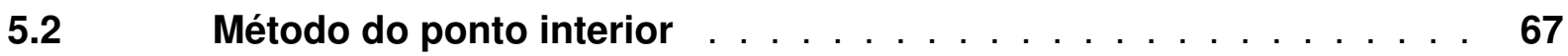

$5.3 \quad$ Implementação da 0 T $\ldots \ldots \ldots \ldots \ldots$ 
RESULTADOS . . . . . . . . . . . . . . . . . . . . . 70

$6.1 \quad$ Resultados de OT aplicada a orientação de fibras . . . . . . . . . . 70

$6.1 .1 \quad$ Viga engastada . . . . . . . . . . . . . . . . . . 71

$6.1 .2 \quad$ Viga simplesmente apoiada . . . . . . . . . . . . . . . 74

6.1.3 Placa com força fora do plano . . . . . . . . . . . . . . . . . 77

6.2 Resultados de OT aplicada simultaneamente a distribuição de material e orientação de fibras . . . . . . . . . . . . . . . . . . . 79

$6.2 .1 \quad$ Viga em balanço . . . . . . . . . . . . . . . 80

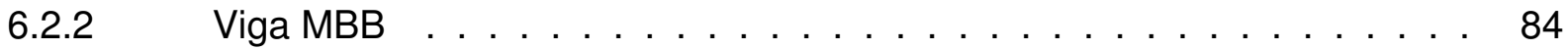

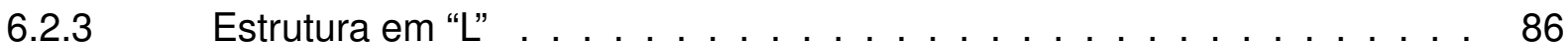

6.2.4 Convergências das funções objetivo . . . . . . . . . . . . . . . . 91

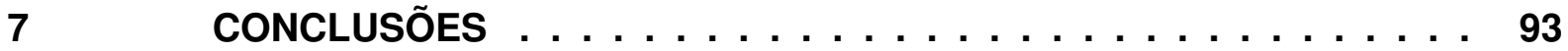

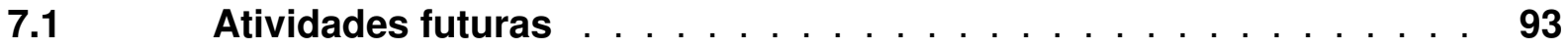

REFERÊNCIAS . . . . . . . . . . . . . . . . . . 95

APÊNDICE A - IMPLEMENTAÇÃO DO Método dos Elementos Finitos (MEF) PARA PROBLEMAS ESTRUTURAIS . . . . . . 103 APÊNDICE B - IMPLEMENTAÇÃO DO FILTRO DE HELMHOLTZ . . . 105 


\section{INTRODUÇÃO}

\subsection{Compósitos reforçados com fibra}

Materiais compósitos são compostos de dois ou mais componentes combinados em um nível macroscópico, cujo o desempenho mecânico é projetado para ser superior aos componentes individuais atuando independentemente. Compósitos Reforçados com Fibras (CRF) são um importante grupo entre os materiais compósitos. Estes materiais são constituídos de fibras com boas propriedades mecânicas inseridas em uma matriz (ver Fig. 1.1) (DANIEL et al., 1994; KAW, 2005; VASILIEV; MOROZOV, 2001).

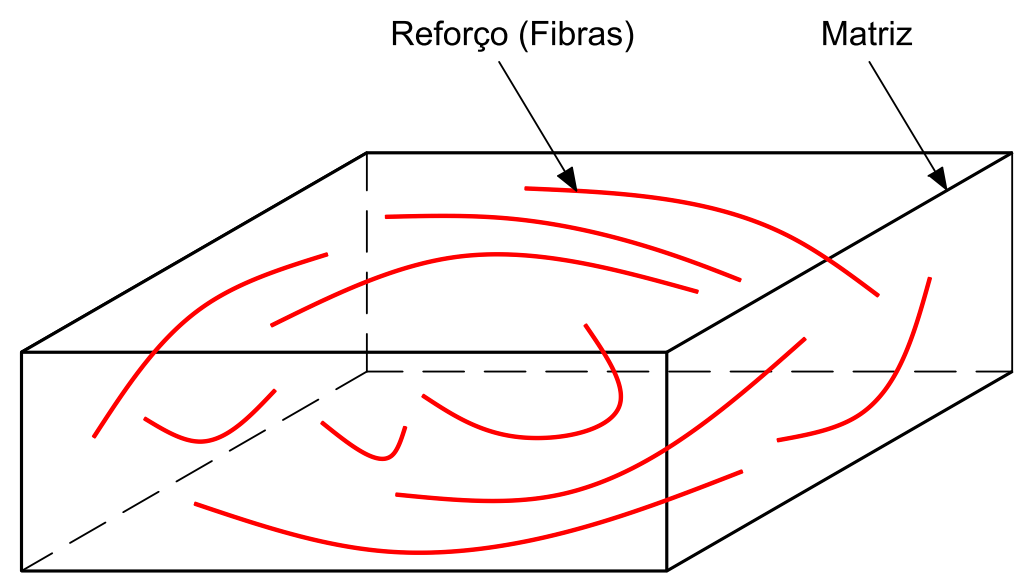

Figura 1.1 - Representação genérica de um compósito reforçado com fibras.

Apesar de evidências comprovarem a utilização de fibras como material de reforço em 1500 A.C, CRF modernos surgiram em meados do século XX, e desde então, vem sendo empregados progressivamente em diversas aplicações (KAW, 2005; VASILIEV; MOROZOV, 2001).

Em função da ampla aplicabilidade destes materiais em indústrias aeronáuticas , automobilísticas, navais, esportivas, de construção civil e em áreas como biomedicina, novas tecnologias de manufatura aditiva para CRF estão sendo desenvolvidas, como pode ser visto nos trabalhos de Ning et al. (2015), Ning et al. (2017), Quan et al. (2015), Hou et al. (2018). Um exemplo dessas novas tecnologias pode ser visto na Fig. 1.2, que apresenta um braço robótico utilizado pela National Aeronautics and Space Administration (NASA) para fabricar, com baixo custo e alta velocidade, estruturas compósitas utilizadas em foguetes. 
Com este equipamento é possível fabricar peças com mais de oito metros de diâmetro, utilizando uma tecnologia que permite o posicionamento das fibras de forma automática.

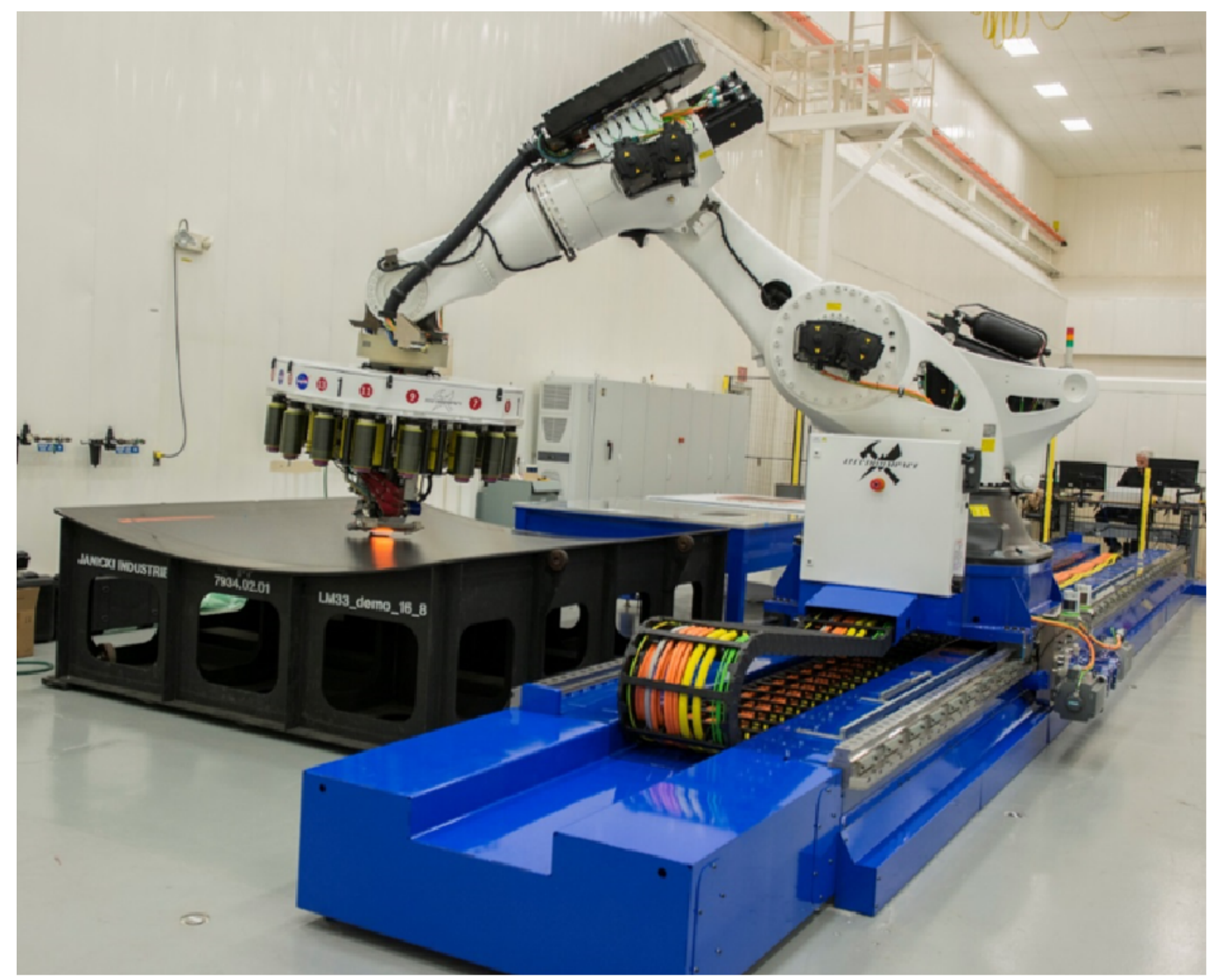

Figura 1.2 - Braço robótico para manufatura aditiva de materiais reforçados com fibra de carbono. Retirado de: https: //www.nasa.gov/ centers/ marshall/ news/ news/releases/2015/ robotic - manufacturing - system - will - build - biggest composite - rocket - parts - ever - made.html

Uma outra abordagem de inserção de fibras está sendo estudada na área de construção civil, onde desenvolve-se equipamentos de manufatura aditiva para concreto que automaticamente inserem fibras, que podem ser de carbono, vidro ou basalto por exemplo, para atuar como reforço na estrutura (HAMBACH; RUTZEN; VOLKMER, 2019). Este tipo de tecnologia vem sido desenvolvida para ajudar a mitigar o impacto ambiental provocado pela fabricação e utilização de cimento, principal componente do concreto. Outra importante justificativa, é a economia de energia, tanto na produção do cimento quanto na produção do aço utilizado em armaduras convencionais. A Fig. 1.3 representa o bico de impressão de um equipamento de manufatura aditiva onde as fibras são inseridas durante o processo de deposição de concreto.

O posicionamento das fibras em um CRF altera as propriedades destes materiais, 


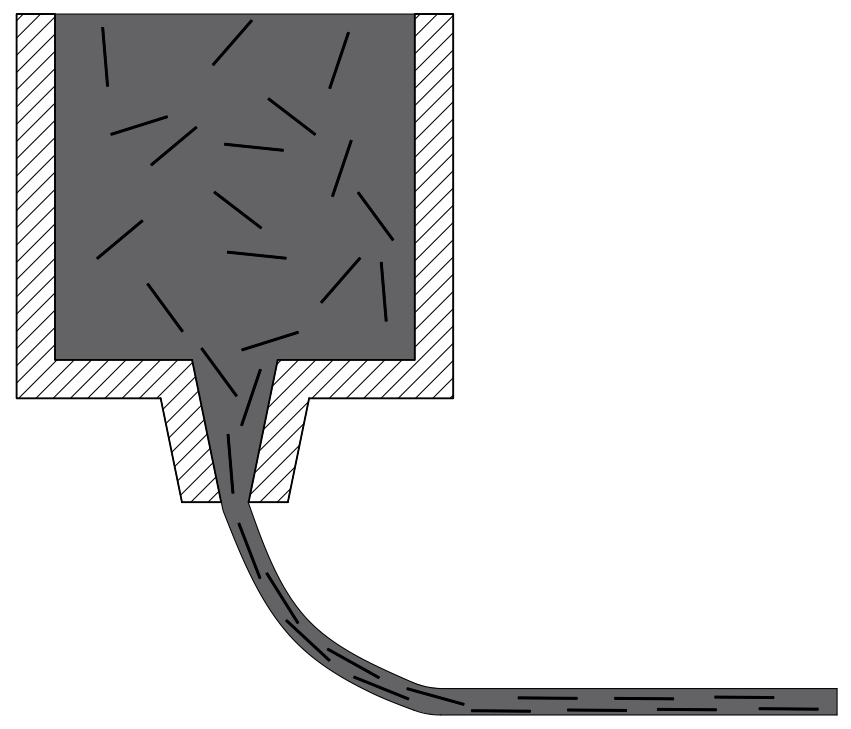

Figura 1.3 - Representação do bico de impressão de um equipamento de manufatura aditiva para concreto reforçado com fibras. Adaptado de (HAMBACH; RUTZEN; VOLKMER, 2019)

portanto, utilizando tecnologias que permitem que este posicionamento seja personalizado, é possível obter estruturas com propriedades otimizadas para determinadas aplicações. Em função disto, há um grande número de trabalhos baseados em otimização do posicionamento das fibras em CRF publicados, onde diferentes abordagens são utilizadas. Exemplos que mostram a diversidade destes métodos pode ser encontrados em Venkataraman e Haftka (1999), Awad et al. (2012), Nikbakt, Kamarian e Shakeri (2018). Um desses métodos é o algoritmo Genético, utilizado em grande parte destes trabalhos. Algoritmos genéticos são utilizados para aprimorar o comportamento aeroelástico (DILLINGER et al., 2013), aprimorar a resistência ao choque (KIM; KIM; KIM, 2015), aprimorar o desempenho de controles ativos (MOITA et al., 2006; MOITA et al., 2008) aprimorar propriedades de resistência a torção (HERATH et al., 2017), aprimorar o comportamento vibracional (SUDHAGAR et al., 2017), maximização da carga de flambagem (QIU; ZHU, 2014; DEVECI; AYDIN; ARTEM, 2016), minimização de respostas dinâmicas (CHO, 2009; AZARAFZA et al., 2009), maximização da rigidez (MEJLEJ et al., 2017), maximização do fator de segurança (NICHOLAS; PADMANABAN; SOFIA, 2012) e minimização da tensão mecânica(JAFARI; ROHANI, 2016). Um dos principais argumentos para a utilização desta abordagem é o fato de ser possível se obter uma convergência global e não um mínimo local. No entanto, isso não ocorre para problemas com muitas variáveis de projeto. Além disso, com o aumento do número de variáveis de projeto, o número de combinações possíveis para a solução do problema aumenta exponencialmente. Outras desvantagens da utilização destes algoritimos são enumeradas em Sigmund (2011). 
De acordo com Sigmund (2011), Métodos Baseados em Gradiente (MBG) são mais eficientes quando comparados a algoritmos genéticos. Na literatura existem trabalhos que utilizam MBG para determinar a orientação otimizada das fibras. Butyrin et al. (2009) utilizam um MBG para solucionar um problema de otimização aplicado a estruturas compósitas em que a variável de projeto é a espessura. A otimização é aplicada a vigas, placas e painéis com orifícios, sujeitos a momentos e cargas de flexão, sendo o objetivo deste trabalho a minimização da massa sujeita a critérios de fratura. O problema de minimização de massa também é explorado por António, Barbosa e Dinis (2000), onde o método dos gradientes conjugados é utilizado considerando como restrições a deflexão e a tensão mecânica e por (SØRENSEN; LUND, 2015), que utilizando restrições de fundição como filtro de controle, apresentam um MBG para otimizar a espessura de CRF. (IKEYA; SHIMODA; SHI, 2016) solucionam o problema de maximização da rigidez utilizando um método denominado método do gradiente $H^{1}$ aplicado em duas etapas. Em uma primeira etapa uma otimização de forma é realizada e em seguida a espessura do laminado é otimizada.

Independentemente do tipo de algoritmo, para a maioria dos casos, considerar o ângulo da fibra diretamente como uma variável de projeto no problema de otimização pode incorrer em problemas de múltiplos mínimos locais, em função deste tipo de formulação tornar o espaço de solução não convexo, o que foi detalhado no trabalho de Stegmann e Lund (2005). Para contornar estes problemas Stegmann e Lund (2005) propõem um método, denominado Otimização Discreta de Material (ODM), onde ângulos candidatos são escolhidos a priori. O método consiste em utilizar uma soma ponderada dos tensores constitutivos correspondentes a cada ângulo candidato e com isso definir um tensor constitutivo efetivo. Uma desvantagem do ODM é o fato de o número de variáveis de projeto por elemento aumentarem linearmente com o aumento do número de ângulos candidatos.

Bruyneel (2011) propõe um método denominado Parametrização por Funções de Forma (PFF) onde a ideia da utilização de ângulos candidatos também é utilizada. No método PFF, as funções de ponderação são funções de forma do MEF e apenas duas variáveis de projeto por elemento são necessárias. Este método é formulado para que quatro ângulos candidatos sejam utilizados e sua implementação é menos complexa quando comparada ao método ODM. Bruyneel (2011) utiliza como candidatos os ângulos $0^{\circ}, \pm 45^{\circ}$ e $90^{\circ}$, os quais são comumente utilizados em aplicações aeronáuticas (KIYONO; SILVA; REDDY, 2017). Uma generalização do método PFF onde não há limites de escolhas de ângulos candidatos é proposta por Gao, Zhang e Duysinx (2012). Neste método, denominado Parametrização por Codificação Binária (PCB), o número de variáveis de projeto por elemento diminui logaritmicamente com o aumento do número de ângulos candidatos. Um método denominado Otimização de Fibra por Distribuição Normal (OFDN), que utiliza apenas uma variável por elemento, foi proposto por Kiyono, Silva e Reddy (2017). 
O OFDN utiliza a mesma normalização para as funções de ponderação do método ODM, porém, os pesos são determinados por meio de uma função de distribuição normal, utilizada para interpolação no trabalho de Yin e Ananthasuresh (2001), onde foi explorada para otimização com multi-materiais.

Métodos que utilizam ângulos candidatos são uma boa estratégia para contornar os problemas de mínimo local. Existe, no entanto, um método que considera o ângulo da fibra diretamente como uma variável de projeto e que não apresenta este tipo de problema. $O$ Modelo de Interpolação Autopenalizável da Orientação da Fibra (MIAFIB) (ver Seção 3.5.2), proposto por Salas et al. (2018), que tem como principal característica a definição das funções seno e cosseno por meio de séries de Taylor, consegue contornar o problema de mínimos locais por meio de um método de continuação que ocorre com o aumento do número de termos nas séries de Taylor durante o processo de otimização, por esta razão, o modelo é chamado auto-penalizável. O MIAFIB, assim como o OFDN, utiliza apenas uma variável de projeto por elemento.

\subsection{Procedimento de projeto de CRF utilizando OT}

A OT, em sua abordagem tradicional, consiste em combinar métodos numéricos, como o MEF, com algoritmos de otimização para determinar a distribuição otimizada de um determinado material em um domínio fixo. Uma das principais características da OT é o fato de o domínio permanecer o mesmo durante todo o processo de otimização. A topologia final é definida pela forma como uma variável de projeto é distribuída neste domínio.

A Fig. 1.4 apresenta o procedimento típico para o projeto de CRF utilizando OT. No início, define-se um domínio e suas respectivas condições de contorno. Após isto, o domínio é discretizado. Define-se a função (ou funções) objetivo, que pode ser, por exemplo, a rigidez da estrutura. Restrições, como a de volume, podem ser impostas. Uma distribuição inicial com os valores das variáveis de projeto é fornecida para o algoritmo de OT. Com essa informação, o problema de MEF é solucionado para a determinação dos deslocamentos. $O$ valor da função objetivo é determinado, os gradientes da função objetivo e das restrições são calculados e as restrições são verificadas. Um algoritmo de otimização utiliza essas informações para determinar uma nova configuração para as variáveis de projeto no domínio e um critério de parada é verificado. Este processo é repetido até que o critério de parada, como um critério de convergência ou número máximo de iterações, seja alcançado. As fronteiras da topologia obtida dependem da discretização utilizada, e em função disto, o resultado da otimização topológica deve passar por um pós-processamento. Antes da fabricação, o resultado do pós-processamento é verificado por meio de simulação numérica. 


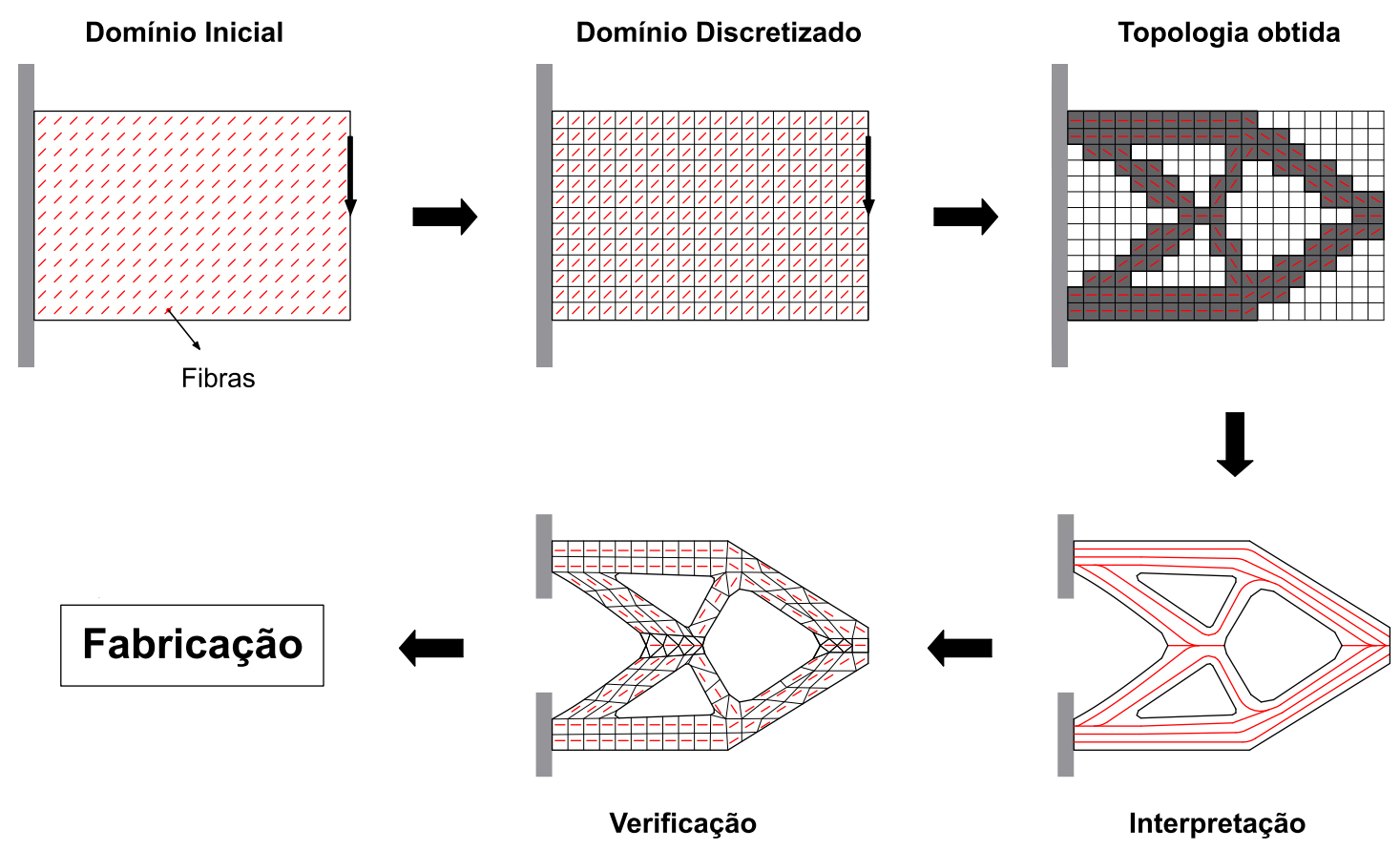

Figura 1.4 - Procedimento de OT aplicado ao projeto de CRF

\subsection{Objetivos}

Este trabalho tem como objetivo principal o desenvolvimento de um algoritmo baseado em OT para o projeto de estruturas compósitas reforçadas com fibras, passíveis de ser fabricadas com tecnologia de manufatura aditiva. Propõe-se primeiramente determinar apenas a orientação otimizada das fibras e após isto, determinar simultaneamente a orientação das fibras e a distribuição otimizada de material. O projeto consiste em obter a máxima rigidez da estrutura, que para o caso onde a distribuição de material também é considerada, está sujeita a uma restrição de volume. Para isto, são utilizadas formulações baseadas nos trabalhos de Bendsøe (1989), Rozvany, Zhou e Birker (1992), Kiyono, Silva e Reddy (2017) e Salas et al. (2018). Para reduzir o problema de descontinuidade das fibras e tratar problemas característicos da OT é utilizado um filtro proposto por Lazarov e Sigmund (2011), baseado em uma equação modificada de Helmholtz. Uma restrição de continuidade baseada no modelo de Borrvall é utilizada para garantir a continuidade e mitigar o problema de acumulo de fibras. A implementação é realizada utilizando-se o ambiente FEniCS, que possui código aberto, combinado com o pacote Dolfin-Adjoint. O algoritmo de otimização utilizado para atualizar a distribuição das variáveis de projeto é o método do ponto interior implementado no pacote IPOpt. 


\subsection{Motivação}

Materiais compósitos tem sido amplamente utilizados em diversas áreas da engenharia e em função disto, novas tecnologias de manufatura aditiva dedicadas a este tipo de material estão sendo desenvolvidas. O fato destas novas tecnologias possibilitarem o posicionamento das fibras de modo personalizado, permite que estruturas otimizadas para determinadas aplicações sejam construídas. Porém, definir a configuração otimizada de um CRF é uma tarefa complexa. O desenvolvimento de um algoritmo que realiza este processo de forma automatizada, considerando a importante questão da continuidade das fibras, que quando não obtida pode resultar em concentração de tensão e dificuldades no processo de manufatura, facilitará o projeto de CRF específicos para cada aplicação. 


\section{MODELAGEM DE COMPÓSITOS REFORÇADOS COM FIBRAS}

O Compósito Reforçado com Fibras CRF é um material anisotrópico, porém, seu comportamento pode ser aproximado considerando-o um material ortotrópico, onde há três planos de simetria mutuamente perpendiculares, ou como um material transversalmente isotrópico, um tipo especial de material ortotrópico em que um de seus planos principais é um plano de isotropia e perpendicular a este plano, há a direção principal da fibra (BONET; BURTON, 1998; DANIEL et al., 1994; KAW, 2005).

\subsection{Materiais ortotrópicos}

A equação constitutiva para um material genérico deve considerar uma relação entre tensão e deformação não-linear elástica e um comportamento totalmente anisotrópico. Para o caso de um material CRF é aceitável assumir um comportamento linear elástico, porém, assumir que o CRF tem um comportamento isotrópico é inaceitável para a maioria dos casos (KAW, 2005). Portanto, a equação constitutiva para CRF pode ser definida por meio da Eq. (2.1) (DANIEL et al., 1994; KAW, 2005)

$$
\left[\begin{array}{l}
\sigma_{11} \\
\sigma_{22} \\
\sigma_{33} \\
\sigma_{23} \\
\sigma_{31} \\
\sigma_{12} \\
\sigma_{32} \\
\sigma_{13} \\
\sigma_{21}
\end{array}\right]=\left[\begin{array}{lllllllll}
C_{1111} & C_{1122} & C_{1133} & C_{1123} & C_{1131} & C_{1112} & C_{1132} & C_{1113} & C_{1121} \\
C_{2211} & C_{2222} & C_{2233} & C_{2223} & C_{2231} & C_{2212} & C_{2232} & C_{2213} & C_{221} \\
C_{3311} & C_{3322} & C_{3333} & C_{3323} & C_{3331} & C_{3312} & C_{3332} & C_{3313} & C_{3321} \\
C_{2311} & C_{2322} & C_{2333} & C_{2323} & C_{2331} & C_{2312} & C_{2332} & C_{2313} & C_{2321} \\
C_{3111} & C_{3122} & C_{3133} & C_{3123} & C_{3131} & C_{3112} & C_{3132} & C_{3113} & C_{3121} \\
C_{1211} & C_{1222} & C_{1233} & C_{1223} & C_{1231} & C_{1212} & C_{1232} & C_{1213} & C_{1221} \\
C_{3211} & C_{3222} & C_{3233} & C_{3223} & C_{3231} & C_{3212} & C_{3232} & C_{3213} & C_{3221} \\
C_{1311} & C_{1322} & C_{1333} & C_{1323} & C_{1331} & C_{1312} & C_{1332} & C_{1313} & C_{1321} \\
C_{2111} & C_{2122} & C_{2133} & C_{2123} & C_{2131} & C_{2112} & C_{2132} & C_{2113} & C_{2121}
\end{array}\right]\left[\begin{array}{l}
\varepsilon_{11} \\
\varepsilon_{22} \\
\varepsilon_{33} \\
\varepsilon_{23} \\
\varepsilon_{31} \\
\varepsilon_{12} \\
\varepsilon_{32} \\
\varepsilon_{13} \\
\varepsilon_{21}
\end{array}\right]
$$

ou em notação indicial

$$
\sigma_{i j}=C_{i j k l} \varepsilon_{k l}
$$

onde $\sigma_{i j}$ são os componente do tensor de tensões, $C_{i j k l}$ são os componentes do tensor constitutivo e $\varepsilon_{k l}$ são os componentes do tensor de deformações. Portanto, são necessárias 
81 constantes para caracterizar completamente um material genérico. No entanto, ao se considerar a simetria dos tensores de tensão e deformação (ver Eq. (2.3)), reduz-se o número de constantes elásticas para 36 (DANIEL et al., 1994).

$$
\begin{aligned}
\sigma_{i j} & =\sigma_{j i} \\
\varepsilon_{i j} & =\varepsilon_{j i}
\end{aligned} \quad(i, j, k, l=1,2,3)
$$

com isso, é possível utilizar a notação de Voigt para escrever a equação constitutiva para um sólido anisotrópico de forma compactada (ver Eq. (2.4)) (DANIEL et al., 1994; KAW, 2005)

$$
\left[\begin{array}{l}
\sigma_{1} \\
\sigma_{2} \\
\sigma_{3} \\
\tau_{23} \\
\tau_{31} \\
\tau_{12}
\end{array}\right]=\left[\begin{array}{llllll}
C_{11} & C_{12} & C_{13} & C_{14} & C_{15} & C_{16} \\
C_{21} & C_{22} & C_{23} & C_{24} & C_{25} & C_{26} \\
C_{31} & C_{32} & C_{33} & C_{34} & C_{35} & C_{36} \\
C_{41} & C_{42} & C_{43} & C_{44} & C_{45} & C_{46} \\
C_{51} & C_{52} & C_{53} & C_{54} & C_{55} & C_{56} \\
C_{61} & C_{62} & C_{63} & C_{64} & C_{65} & C_{66}
\end{array}\right]\left[\begin{array}{c}
\varepsilon_{1} \\
\varepsilon_{2} \\
\varepsilon_{3} \\
\gamma_{23} \\
\gamma_{31} \\
\gamma_{12}
\end{array}\right]
$$

ou em notação indicial

$$
\sigma_{i}=C_{i j} \varepsilon_{j} \quad(i, j=1,2,3 \ldots, 6)
$$

A relação entre tensão e deformação pode ser estabelecida convenientemente por meio de um tensor de flexibilidade $\mathbf{S}$ (ver Eq. (2.6))

$$
\left[\begin{array}{c}
\varepsilon_{1} \\
\varepsilon_{2} \\
\varepsilon_{3} \\
\gamma_{23} \\
\gamma_{31} \\
\gamma_{12}
\end{array}\right]=\left[\begin{array}{llllll}
S_{11} & S_{12} & S_{13} & S_{14} & S_{15} & S_{16} \\
S_{21} & S_{22} & S_{23} & S_{24} & S_{25} & S_{26} \\
S_{31} & S_{32} & S_{33} & S_{34} & S_{35} & S_{36} \\
S_{41} & S_{42} & S_{43} & S_{44} & S_{45} & S_{46} \\
S_{51} & S_{52} & S_{53} & S_{54} & S_{55} & S_{56} \\
S_{61} & S_{62} & S_{63} & S_{64} & S_{65} & S_{66}
\end{array}\right]\left[\begin{array}{c}
\sigma_{1} \\
\sigma_{2} \\
\sigma_{3} \\
\tau_{23} \\
\tau_{31} \\
\tau_{12}
\end{array}\right]
$$

Admitindo que o material é hiperelástico, há um potencial escalar $\Psi(\varepsilon)$ denominado Energia de Deformação Específica que pode ser expresso por meio da Eq. (2.7) (PIMENTA, 2008; LAl et al., 2009; KAW, 2005; DANIEL et al., 1994)

$$
\Psi=\frac{1}{2} C_{i j} \varepsilon_{i} \varepsilon_{j}
$$

Diferenciando-se a Eq. (2.7), é possível obter a relação de tensão-deformação (ver Eqs. (2.5) 
e (2.8) (DANIEL et al., 1994; KAW, 2005; LAl et al., 2009)

$$
\frac{\partial \Psi}{\partial \varepsilon_{i}}=C_{i j} \varepsilon_{j}
$$

diferenciando novamente obtêm-se (KAW, 2005; DANIEL et al., 1994)

$$
C_{i j}=\frac{\partial^{2} \Psi}{\partial \varepsilon_{i} \partial \varepsilon_{j}}
$$

Se $\frac{\partial^{2} \Psi}{\partial \varepsilon_{i} \partial \varepsilon_{j}}$ e $\frac{\partial^{2} \Psi}{\partial \varepsilon_{j} \partial \varepsilon_{i}}$ existem e são contínuas, a ordem de diferenciação da Eq. (2.9) não interfere no resultado (teorema de Shuwarz (TRAHAN, 1969)). Portanto, assumindo esta hipótese, pode-se dizer que C é simétrico (ver Eq. (2.10)) e isto implica que vinte e uma constantes elásticas são suficientes para caracterizá-lo. De maneira similar, pode-se verificar que $\mathbf{S}$ também é simétrico (ver Eq. (2.11)) (PIMENTA, 2008; LAl et al., 2009; DANIEL et al., 1994; KAW, 2005).

$$
\begin{gathered}
C_{i j}=C_{j i} \\
S_{i j}=S_{j i}
\end{gathered}
$$

CRF são materiais que podem ser caracterizados como ortotrópicos, ou seja, existem no CRF três planos de simetria material mutuamente perpendiculares (ver Fig. 2.1). Isto faz com que alguns componentes do tensor constitutivo $\mathbf{C}$ e do tensor de flexibilidade $\mathbf{S}$ sejam inter-relacionados, tornando possível reduzir o número de constantes elásticas independentes para nove (ver Eq. (2.12)) (DANIEL et al., 1994; KAW, 2005).

$$
\begin{aligned}
& {\left[\begin{array}{l}
\sigma_{1} \\
\sigma_{2} \\
\sigma_{3} \\
\tau_{23} \\
\tau_{31} \\
\tau_{12}
\end{array}\right]=\left[\begin{array}{cccccc}
C_{11} & C_{12} & C_{13} & 0 & 0 & 0 \\
C_{12} & C_{22} & C_{23} & 0 & 0 & 0 \\
C_{13} & C_{23} & C_{33} & 0 & 0 & 0 \\
0 & 0 & 0 & C_{44} & 0 & 0 \\
0 & 0 & 0 & 0 & C_{55} & 0 \\
0 & 0 & 0 & 0 & 0 & C_{66}
\end{array}\right]\left[\begin{array}{c}
\varepsilon_{1} \\
\varepsilon_{2} \\
\varepsilon_{3} \\
\gamma_{23} \\
\gamma_{31} \\
\gamma_{12}
\end{array}\right]} \\
& {\left[\begin{array}{l}
\varepsilon_{1} \\
\varepsilon_{2} \\
\varepsilon_{3} \\
\gamma_{23} \\
\gamma_{31} \\
\gamma_{12}
\end{array}\right]=\left[\begin{array}{cccccc}
S_{11} & S_{12} & S_{13} & 0 & 0 & 0 \\
S_{12} & S_{22} & S_{23} & 0 & 0 & 0 \\
S_{13} & S_{23} & S_{33} & 0 & 0 & 0 \\
0 & 0 & 0 & S_{44} & 0 & 0 \\
0 & 0 & 0 & 0 & S_{55} & 0 \\
0 & 0 & 0 & 0 & 0 & S_{66}
\end{array}\right]\left[\begin{array}{l}
\sigma_{1} \\
\sigma_{2} \\
\sigma_{3} \\
\tau_{23} \\
\tau_{31} \\
\tau_{12}
\end{array}\right]}
\end{aligned}
$$




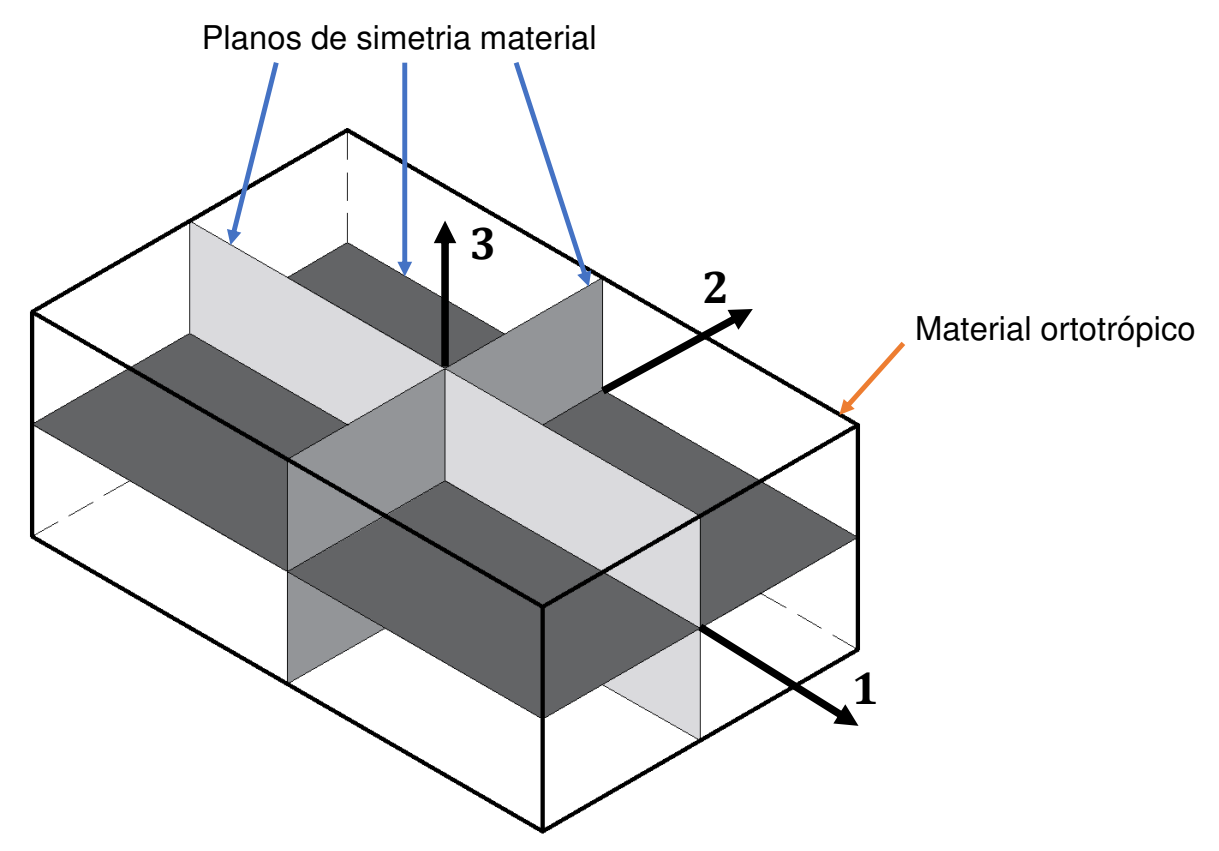

Figura 2.1 - Planos de simetria material em um material ortotrópico

Os componentes $S_{i j}$ e $C_{i j}$ são determinados em função de três módulos de Young $E_{i}, \quad(i=1,2,3$, onde $i$ representa os eixos materiais), seis coeficientes de Poisson $\left(v_{12}, v_{13}, v_{21}, v_{23}, v_{31}\right.$ e $v_{32}$, dois para cada plano) e três módulos de cisalhamento $\left(G_{23}, G_{31} \mathrm{e}\right.$ $G_{12}$, um para cada plano). Os coeficientes não são independentes entre si, o que implica que equações de reciprocidade devem ser respeitadas (ver Eq. (2.14)) (KAW, 2005). As relações estabelecidas na Eq. (2.14) fazem com que existam nove constantes independentes nos tensores constitutivos $\mathbf{C}$ e flexibilidade $\mathbf{S}$

$$
\frac{v_{i j}}{E_{i}}=\frac{v_{j i}}{E_{j}} \quad \text { para } i \neq j \text { e } i, j=1,2,3
$$

O tensor de flexibilidade $\mathbf{S}$ pode, então, ser reescrito em função das constantes materiais (ver Eq. (2.15)) e o tensor constitutivo $\mathbf{C}$ pode ser obtido invertendo-se o tensor $\mathbf{S}$ (ver Eq. (2.16)) (KAW, 2005)

$$
\mathbf{S}=\left[\begin{array}{cccccc}
\frac{1}{E_{1}} & -\frac{v_{12}}{E_{1}} & -\frac{v_{13}}{E_{1}} & 0 & 0 & 0 \\
-\frac{v_{21}}{E_{2}} & -\frac{1}{E_{2}} & -\frac{v_{23}}{E_{2}} & 0 & 0 & 0 \\
-\frac{v_{31}}{E_{3}} & -\frac{v_{32}}{E_{3}} & -\frac{1}{E_{3}} & 0 & 0 & 0 \\
0 & 0 & 0 & \frac{1}{G_{23}} & 0 & 0 \\
0 & 0 & 0 & 0 & \frac{1}{G_{31}} & 0 \\
0 & 0 & 0 & 0 & 0 & \frac{1}{G_{12}}
\end{array}\right]
$$




$$
\mathbf{C}=\left[\begin{array}{cccccc}
\frac{1-v_{23} v_{32}}{E_{2} E_{3} \Delta} & \frac{v_{21}+v_{23} v_{31}}{E_{2} E_{3} \Delta} & \frac{v_{31}+v_{21} v_{32}}{E_{2} E_{3} \Delta} & 0 & 0 & 0 \\
\frac{v_{21}+v_{2} v_{31}}{E_{2} E_{3} \Delta} & \frac{1-v_{13} v_{31}}{E_{1} E_{3} \Delta} & \frac{v_{32}+v_{12} v_{31}}{E_{1} E_{3} \Delta} & 0 & 0 & 0 \\
\frac{v_{31}+v_{21} v_{32}}{E_{2} E_{3} \Delta} & \frac{v_{32}+v_{12} v_{31}}{E_{1} E_{3} \Delta} & -\frac{1-v_{12} v_{21}}{E_{1} E_{2} \Delta} & 0 & 0 & 0 \\
0 & 0 & 0 & G_{23} & 0 & 0 \\
0 & 0 & 0 & 0 & G_{31} & 0 \\
0 & 0 & 0 & 0 & 0 & G_{12}
\end{array}\right]
$$

onde o termo $\Delta$ (ver Eq. (2.17)) é utilizado para facilitar a notação.

$$
\Delta=\frac{1-v_{12} v_{21}-v_{23} v_{32}-v_{13} v_{31}-2 v_{21} v_{32} v_{13}}{E_{1} E_{2} E_{3}}
$$

\subsection{Estado plano de tensão para Compósitos Reforçados com Fibras}

Para placas de pequena espessura sem carregamentos fora de seu plano, a hipótese de Estado Plano de Tensão (EPT) pode ser assumida. Com isso, as relações de tensão e deformação podem ser reescritas (ver Eqs. (2.18) e (2.19)) e os tensores de flexibilidade $\mathbf{S}$ e constitutivo $\mathbf{Q}$ para o EPT passam a ter quatro componentes independentes cada (KAW, 2005; DANIEL et al., 1994).

$$
\begin{aligned}
& {\left[\begin{array}{l}
\varepsilon_{1} \\
\varepsilon_{2} \\
\varepsilon_{3}
\end{array}\right]=\left[\begin{array}{ccc}
S_{11} & S_{12} & 0 \\
S_{12} & S_{22} & 0 \\
0 & 0 & S_{66}
\end{array}\right]\left[\begin{array}{l}
\sigma_{1} \\
\sigma_{2} \\
\tau_{12}
\end{array}\right]} \\
& {\left[\begin{array}{l}
\sigma_{1} \\
\sigma_{2} \\
\tau_{12}
\end{array}\right]=\left[\begin{array}{ccc}
Q_{11} & Q_{12} & 0 \\
Q_{12} & Q_{22} & 0 \\
0 & 0 & Q_{66}
\end{array}\right]\left[\begin{array}{l}
\varepsilon_{1} \\
\varepsilon_{2} \\
\gamma_{12}
\end{array}\right]}
\end{aligned}
$$

onde os componentes $Q_{i j}$ podem ser determinados em função dos componentes $S_{i j}$ (ver Eq. (2.20)) (KAW, 2005).

$$
\begin{gathered}
Q_{11}=\frac{S_{22}}{S_{11} S_{22}-S_{12}^{2}} \\
Q_{12}=-\frac{S_{12}}{S_{11} S_{22}-S_{12}^{2}} \\
Q_{22}=-\frac{S_{11}}{S_{11} S_{22}-S_{12}^{2}}
\end{gathered}
$$




$$
Q_{66}=\frac{1}{S_{66}}
$$

$S_{i j}$ para o EPT podem ser obtidos utilizando-se o módulo de Young, o coeficiente de Poisson e o módulo de cisalhamento (ver Eq. (2.21)) (KAW, 2005)

$$
\mathbf{S}=\left[\begin{array}{ccc}
\frac{1}{E_{1}} & -\frac{\nu_{12}}{E_{1}} & 0 \\
-\frac{\nu_{12}}{E_{1}} & \frac{1}{E_{2}} & 0 \\
0 & 0 & -\frac{1}{G_{12}}
\end{array}\right]
$$

\subsection{Mudança de coordenadas}

A equação constitutiva para um CRF deve levar em consideração o ângulo da fibra, o que pode ser feito por meio de uma mudança de coordenadas. O sistema de coordenadas utilizado para considerar o ângulo da fibra é mostrado na Fig. 2.2, onde os eixos $x, y, z$ representam coordenadas globais e os eixos 1,2,3 representam coordenadas locais.

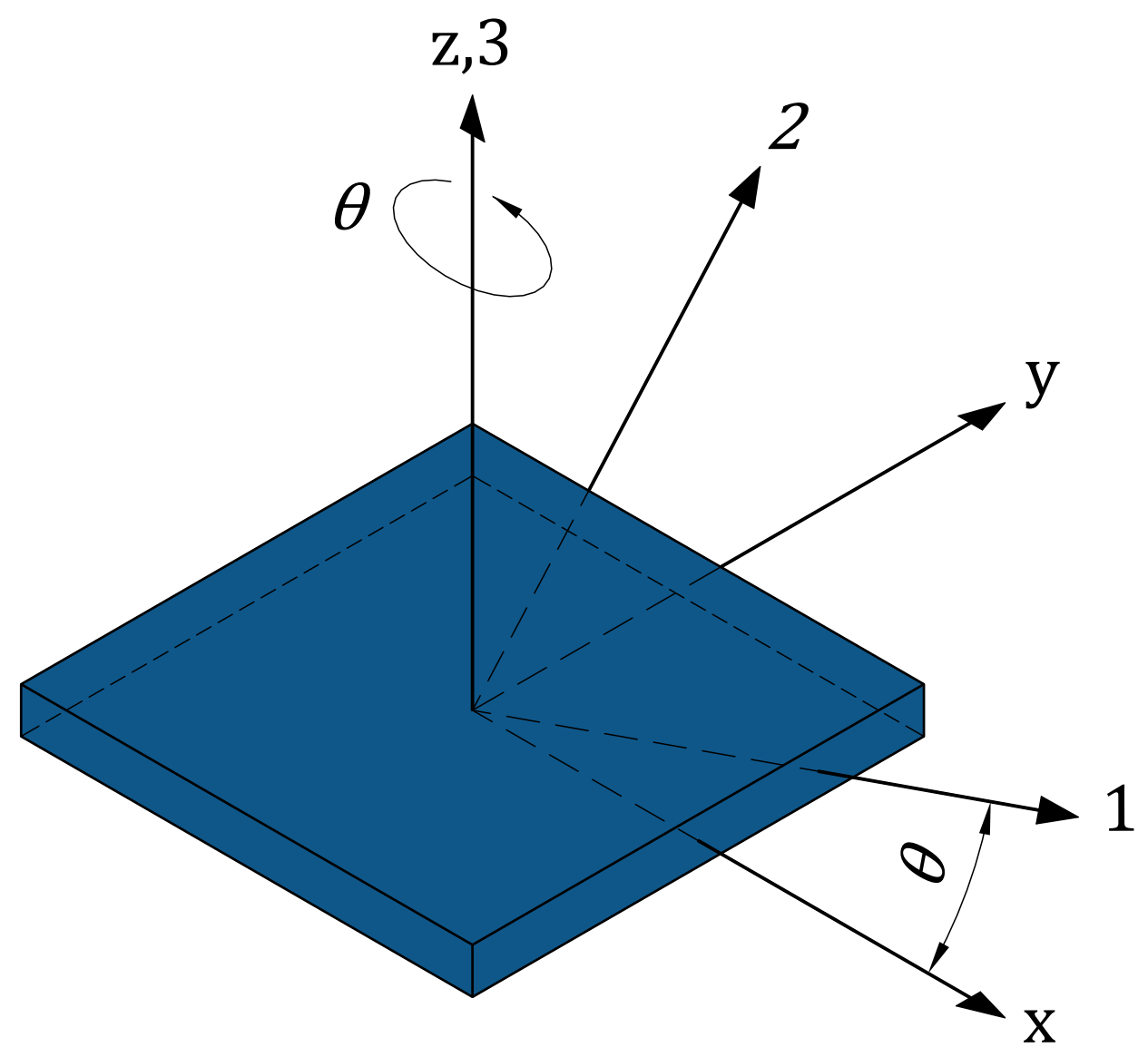

Figura 2.2 - Eixos globais e locais em um CRF 
O ângulo entre os dois sistemas é denotado por $\theta$ e gira em torno do eixo $z$. A mudança de coordenadas das tensões do sistema 1,2,3 para o sistema $x, y, z$ é dada pela Eq. (2.22)

$$
\left[\begin{array}{c}
\sigma_{x} \\
\sigma_{y} \\
\sigma_{z} \\
\tau_{y z} \\
\tau_{z x} \\
\tau_{x y}
\end{array}\right]=\mathbf{T}^{-1}\left[\begin{array}{c}
\sigma_{1} \\
\sigma_{2} \\
\sigma_{3} \\
\tau_{23} \\
\tau_{31} \\
\tau_{12}
\end{array}\right]
$$

onde a matriz de transformação $\mathbf{T}$ é definida por senos e cossenos (ver Eq. (2.23)) (ALMEIDA, 2018)

$$
\mathbf{T}=\left[\begin{array}{cccccc}
c^{2} & s^{2} & 0 & 0 & 0 & 2 c s \\
s^{2} & c^{2} & 0 & 0 & 0 & -2 c s \\
0 & 0 & 1 & 0 & 0 & 0 \\
0 & 0 & 0 & c & -s & 0 \\
0 & 0 & 0 & s & c & 0 \\
-c s & c s & 0 & 0 & 0 & c^{2}-s^{2}
\end{array}\right], \quad c=\cos (\theta), \quad s=\operatorname{sen}(\theta)
$$

A mudança de coordenadas das deformações dos sistema $x, y, z$ para o sistema 1,2, 3 é definida pela equação Eq. (2.24) (KAW, 2005; ALMEIDA, 2018)

$$
\left[\begin{array}{c}
\varepsilon_{1} \\
\varepsilon_{2} \\
\varepsilon_{3} \\
\gamma_{23} \\
\gamma_{31} \\
\gamma_{12}
\end{array}\right]=\mathbf{R} \mathbf{T} \mathbf{R}^{-1}\left[\begin{array}{c}
\varepsilon_{x} \\
\varepsilon_{y} \\
\varepsilon_{z} \\
\gamma_{y z} \\
\gamma_{z x} \\
\gamma_{x y}
\end{array}\right]
$$

onde $\mathbf{R}$ é a matriz de Reuter (ver Eq. (2.25)) (ALMEIDA, 2018)

$$
\mathbf{R}=\left[\begin{array}{llllll}
1 & 0 & 0 & 0 & 0 & 0 \\
0 & 1 & 0 & 0 & 0 & 0 \\
0 & 0 & 1 & 0 & 0 & 0 \\
0 & 0 & 0 & 2 & 0 & 0 \\
0 & 0 & 0 & 0 & 2 & 0 \\
0 & 0 & 0 & 0 & 0 & 2
\end{array}\right]
$$

A partir das Eqs. (2.12), (2.22) e (2.24) pode-se definir a relação entre tensão e 
deformação em coordenadas globais (ver Eq. (2.26)) (ALMEIDA, 2018; KAW, 2005)

$$
\left[\begin{array}{c}
\sigma_{x} \\
\sigma_{y} \\
\sigma_{z} \\
\tau_{y z} \\
\tau_{z x} \\
\tau_{x y}
\end{array}\right]=\mathbf{T}^{-1} \mathbf{C} \mathbf{R} \mathbf{T} \mathbf{R}^{-1}\left[\begin{array}{c}
\varepsilon_{x} \\
\varepsilon_{y} \\
\varepsilon_{z} \\
\gamma_{y z} \\
\gamma_{z x} \\
\gamma_{x y}
\end{array}\right]=\overline{\mathbf{C}}\left[\begin{array}{c}
\varepsilon_{x} \\
\varepsilon_{y} \\
\varepsilon_{z} \\
\gamma_{y z} \\
\gamma_{z x} \\
\gamma_{x y}
\end{array}\right]
$$

onde $\overline{\mathbf{C}}$ é tensor constitutivo transformado.

Para o caso de EPT (KAW, 2005)

$$
\left[\begin{array}{c}
\sigma_{x} \\
\sigma_{y} \\
\tau_{x y}
\end{array}\right]=\mathbf{T}_{E P T}^{-1} \mathbf{Q} \mathbf{R}_{E P T} \mathbf{T}_{E P T} \mathbf{R}_{E P T}^{-1}\left[\begin{array}{c}
\varepsilon_{x} \\
\varepsilon_{y} \\
\gamma_{x y}
\end{array}\right]=\overline{\mathbf{Q}}\left[\begin{array}{c}
\varepsilon_{x} \\
\varepsilon_{y} \\
\gamma_{x y}
\end{array}\right]
$$

onde $\overline{\mathbf{Q}}$ é o tensor constitutivo transformado para o EPT. As matrizes de transformação $\mathbf{T}_{E P T}$ e de Reuter $\mathbf{R}_{E P T}$ são redefinidas para esta hipótese (ver Eqs. (2.28) e (2.29))(KAW, 2005)

$$
\begin{gathered}
\mathbf{T}_{E P T}=\left[\begin{array}{ccc}
c^{2} & s^{2} & 2 s c \\
s^{2} & c^{2} & -2 s c \\
-s c & s c & c^{2}-s^{2}
\end{array}\right] \\
\mathbf{R}_{E P T}=\left[\begin{array}{lll}
1 & 0 & 0 \\
0 & 1 & 0 \\
0 & 0 & 2
\end{array}\right]
\end{gathered}
$$

\subsection{Método dos Elementos Finitos}

O Método dos Elementos Finitos (MEF) é um método numérico utilizado para solucionar equações diferenciais parciais determinando uma solução $u_{h} \in U_{h}$ que aproxima-se da solução real $u \in U$, em que $U_{h}$ é o espaço das soluções admissíveis na formulação de MEF, sendo que $U_{h} \subset U$. A solução exata $u$ está geralmente fora do espaço $U_{h}$, existe portanto uma distância $d$ entre a solução aproximada $u_{h}$ e a solução exata $u$. Quando a malha do MEF é refinada, o espaço $U_{h}$ aumenta e consequentemente a distância $d$ diminui. Isso pode ser ilustrado supondo uma malha 1 , que representa um espaço $U_{h}^{1}$ que é refinada 
para obter-se as malhas $2, \ldots, i, \ldots n$, de forma que (BUCALEM; BATHE, 2011)

$$
U_{h}^{1} \subset U_{h}^{2} \subset \ldots \subset U_{h}^{i} \ldots \subset U_{h}^{n}
$$

Como pode ser observado na Fig. 2.3 , o refinamento da malha do MEF torna a solução $u_{h}$ mais próxima à solução exata $u$.

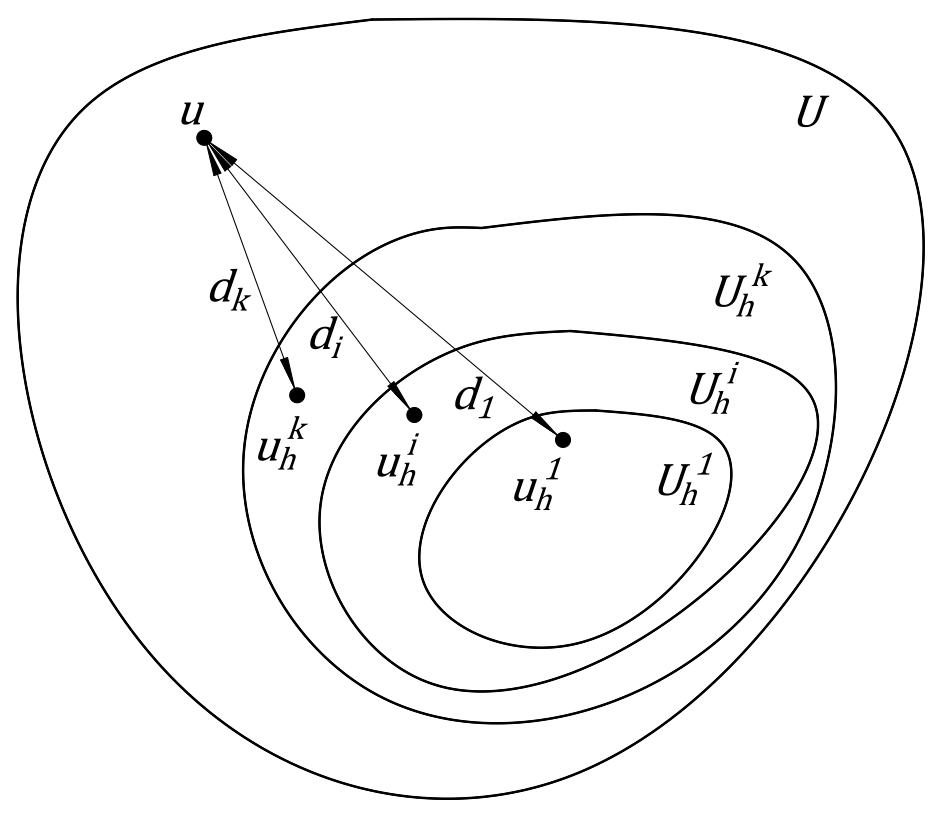

Figura 2.3 - Convergência em função do refinamento de malha.

A formulação do MEF para problemas estruturais pode ser determinada a partir do equilíbrio estático de um sólido genérico como o demonstrado na Fig. 2.4 (BATHE, 2006). Os eixos $[x, y, z]^{T}=\mathbf{x}$ representam um sistema de coordenadas fixo de onde as posições das partículas do sólido podem ser determinadas. Os deslocamentos para cada direção são definidos pelo vetor $\mathbf{u}=[u, v, w]^{T}$. Na Fig. 2.4, os deslocamentos no nó a estão representados em coordenadas locais enquanto os deslocamentos no nó $j$ estão representados em coordenadas globais. O sólido está fixado em uma área $S_{u}$ onde há um deslocamento prescrito de $\mathbf{u}^{S_{u}}$ e está sujeito a forças de tração (ou forças de superfície), forças de volume e cargas concentradas, representadas por $\mathbf{f}^{S_{f}}, \mathbf{f}^{B}$ e $\mathbf{r}_{C}^{i}$ respectivamente. $O$ índice $i$ presente em $\mathbf{r}_{C}^{i}$ representa o ponto de aplicação da carga.

Considerando a hipótese de pequenos deslocamentos, é possível definir as deformações representadas pelo tensor $\varepsilon$ (ver Seções 2.1 e 2.3) correspondentes ao vetor de deslocamentos 


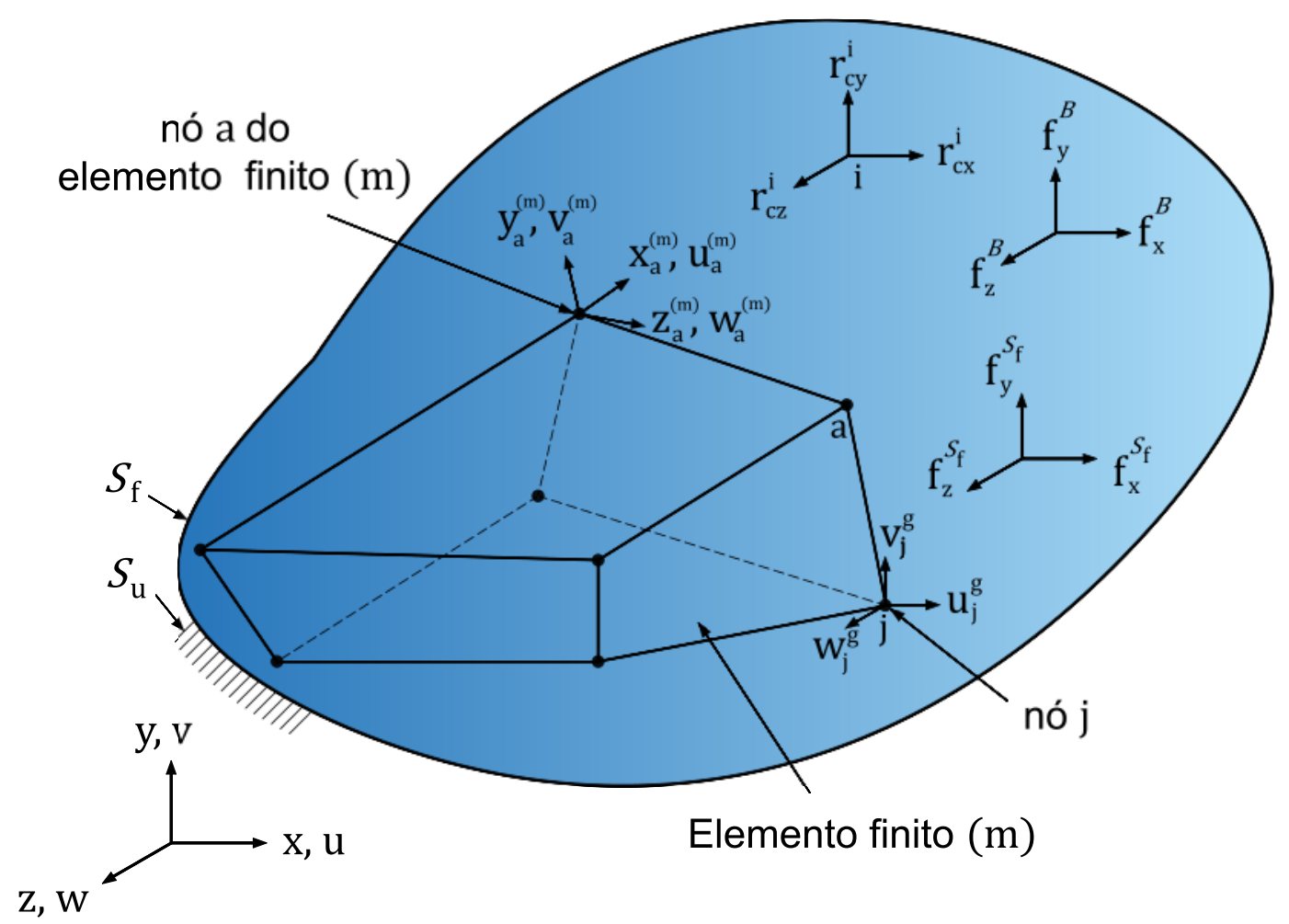

Figura 2.4 - Sólido genérico com um elemento tri-dimensional de 8 nós

u (ver Eq. (2.31)) (BATHE, 2006):

$$
\varepsilon=\left[\begin{array}{c}
\varepsilon_{x} \\
\varepsilon_{y} \\
\varepsilon_{z} \\
\gamma_{y z} \\
\gamma_{z x} \\
\gamma_{x y}
\end{array}\right]=\left[\begin{array}{c}
\frac{\partial u}{\partial x} \\
\frac{\partial v}{\partial y} \\
\frac{\partial w}{\partial z} \\
\frac{\partial v}{\partial z}+\frac{\partial w}{\partial y} \\
\frac{\partial w}{\partial x}+\frac{\partial u}{\partial z} \\
\frac{\partial u}{\partial y}+\frac{\partial v}{\partial x}
\end{array}\right]
$$

A lei material de tensão-deformação é dada pela Eq. (2.26) acrescida de uma tensão inicial $\boldsymbol{\sigma}^{I}$ no sólido (ver Eq. (2.32) ) (BATHE, 2006)

$$
\sigma=\overline{\mathbf{C}} \varepsilon+\sigma^{I}
$$

O Princípio dos Trabalhos Virtuais (PTV) estabelece que para um sólido em equilíbrio, para quaisquer pequenos deslocamentos virtuais compatíveis ( $\delta \mathbf{u}=0 \mathrm{em} S_{u}$ ), o trabalho virtual dos esforços internos $\delta W_{\text {int }}$ é igual ao trabalho virtual dos esforços externos $\delta W_{\text {ext }}$ 
(ver Eq. (2.33)) (BATHE, 2006; PIMENTA, 2008)

$$
\int_{V} \delta \boldsymbol{\varepsilon}^{T} \boldsymbol{\sigma} d V=\int_{V} \delta \mathbf{u}^{T} \mathbf{f}^{B} d V+\int_{S_{f}} \delta \mathbf{u}^{S_{f}{ }^{T}} \mathbf{f}^{S_{f}} d S+\sum_{i} \delta \mathbf{u}^{i}{ }^{T} \mathbf{r}_{C}^{i}
$$

onde $\delta \boldsymbol{\varepsilon}=\frac{\partial}{\partial \mathbf{x}}(\delta \mathbf{u})$ são deformações virtuais, $\delta \mathbf{u}^{S_{f}}$ são deslocamentos virtuais avaliados em $S_{f}$ e $\delta \mathbf{u}^{i}$ são deslocamentos virtuais equivalentes a carga concentrada $\mathbf{r}_{C}^{i}$. Neste trabalho, a solução da Eq. (2.33) é determinada utilizando o MEF por meio do programa FEniCS, onde a implementação é realizada utilizando a linguagem "Unified Form Language" (UFL), a qual é muito próxima a linguagem matemática (para maiores detalhes sobre a linguagem UFL $\operatorname{ver}($ ALNÆES et al., 2014)). No Apêndice A é demonstrado como a implementação pode ser feita em outras linguagens de programação.

\subsection{Elemento triangular e elemento tetraédrico}

No MEF, funções interpoladoras são utilizadas para determinar os valores aproximados dentro de cada elemento, usando os valores nodais. Neste trabalho utilizam-se elementos triangulares para domínios planos e elementos tetraédricos para domínios tridimensionais, os quais podem ter funções interpoladoras definidas como função da área do elemento.

Para esse tipo de elemento, define-se a aproximação das variáveis de estado $v_{e s t} \mathrm{e}$ coordenadas $(x, y)$ por meio de um mapeamento que relaciona cada ponto do elemento de um espaço paramétrico a um ponto em um espaço Euclidiano (ver Fig. 2.5) (BATHE, 2006; BUCALEM; BATHE, 2011)

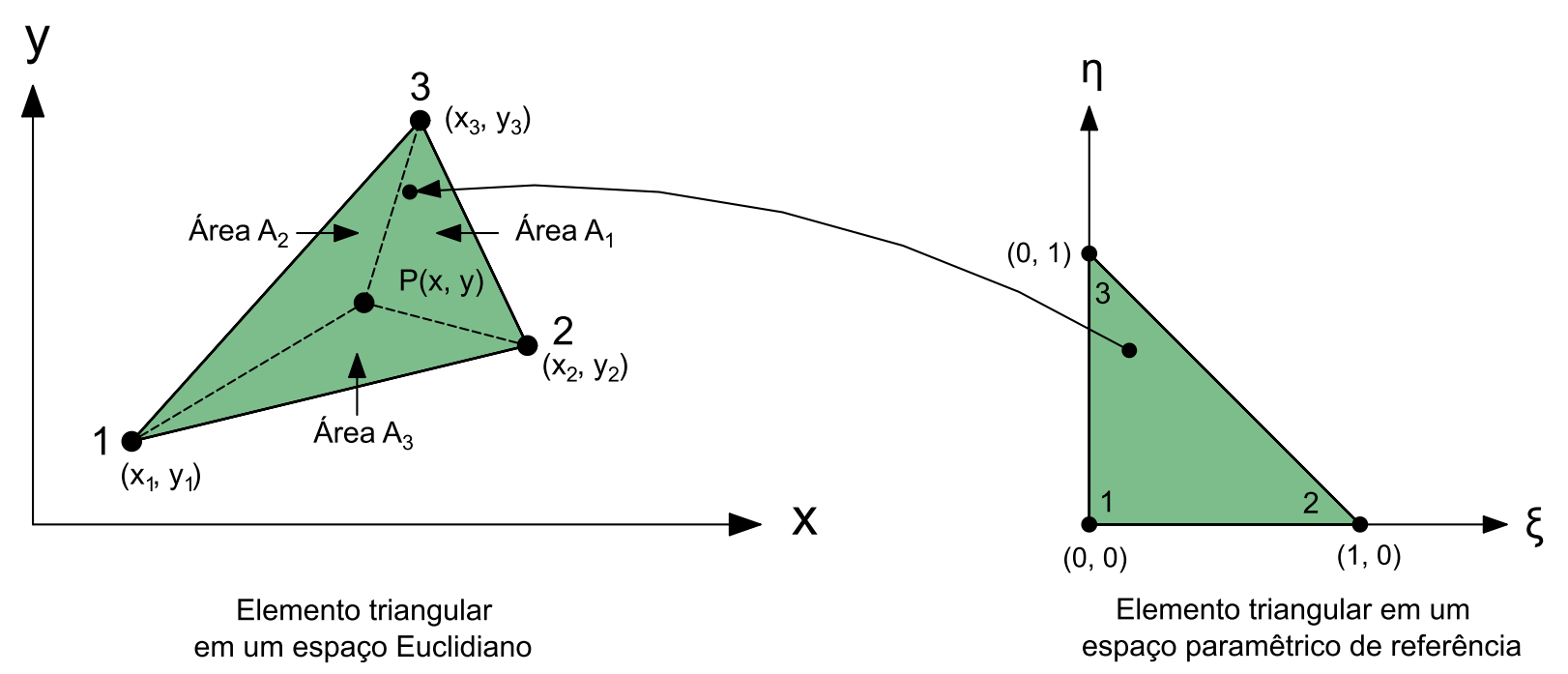

Figura 2.5 - Mapeamento entre o espaço de referência e o espaço Euclidiano.

O mapeamento entre os espaços é realizado por meio das Eqs. (2.34) a (2.36) (BATHE, 
2006; BUCALEM; BATHE, 2011)

$$
\begin{gathered}
v_{e s t}=\sum_{i=1}^{q} h_{i} \hat{v}_{i}^{e s t} \\
x \equiv \sum_{i=1}^{q} h_{i} \hat{x}_{i} \\
y \equiv \sum_{i=1}^{q} h_{i} \hat{y}_{i}
\end{gathered}
$$

onde $q$ é o número de nós do elemento triangular, $\hat{x}_{i}$ e $\hat{y}_{i}$ são as coordenadas nodais, $\hat{v}_{i}^{e s t}$ é o vetor de valores nodais das variáveis de estado e $h_{i}$ são funções de interpolação, que para o caso de interpolação linear, como a representada na Fig. 2.5, são definidas por meio da Eq. (2.37) (BATHE, 2006)

$$
\begin{gathered}
h_{1}(\xi, \eta)=\frac{A_{1}}{A}=1-\xi-\eta \\
h_{2}(\xi, \eta)=\frac{A_{2}}{A}=\xi \\
h_{3}(\xi, \eta)=\frac{A_{3}}{A}=\eta
\end{gathered}
$$

onde $\xi$ e $\eta$ são denominadas coordenadas naturais.

Para o caso de interpolação quadrática, como a representada na Fig. 2.6, as funções interpoladoras são definidas por meio da Eq. (2.38) (BATHE, 2006)

$$
\begin{gathered}
h_{1}(\xi, \eta)=(1-\xi-\eta)(1-2 \xi-2 \eta) \\
h_{2}(\xi, \eta)=\xi(2 \xi-1) \\
h_{3}(\xi, \eta)=\eta(2 \eta-1) \\
h_{4}(\xi, \eta)=4 \xi(1-\xi-\eta) \\
h_{5}(\xi, \eta)=4 \xi \eta
\end{gathered}
$$




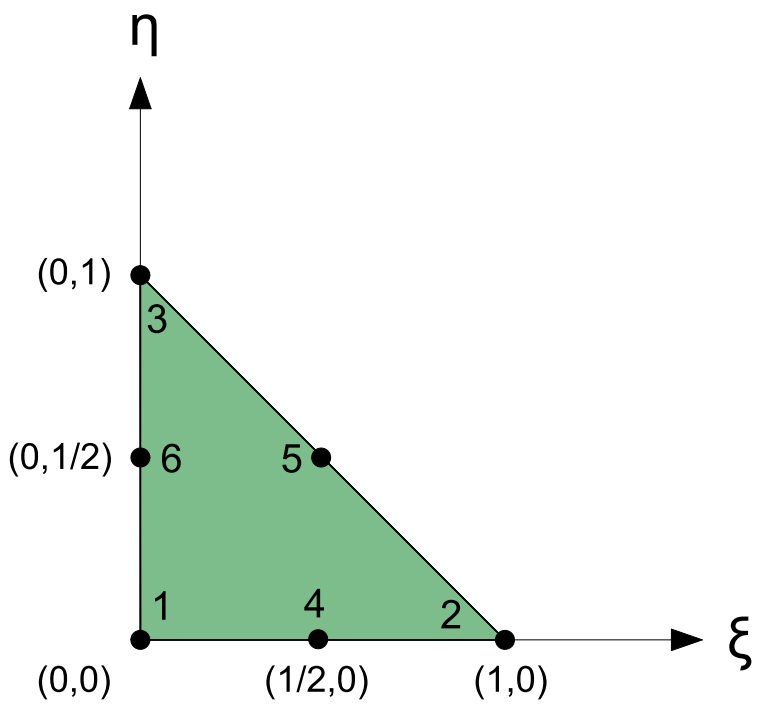

Figura 2.6 - Elemento triangular com interpolação quadrática.

$$
h_{6}(\xi, \eta)=4 \eta(1-\xi-\eta)
$$

Para o elemento tetraédrico, adotando uma abordagem semelhante para coordenadas volumétricas (ver Fig. 2.7), pode-se definir as funções de interpolação, para o caso linear, por meio da Eq. (2.39) (BATHE, 2006)

$$
\begin{gathered}
h_{1}=1-\xi-\eta-\zeta \\
h_{2}=\xi \\
h_{3}=\eta \\
h_{4}=\zeta
\end{gathered}
$$

onde $\zeta$ é a terceira coordenada natural.

A relação entre as derivadas parciais das coordenadas cartesianas e as derivadas parciais das coordenadas naturais é dada por meio do operador Jacobiano J (ver Eqs. (2.40) e (2.41)) (BATHE, 2006)

$$
\frac{\partial}{\partial \mathbf{x}}=\mathbf{J}^{-1} \frac{\partial}{\partial \boldsymbol{\xi}}
$$




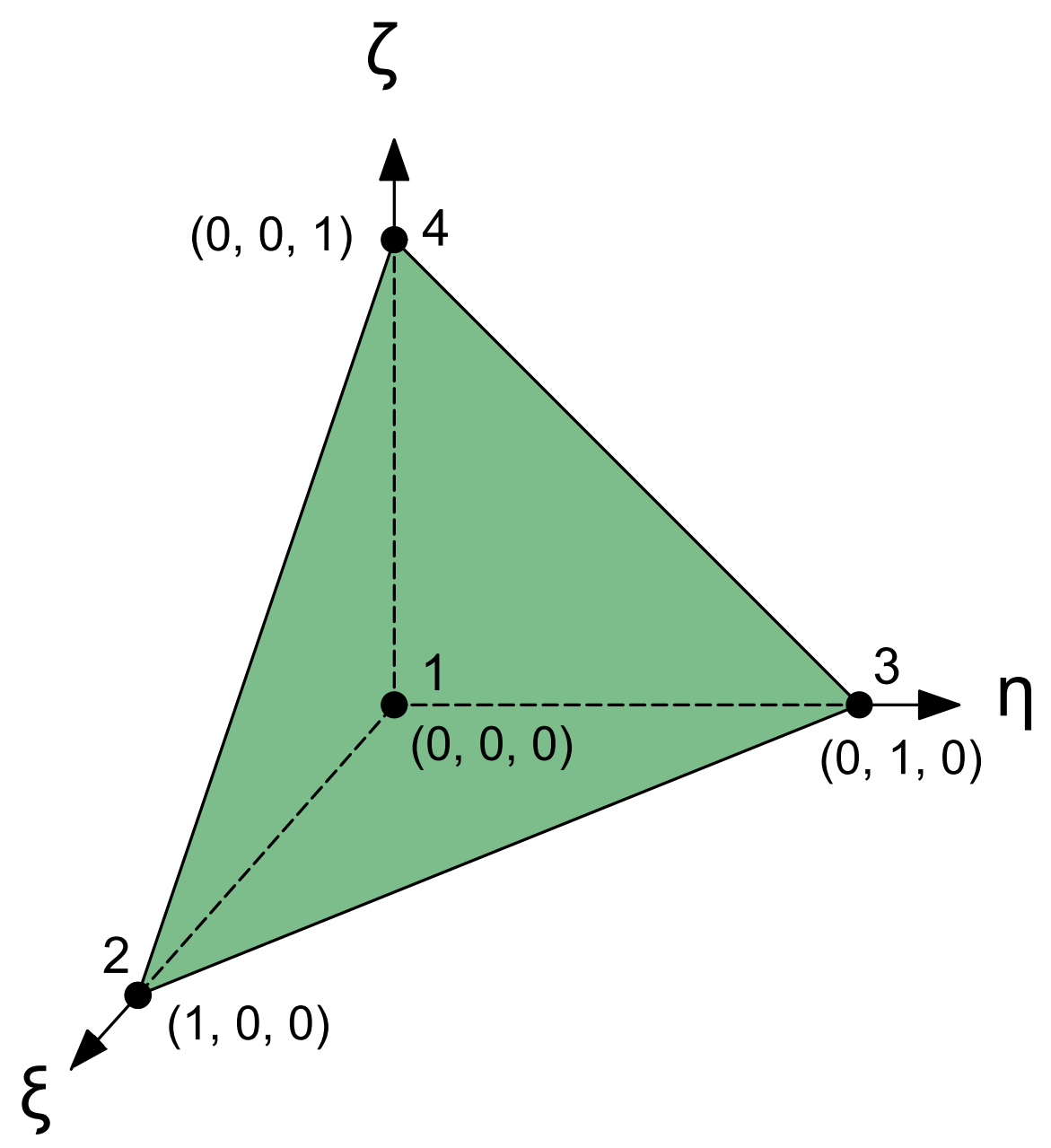

Figura 2.7 - Coordenadas naturais de um elemento tetraédrico de 4 nós.

$$
\mathbf{J}=\left[\begin{array}{lll}
\frac{\partial x}{\partial \xi} & \frac{\partial y}{\partial \xi} & \frac{\partial z}{\partial \xi} \\
\frac{\partial x}{\partial \eta} & \frac{\partial y}{\partial \eta} & \frac{\partial z}{\partial \eta} \\
\frac{\partial x}{\partial \zeta} & \frac{\partial y}{\partial \zeta} & \frac{\partial z}{\partial \zeta}
\end{array}\right]
$$

O operador Jacobiano $\mathbf{J}$ também é utilizado para definir o volume diferencial $d V$ em termos de coordenadas naturais (ver Eq. (2.42))

$$
d V=\operatorname{det} \mathbf{J} d \xi d \eta d \zeta
$$




\section{OTIMIZAÇÃO TOPOLÓGICA}

A OT é uma técnica que combina métodos numéricos, geralmente o MEF, com algoritmos de otimização para determinar uma distribuição de material no interior de um domínio fixo de projeto, com o objetivo de otimizar o desempenho da estrutura respeitando determinadas restrições impostas. Embora a OT seja atualmente aplicada em diversos tipos de problemas, sua primeira implementação foi utilizada para se determinar a máxima rigidez em estruturas considerando restrição de volume (BENDSøE; KIKUCHI, 1988). A abordagem proposta por Bendsøe e Kikuchi (1988) utiliza o método da homogeneização, o que consequentemente, apresenta estruturas porosas como resultado da otimização. Um ano depois, Bendsøe (1989) propõe o método das densidades, que consiste em determinar a distribuição de pseudo-densidades $\bar{\rho}$, que podem variar entre 0 (vazio) e 1 (sólido), em um domínio fixo (ver Fig. 3.1). O método das densidades demanda a utilização de um modelo de material, entre os quais o mais conhecido é denominado "'Solid Isotropic Material with Penalization" (SIMP)", que apesar de ter sido introduzido por Bendsøe (1989), recebeu essa nomenclatura em (ROZVANY; ZHOU; BIRKER, 1992).

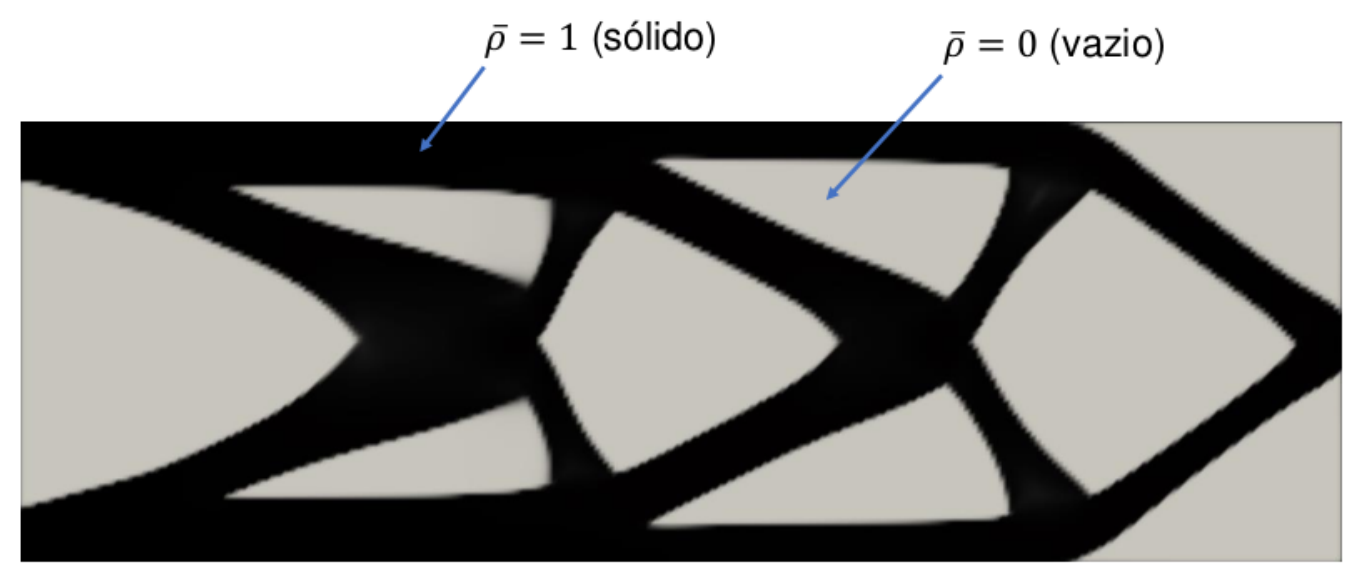

Figura 3.1 - Resultado típico de OT utilizando o método das densidades.

\subsection{Modelo de Material SIMP}

O modelo SIMP é utilizado para a solução de diferentes problemas na literatura como: projeto de mecanismos flexíveis (SIGMUND, 1997), projeto de estruturas com não linearidade geométrica (BUHL; PEDERSEN; SIGMUND, 2000), atuadores multi-físicos (SIGMUND, 2001), estruturas de cristais fotônicos (JENSEN; SIGMUND, 2004), problemas 
baseados em confiabilidade (KHARMANDA et al., 2004), estruturas sujeitas a vibrações por radiação sonora (OLHOFF; DU, 2006), estruturas sujeitas a cargas seguidoras (CHEN; KIKUCHI, 2001), estruturas de cascas (MAUTE; RAMM, 1997) e estruturas considerando elasto-plasticidade (MAUTE; SCHWARZ; RAMM, 1998). Maiores detalhes sobre trabalhos que utilizam o modelo SIMP podem ser encontrados no trabalho de (ROZVANY, 2009).

Utilizando o modelo SIMP, pode-se determinar a partir de um domínio fixo $V$, um subdomínio $V^{\text {mat }}$ que representa a distribuição otimizada de material (ver Fig. 3.2), em que o tensor constitutivo é determinado por meio da Eq. (3.1) (BENDSØE; SIGMUND, 2003)

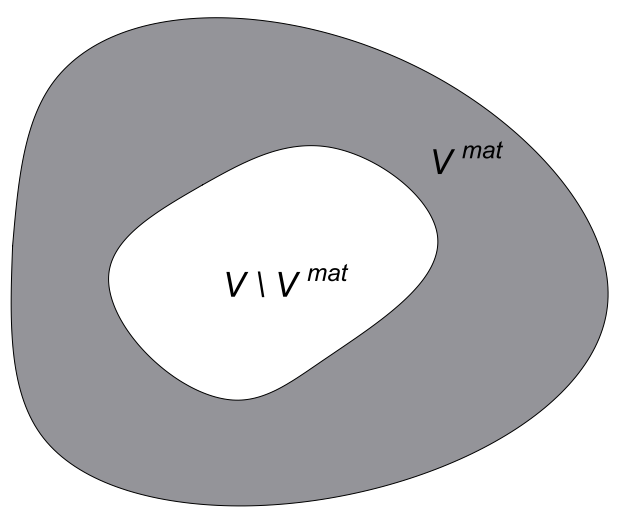

Figura 3.2 - Definição do domínio de projeto

$$
\begin{aligned}
\overline{\mathbf{C}}(\mathbf{x})=1 V^{\text {mat }} \overline{\mathbf{C}}^{0}(\mathbf{x}), \quad \mathbf{1} V^{\text {mat }}= \begin{cases}1 & \text { se } \mathbf{x} \in V^{\text {mat }} \\
0 & \text { se } \mathbf{x} \in V \backslash V^{\text {mat }}\end{cases} \\
\int_{V} \mathbf{1} V^{\text {mat }} d V \leq V
\end{aligned}
$$

onde a inequação expressa na Eq. (3.1b), representa o limite de material disponível para a otimização e o tensor $\overline{\mathbf{C}}^{0}(\mathbf{x})$ é o tensor constitutivo de um determinado material.

A definição expressa em Eq. (3.1) representa um problema de otimização de variáveis discretas, que quando definido no espaço contínuo, pode apresentar múltiplas soluções ou não apresentar solução alguma. Este problema pode ser contornado substituindo as variáveis discretas por variáveis contínuas e utilizando uma penalização para que ao fim da otimização, seus valores se aproximem de 0 e 1. A determinação das variáveis contínuas é realizada por meio de uma função que pode ser interpretada como uma pseudo-densidade $\bar{\rho}$ do material(ver Eq. (3.2)) (BENDSøE; SIGMUND, 2003).

$$
\overline{\mathbf{C}}(\mathbf{x})=\bar{\rho}(\mathbf{x})^{p} \overline{\mathbf{C}}^{0}(\mathbf{x}), \quad p>1
$$




$$
\int_{V} \bar{\rho} d V \leq V ; \quad 0<\bar{\rho}_{\min } \leq 0 \leq \bar{\rho} \leq 1, \quad \mathbf{x} \in V
$$

Nesta formulação, a pseudo-densidade $\bar{\rho}$ é a variável de projeto, a qual interpola os valores do tensor $\overline{\mathbf{C}}$ entre 0 e $\overline{\mathbf{C}}^{0}$ e $p$ é o penalizador, que tem como função aproximar os valores de $\bar{\rho}$ aos valores discretos 0 e 1 (ver Fig. 3.3) (BENDS $\varnothing E ;$ SIGMUND, 2003).

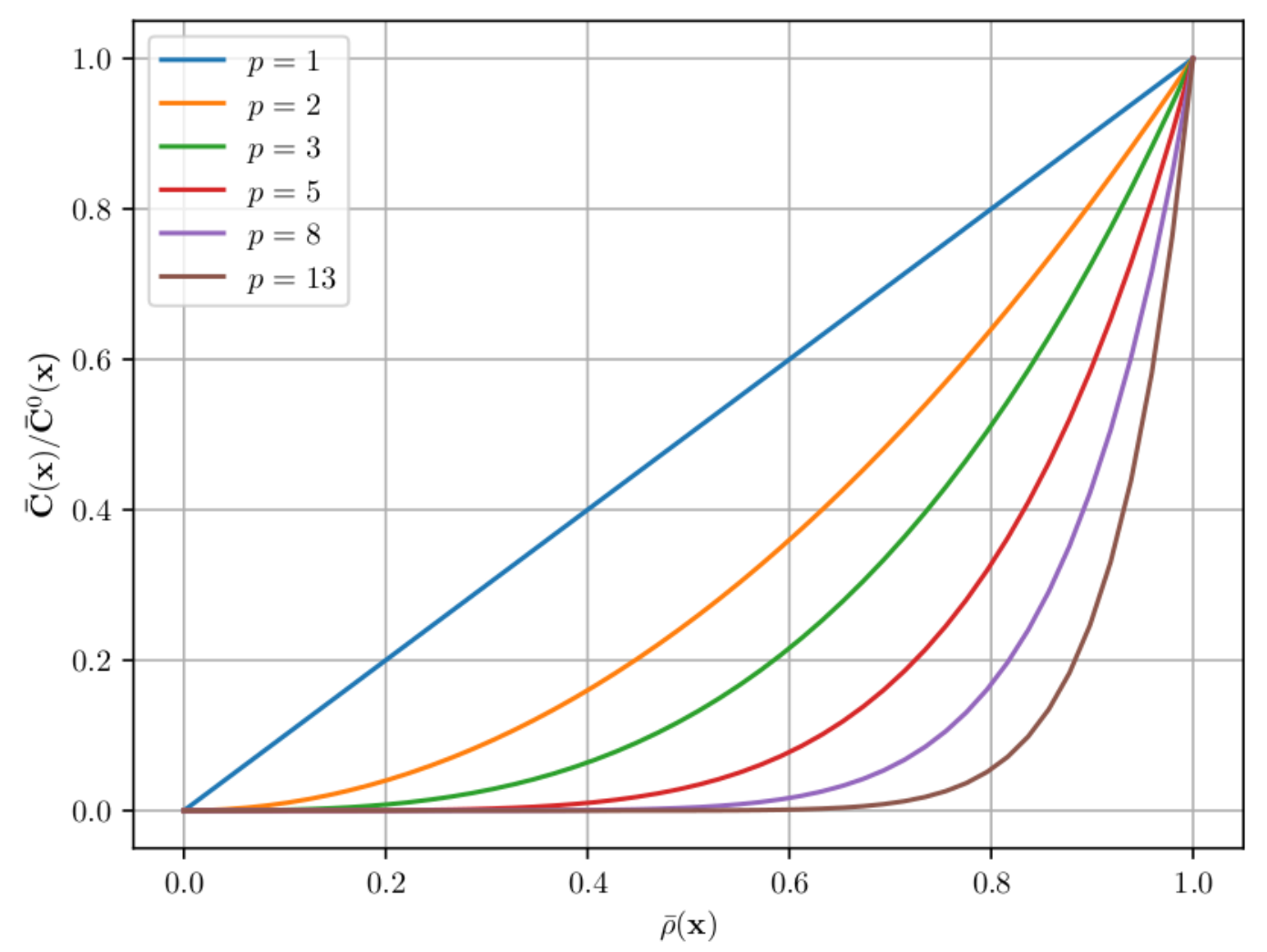

Figura 3.3 - Influência do penalizador $p$

Como pode ser observado na Fig. 3.3, a relaxação do problema de otimização permite que a pseudo-densidade $\bar{\rho}$ assuma valores entre 0 e 1 . Estes valores de pseudo-densidades intermediárias representam indesejáveis escalas de cinza no resultado do problema de otimização (ver Fig. 3.4). Diversos esquemas de penalização são utilizados na literatura para tratar o problema de escalas de cinza, assumindo-se que a topologia otimizada obtida como resultado do problema relaxado é semelhante a do problema discreto 0-1 (LAU; DU; LIM, 2001). Um tipo de penalização consiste em aumentar (ou diminuir dependendo do modelo adotado) gradativamente os valores de $p$ durante o processo de otimização, o que é conhecido como Método de Continuação (BENDSøE; SIGMUND, 2003). Esta alteração no valor do penalizador $p$ evita que a solução da OT estacione em um mínimo local, o que pode ocorrer, no caso do modelo SIMP, para funções onde $p>1$, pois de forma geral, são 
casos não convexos (ver Fig. 3.5).

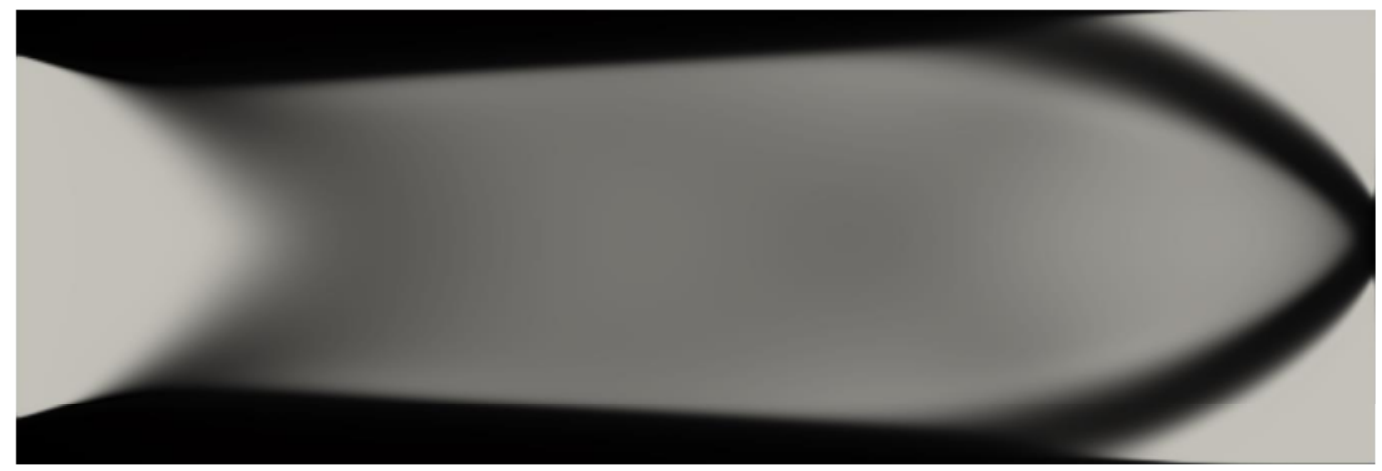

Figura 3.4 - Representação de uma viga engastada otimizada contendo escala de cinza.

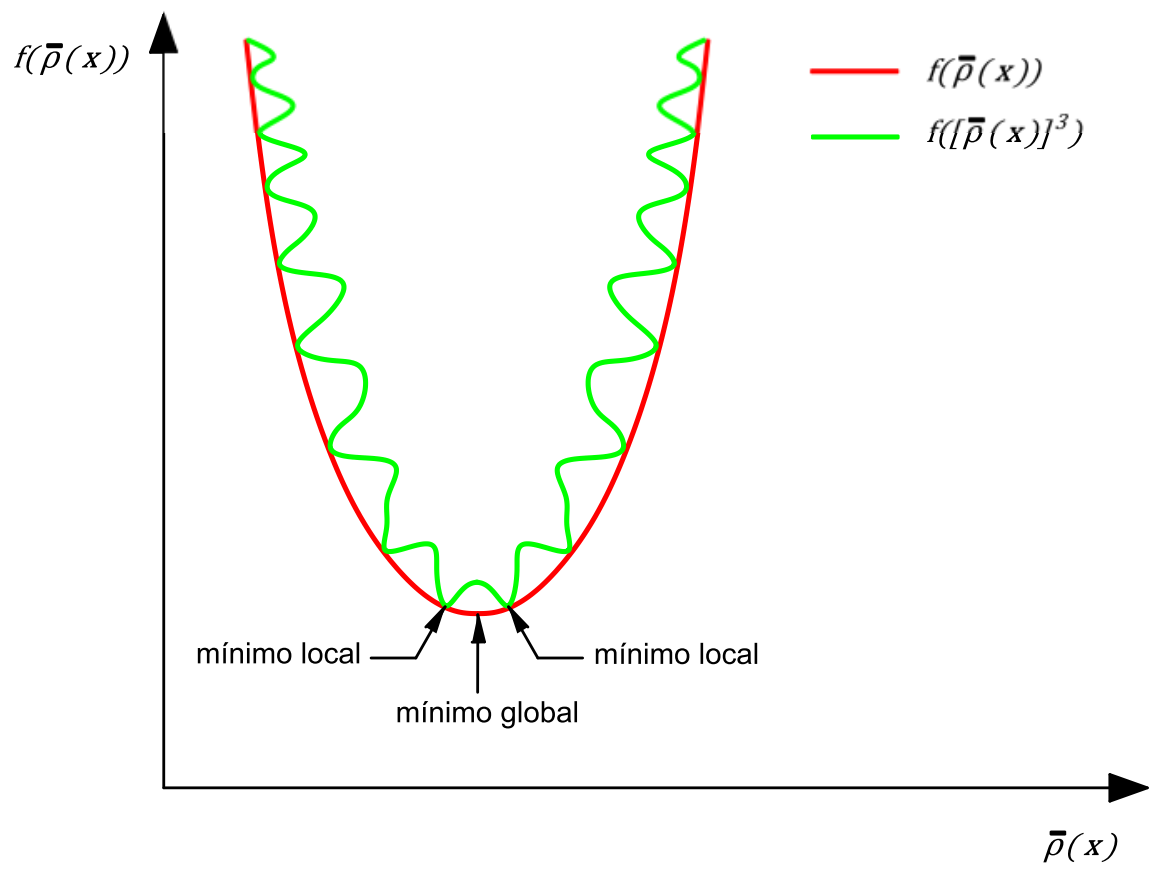

Figura 3.5 - Representação esquemática da influência da penalização na solução do problema de otimização topológica.

Para o exemplo representado pela Fig. 3.5, tem-se uma função $f$ que é convexa quando $f=f(\bar{\rho}(\mathbf{x}))$ e mal comportada no caso em que $f=f\left([\rho(\overline{\mathbf{x}})]^{3}\right)$. Para este caso específico, recomenda-se iniciar a otimização com $p=1$ até que a convergência seja atingida e após isto, aumentar gradualmente o valor de $p$ até que um menor mínimo local seja atingido. 


\subsection{Instabilidade de tabuleiro}

Um problema recorrente na utilização da OT é a Instabilidade de tabuleiro que ocorre principalmente ao se utilizar MEF baseado em deslocamentos, especialmente com elementos de primeira ordem (ZHOU; SHYY; THOMAS, 2001; BENDSøE; SIGMUND, 2003). Este tipo de instabilidade se caracteriza pela surgimento de um padrão similar a um tabuleiro de xadrez em regiões onde se alternam a presença e ausência de material (ver Fig. 3.6). Este padrão de distribuição de material não representa uma estrutura otimizada e surge devido a ruídos numéricos causados por erros na formulação de MEF (ZHOU; SHYY; THOMAS, 2001). O surgimento destes padrões ocorre pelo fato de que regiões que apresentam esta configuração possuem rigidez mais elevada que regiões homogêneas com o mesmo volume de material, principalmente onde há cisalhamento (BENDSøE; SIGMUND, 2003).

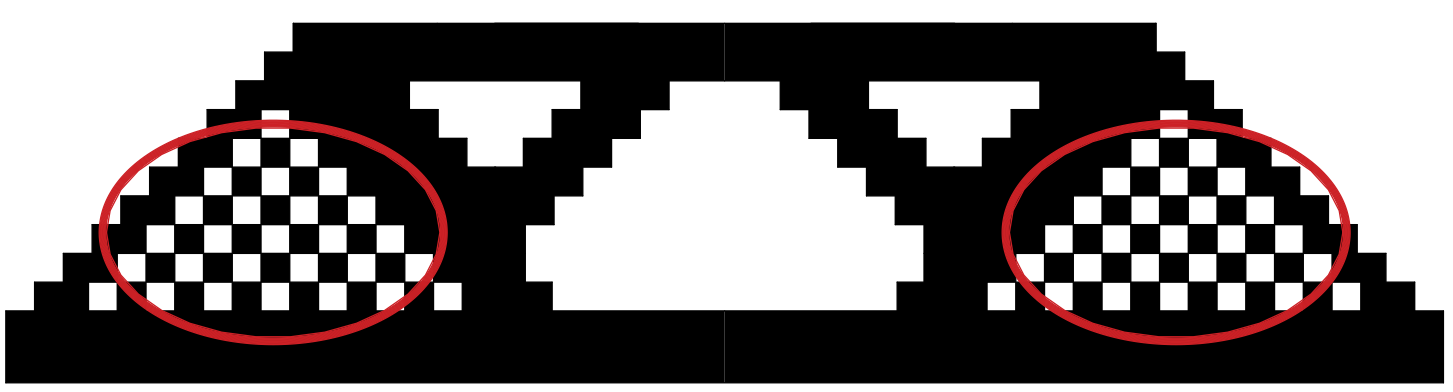

Figura 3.6 - Padrão de instabilidade de tabuleiro em uma viga simplesmente apoiada.

A instabilidade de tabuleiro também pode ser interpretada por outra perspectiva. 0 problema de OT envolve dois campos distintos, o de pseudo-densidade e o de deslocamentos, o que implica em um problema de variacional misto e em função disto, surgem os padrões de tabuleiro (JOG; HABER; BENDSØE, 1994; DIAZ; SIGMUND, 1995; JOG; HABER, 1996). É possível mitigar este tipo de instabilidade aumentado a ordem de interpolação do elemento utilizado na malha de MEF, o que implica em uma solução de compromisso, pois o uso de elementos de ordens superiores aumenta consideravelmente o custo computacional. Para o caso do SIMP, o problema de instabilidade tabuleiro é evitado utilizando-se elementos de 8 ou 9 nós, mas somente se o penalizador for pequeno o suficiente (para mais detalhes consultar (DIAZ; SIGMUND, 1995)). Uma alternativa viável do ponto de vista computacional é a utilização de um método de filtragem ou controle de gradiente da pseudo-densidade, que além de tratar o problema de instabilidade de tabuleiro, também é eficiente para o problema de dependência de malha.

\subsection{Dependência de malha}

Como citado na Seção 2.4, quanto mais refinada a malha do MEF, mais próxima a solução aproximada $u_{h}$ obtida estará da solução real $u$. Por este motivo, faz sentido supor que o resultado de OT para uma malha mais refinada, resulta em uma mesma 
topologia com fronteiras mais definidas, porém, a consequência de uma maior discretização, considerando as mesmas condições de contorno no mesmo domínio fixo, é o aparecimento de mais membros na estrutura (ver Fig. 3.7). Este efeito, caracterizado pela obtenção de diferentes topologias em função da discretização, recebe o nome de Dependência de malha (SIGMUND; PETERSSON, 1998).

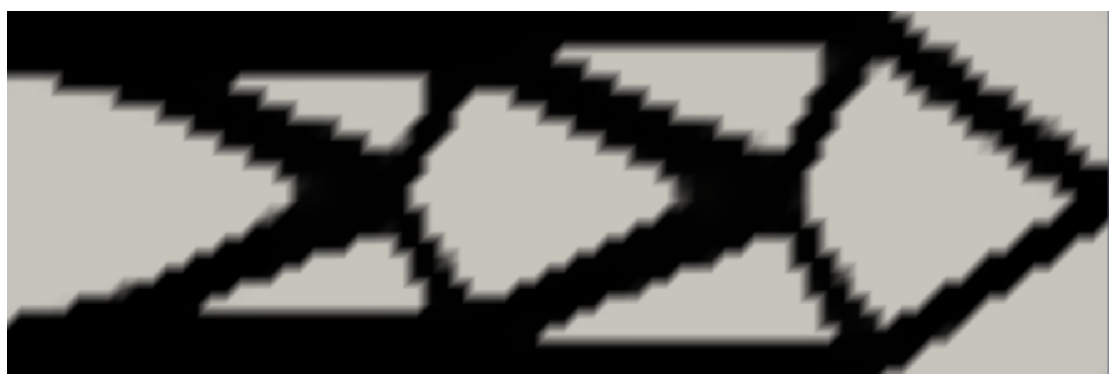

(a) Malha $75 \times 25$

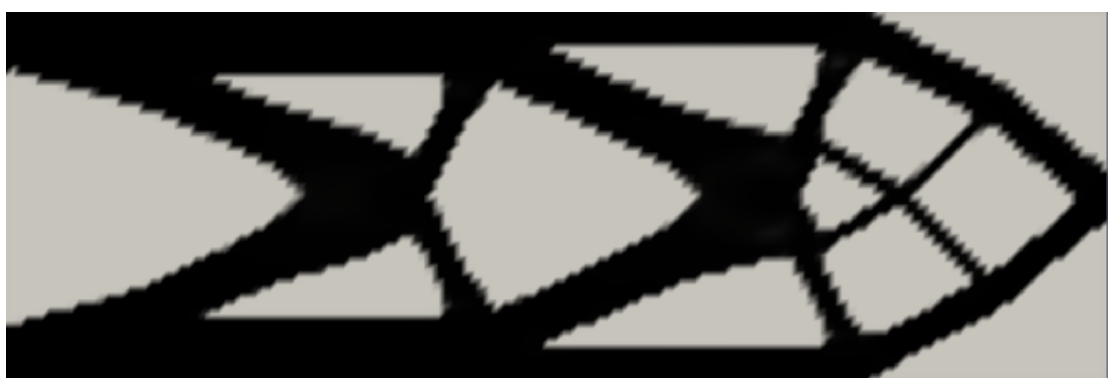

(b) Malha $150 \times 50$

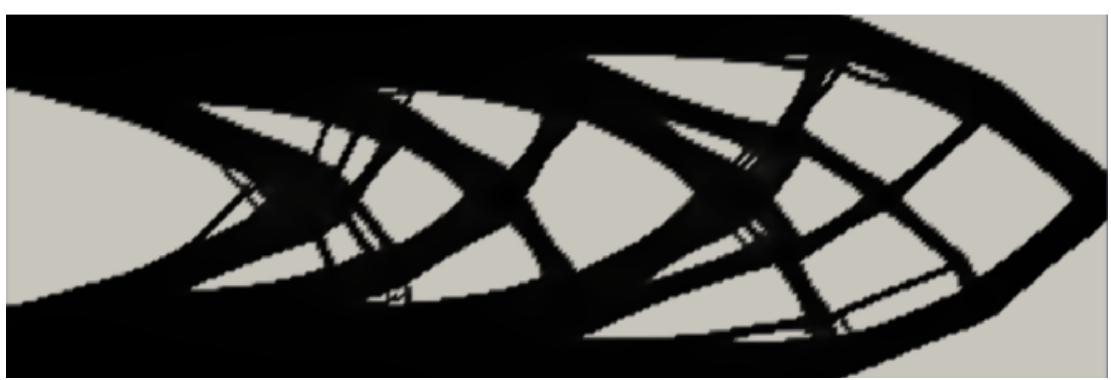

(c) Malha $300 \times 100$

Figura 3.7 - Efeito da dependência de malha

De acordo com Sigmund e Petersson (1998), os problemas de dependência de malha podem ser divididos em duas categorias, não existência da solução, caracterizada pelo fato de sempre se obter resultados diferentes de acordo com o refinamento da malha e não unicidade da solução, que ocorre quando o problema possui múltiplos mínimos, como em um caso de tração uniaxial onde uma só barra é um resultado tão bom quanto várias barras de mesmo volume (ver Fig. 3.8 )

Em ambos os casos, pode-se dizer que o problema da dependência de malha surge como consequência de um problema mal posto. É possível solucionar este problema por meio da imposição de restrições, que podem ser globais ou locais, que controlem a variação 


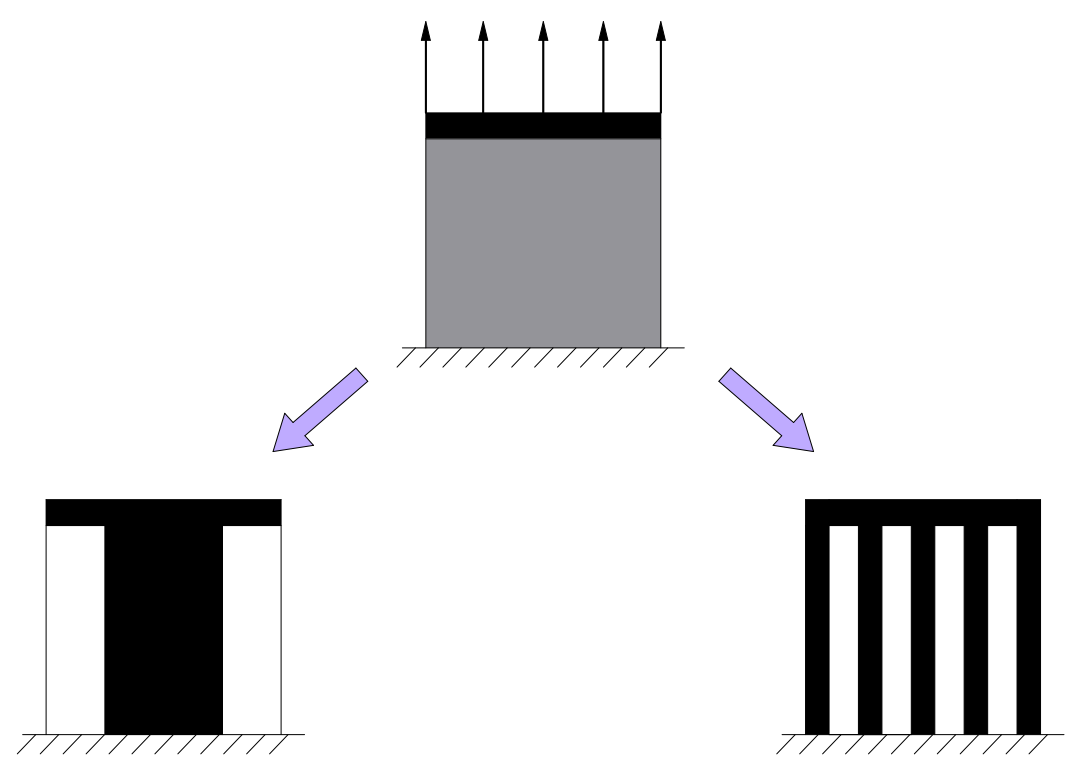

Figura 3.8 - Exemplo de não unicidade da solução.

da densidade. Segundo Sigmund e Petersson (1998), existem quatro possíveis abordagens para a aplicação destas restrições, são elas, controle de perímetro, restrições de gradientes globais ou locais, e aplicação de métodos de filtragem. O controle de perímetro consiste em restringir a soma dos perímetros das fronteiras internas e externas, limitando o número de possíveis furos na topologia final. Esta limitação do perímetro é realizada por meio da imposição de um limite superior na variação total da pseudo-densidade (SIGMUND; PETERSSON, 1998; BENDSøE; SIGMUND, 2003). De acordo com Zhou, Shyy e Thomas (2001), este método é uma maneira eficiente de se obter resultados independentes da malha, pois é necessário introduzir apenas mais uma restrição no problema de otimização.

O método do perímetro é utilizado por Haber, Jog e Bendsøe (1996), onde restrições superiores e inferiores são impostas no problema de otimização garantindo que o mesmo seja bem posto. (DUYSINX, 1997) apresenta um método de programação matemática aplicado a otimização topológica, utilizando restrições de perímetro para regularizar a distribuição de material. No entanto, a restrição do perímetro pode introduzir múltiplos mínimos locais, portanto, não garante a unicidade da solução. Além disso, a restrição utilizada neste método pode, em alguns casos, causar flutuações nos valores das variáveis de projeto.

A imposição da restrição global de gradientes é realizada por meio da norma da função $\bar{\rho}$ no espaço de Sobolev $H^{1}$ (V) (BENDS $\varnothing E$; SIGMUND, 1995; SIGMUND; PETERSSON, 1998). A existência da solução é provada em Bendsøe e Sigmund (1995).

A restrição local de gradientes é caracterizada pela imposição de um limite na variação local nas derivadas da função $\bar{\rho}$ (SIGMUND; PETERSSON, 1998; BENDSØE; SIGMUND, 
2003). A prova da existência de solução e a implementação numérica deste tipo de restrição são apresentadas em (PETERSSON; SIGMUND, 1998), no entanto, apesar desta técnica ser capaz de mitigar ou mesmo eliminar problemas numéricos como a dependência de malha ou a instabilidade de tabuleiro, a implementação deste esquema demanda a inserção de um grande número de restrições, o que torna o custo computacional elevado para problemas práticos (SIGMUND; PETERSSON, 1998).

\subsection{Métodos de filtragem}

Outra eficiente abordagem para tratar o problema de dependência de malha são os métodos de filtragem que consistem em substituir a dependência pontual das propriedades do material por uma regularização do campo de densidades (ZHOU; SHYY; THOMAS, 2001). O filtro de densidades foi proposto por Bruns e Tortorelli (2001) e a existência de solução utilizando o modelo de material SIMP foi demonstrada no trabalho de Bourdin (2001). A ideia é baseada em técnicas de blurring utilizadas para o processamento de imagens e consiste em modificar a densidade de uma célula, e consequentemente a rigidez do elemento, para torná-la dependente de um campo de densidades em sua vizinhança. $A$ aplicação do filtro consiste na utilização de um produto de convolução entre uma função de filtragem e a pseudo-densidade (ver Eqs. (3.3) e (3.4) ) (LAZAROV; SIGMUND, 2011).

$$
\begin{gathered}
\tilde{\rho}(\mathbf{x})=(f * \bar{\rho})(\mathbf{x})=\int_{\mathbb{B}_{R}} f(\mathbf{x}-\mathbf{y}) \bar{\rho}(\mathbf{y}) d \mathbf{y} \\
\int_{\mathbb{B}_{R}} f(\mathbf{x}) d \mathbf{x}=1
\end{gathered}
$$

onde $\mathbb{B}_{R}$ é um círculo em um domínio plano e uma esfera em um caso de três dimensões, com raio $R$ e centro na posição $\mathbf{x}$.

Neste trabalho um filtro de densidades proposto por (LAZAROV; SIGMUND, 2011) é utilizado, em função da eficiência do método e da facilidade de implementação no software FEniCS. Neste método, ao invés de utilizar a integral de convolução de forma explicita, utiliza-se a solução de uma equação modificada de Helmholtz com condições de contorno naturais homogêneas (ver Eqs. (3.5) e (3.6))

$$
\begin{gathered}
-r^{2} \nabla^{2} \tilde{a}+\tilde{a}=a \\
\frac{\partial \tilde{a}}{\partial \mathbf{n}}=0
\end{gathered}
$$


onde a representa uma variável de projeto, ã representa a variável de projeto filtrada e $r$ é um parâmetro de comprimento com função similar ao $R$ das Eqs. (3.3) e (3.4). Avaliando a Eq. (3.5) pode-se perceber que se $r=0$, a variável filtrada ã será igual a variável $a$, porém, quanto maior o valor de $r$, maior é a influência do gradiente da variável filtrada na solução da equação. A Fig. 3.9 demonstra a representação da solução da equação modificada de Helmholtz para diferentes valores de $r$ em um domínio unidimensional.

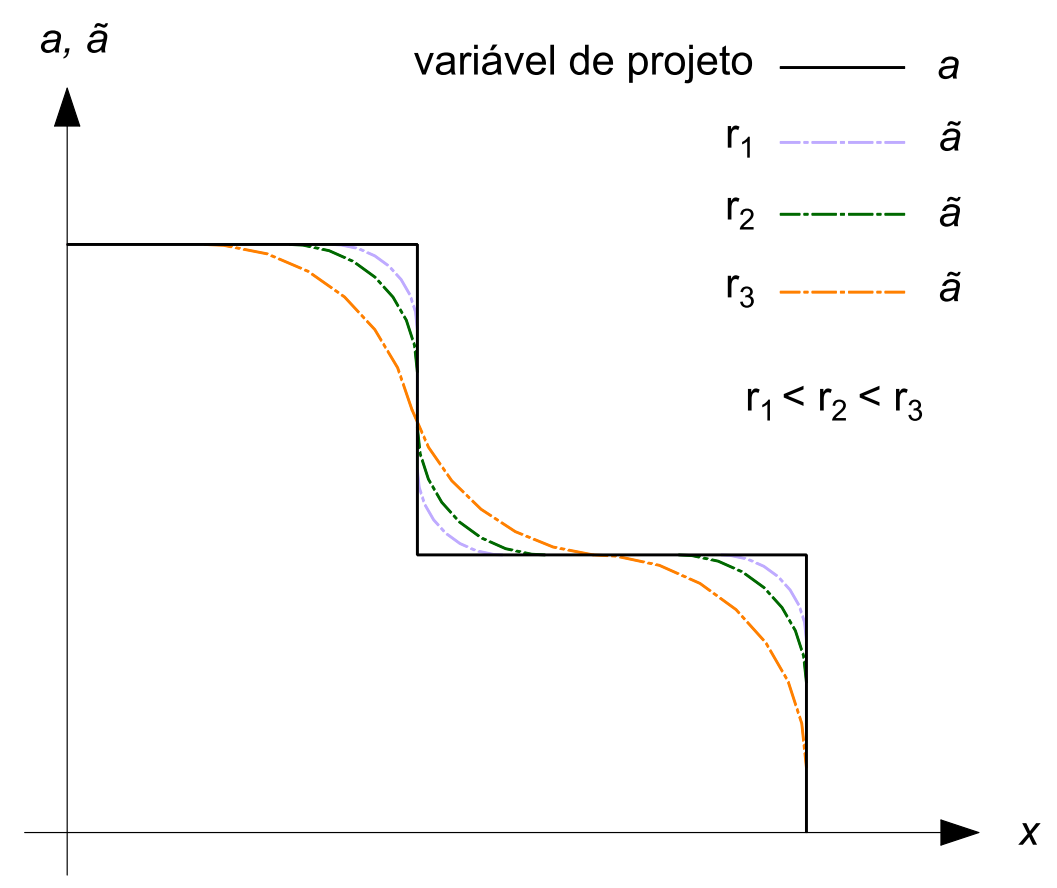

Figura 3.9 - Efeito da alteração do parâmetro $r$ na solução da equação modificada de Helmholtz.

As Eqs. (3.5) e (3.6) configuram a Forma Forte do problema. Para determinar a solução aproximada utilizando o MEF, é necessário desenvolver a Forma Fraca, multiplicando ambos os lados da Fig. 3.9 por uma função teste $v$ e integrando no domínio (ver Eq. (3.7))

$$
\int_{V}-r^{2} \nabla^{2} \tilde{a} \cdot v d V+\int_{V} \tilde{a} \cdot v d V=\int_{V} a \cdot v d V
$$

Integrando por partes e impondo a condição de contorno natural dada pela Eq. (3.6) pode-se reescrever a forma fraca Eq. (3.7) como

$$
\int_{V} r^{2} \nabla \tilde{a} \cdot \nabla v d V+\int_{V} \tilde{a} \cdot v d V=\int_{V} a \cdot v d V
$$

A solução da equação modificada de Helmholtz (ver Eq. (3.8)) é obtida utilizando o software FEniCS, com a mesma malha utilizada para se obter a aproximação do deslocamento 
u. Para a implementação em outras linguagens de programação, consultar o Apêndice B.

\subsection{Modelos para otimização de orientação de fibras}

Como explorado na Seção 1.1, os modelos OFDN e MIAFIB apresentam vantagens em relação a outros métodos. Ambos utilizam apenas uma variável de projeto por elemento (ou nó da malha de MEF, dependendo da formulação do problema) e, cada qual a sua maneira, conseguem evitar problemas de múltiplos mínimos locais. Neste capítulo são apresentadas as formulações para um modelo baseado no OFDN, denominado OFDN-m, e para o MIAFIB.

\subsubsection{Método Otimização de Fibra por Distribuição Normal modificado (OFDN-m)}

Os chamados Métodos de Ângulos Discretos, onde ângulos candidatos são escolhidos antes do processo de otimização, utilizam o mesmo princípio, que consiste em determinar um tensor constitutivo efetivo $\overline{\mathbf{C}}_{e}$ por meio da soma ponderada dos tensores constitutivos $\overline{\mathbf{C}}_{i}$ de cada ângulo candidato $i$ (ver Eq. (3.9) ) (KIYONO; SILVA; REDDY, 2017)

$$
\overline{\mathbf{C}}_{e}=\sum_{i=1}^{n_{c}} w_{i} \overline{\mathbf{C}}_{i}
$$

onde $n_{c}$ é o número de ângulos candidatos e $w_{i}$ são as funções de ponderação.

A diferença entre os métodos de ângulos discretos está na forma como são parametrizadas as funções de ponderação. Duas condições precisam ser satisfeitas para garantir que os resultados possuam propriedades físicas realísticas, as funções $w_{i}$ devem possuir valores entre 0 e 1 e a soma de todas elas deve ser igual a 1 (ver Eqs. (3.10) e (3.11)) (STEGMANN; LUND, 2005; KIYONO; SILVA; REDDY, 2017)

$$
\begin{gathered}
0 \leq w_{i} \leq 1 \\
\sum_{i=1}^{n_{c}} w_{i}=1
\end{gathered}
$$

O tensor constitutivo efetivo $\overline{\mathbf{C}}_{e}$ deve assumir o valor de um dos tensores $\overline{\mathbf{C}}_{i}$ candidatos. Para isso, apenas uma função $w_{i}$ deve assumir o valor de 1 , enquanto todas as outras devem ser iguais a 0. (STEGMANN; LUND, 2005) propõem que a função de ponderação 
seja a demonstrada na Eq. (3.12)

$$
w_{i}=\frac{\hat{w}_{i}}{\sum_{k=1}^{n_{c}} \hat{w}_{k}}
$$

onde a função de distribuição normal modificada $\hat{w}_{i}$, representada na Eq. (3.13), é utilizada

$$
\hat{w}=e^{-\frac{\left(\theta-\theta_{c}^{i}\right)^{2}}{2 p_{\theta}^{2}}}
$$

A variável $\theta$, presente na Eq. (3.13), é a variável de orientação da fibra (ver Fig. 3.10), $\theta_{c}^{i}$ é o ângulo candidato $i$ e $p_{\theta}$ é o penalizador.

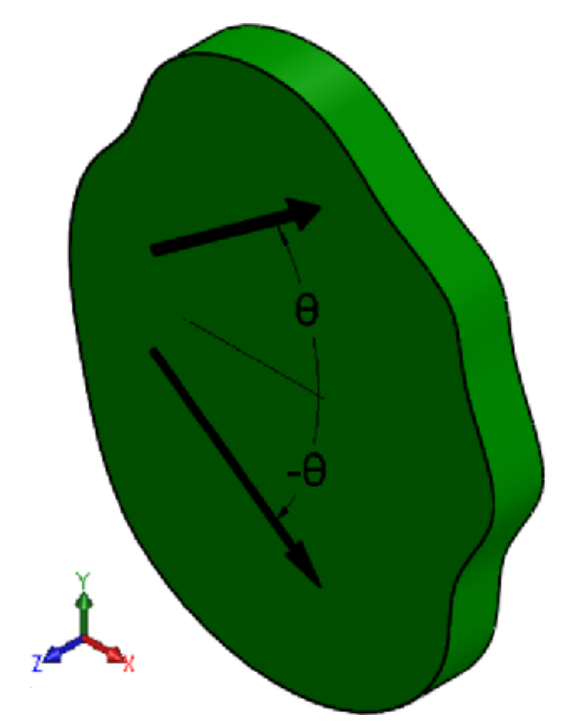

Figura 3.10 - Definição do ângulo $\theta$

O modelo OFDN-m foi proposto como consequência deste trabalho (SILVA et al., 2019) e tem como principal diferença o fato de utilizar diretamente o ângulo da fibra como variável de projeto (ver Eq. (3.13)), diferentemente do modelo OFDN (KIYONO; SILVA; REDDY, 2017), que tem como variável um número inteiro que representa a posição de cada candidato em uma lista de candidatos. Esta modificação no espaço de solução permite a determinação de diferentes sensibilidades de acordo com os intervalos entre os ângulos candidatos, tornando possível priorizar inicialmente determinados ângulos. O parâmetro $\theta_{c}^{i}$ determina a posição do pico da função $\hat{w}$, enquanto o penalizador controla quão suave é esta função ver (Fig. 3.11).

A Fig. 3.11b demonstra que valores menores de $p_{\theta}$ conduzem $\hat{w}$ para $1 \mathrm{em}$ um determinado ângulo e 0 para todos os outros. No entanto, não deve-se iniciar o processo de otimização com valores pequenos de $p_{\theta}$, pois isto pode causar instabilidades numéricas levando a um problema de múltiplos mínimos locais. Portanto, se faz necessário a utilização de um método de continuação que se inicia com um valor suficientemente grande para 


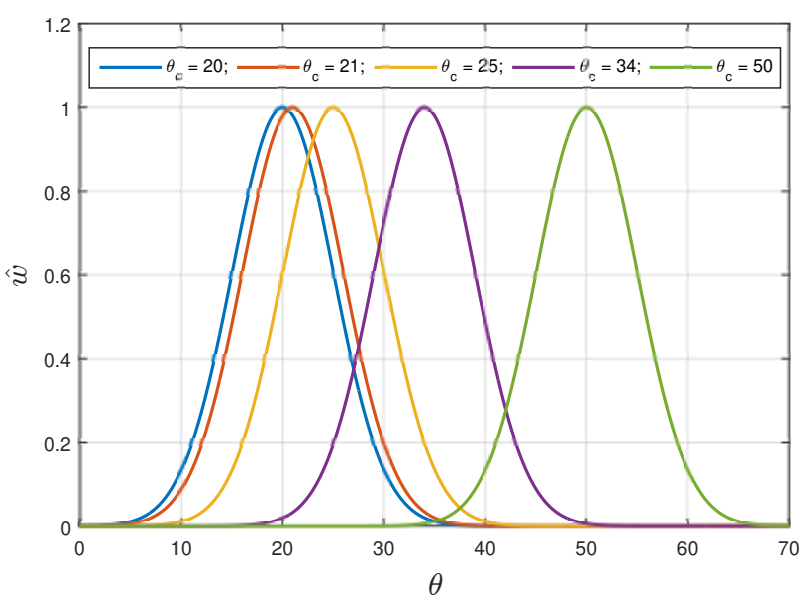

(a) Efeito de $\theta_{c}$ em $\hat{w}$

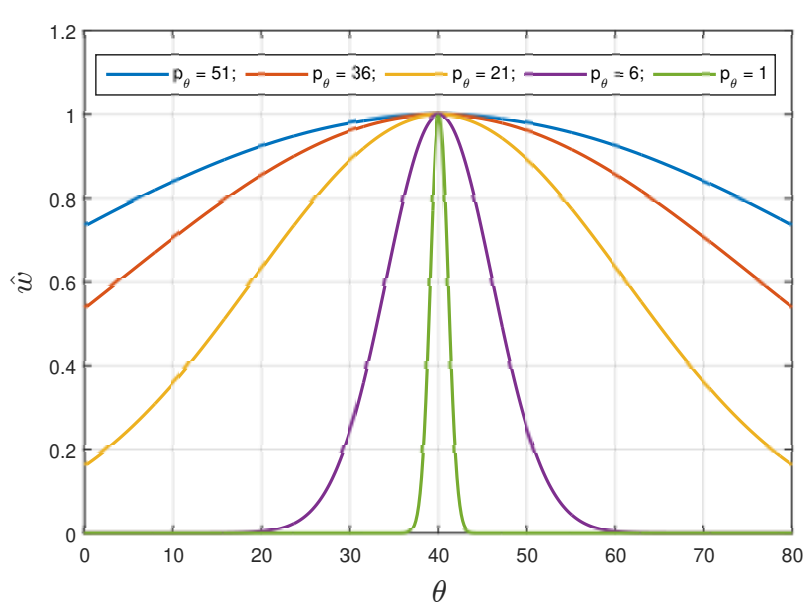

(b) Efeito de $p_{\theta}$ em $\hat{w}$

Figura 3.11 - Efeitos causados pela alteração de $\theta_{c}$ e $p_{\theta}$ na função de distribuição normal $\hat{w}$

evitar estas instabilidades e termina com um valor $p_{\theta}=p_{\theta}^{\min }$. No trabalho de (KIYONO; SILVA; REDDY, 2017), $p_{\theta}^{\text {min }}$ é determinado empiricamente. Porém, é possível determinar este valor em função do menor número normalizado positivo do tipo float, que depende da arquitetura da máquina utilizada (ZURAS et al., 2008). Para um computador de 64 bits, este valor denominado realmin equivale a $2,2251 \cdot 10^{-308}$.

Considerando um indesejável caso particular (KIYONO; SILVA; REDDY, 2017) onde o valor de $\theta$ está exatamente entre o menor intervalo $\Delta \theta_{c}^{\text {min }}$ entre todos os ângulos candidatos, como demonstrado na Fig. 3.12, onde $\Delta \theta_{c}^{\text {min }}=\left|\theta_{c}^{i+1}-\theta_{c}^{i}\right|$, é possível notar que para $\theta=\frac{\theta_{c}^{i+1}+\theta_{c}^{i}}{2}$, o valor de $\hat{w}$ tende a 0 . A partir disto, pode-se estabelecer a Eq. (3.14)

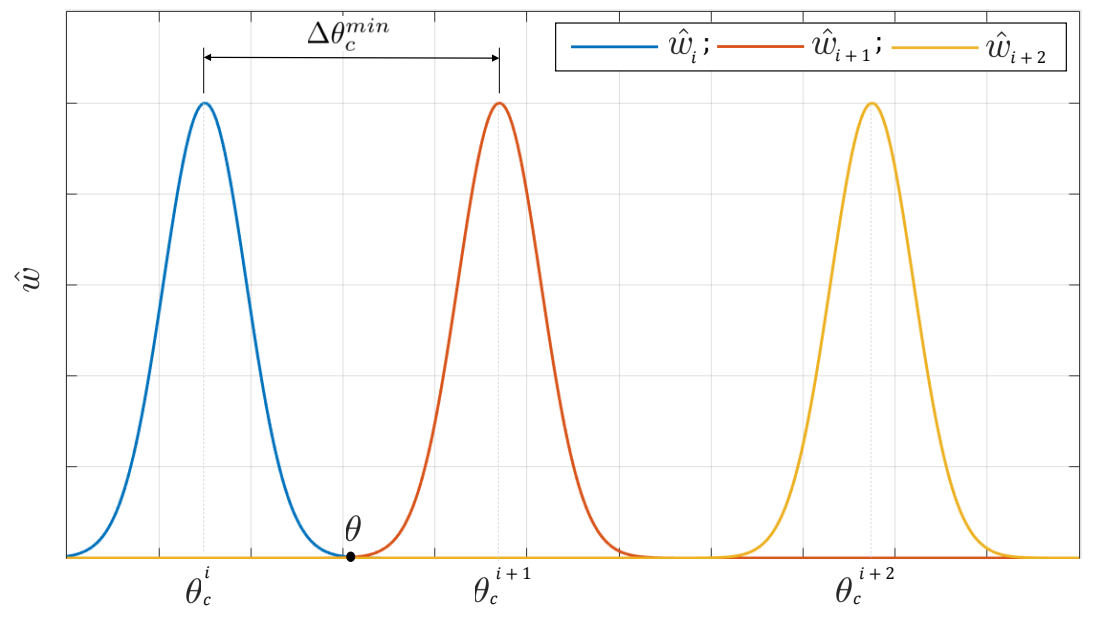

Figura 3.12 - $\theta$ exatamente entre o menor intervalo entre os ângulos candidatos 


$$
\hat{w}=e^{-\frac{\left(\frac{\theta_{c}^{i+1}-\theta_{c}^{i}}{2}\right)}{2 p_{\theta}^{2}}}=\text { realmin }
$$

Partindo da Eq. (3.14) é possível determinar um valor de $p_{\theta}^{\min }$, o qual representa o menor valor de $p_{\theta}$, que pode ser representado com precisão float. Caso $p_{\theta}<p_{\theta}^{\min }$, erros numéricos podem ser introduzidos ou valores indeterminados para $\hat{w}$ podem ser obtidos em função de uma divisão por zero (Not A Number ou NAN em linguagens como Python e Matlab). Portanto, $p_{\theta}^{\min }$ é calculado como

$$
p_{\theta}^{\min }=\left(-\frac{\left(0.5\left(\theta_{c}^{i+1}-\theta_{c}^{i}\right)\right)^{2}}{2 \ln (\text { realmin })}\right)^{\frac{1}{2}}
$$

\subsubsection{Modelo de Interpolação Autopenalizável da Orientação da Fibra (MIAFIB)}

Como citado em Seção 1.1, a principal característica do MIAFIB é a definição das funções seno e cosseno por meio de séries de Taylor (ver Eq. (3.16)).

$$
\begin{gathered}
\sin (\theta)=\sum_{n=0}^{\infty} \frac{(-1)^{n}}{(2 n+1) !} \theta^{2 n+1} \\
\cos (\theta)=\sum_{n=0}^{\infty} \frac{(-1)^{n}}{(2 n) !} \theta^{2 n}
\end{gathered}
$$

onde $\theta=\{\theta \mid-\pi<\theta<\pi\}$ representa o ângulo das fibras. Os extremos do intervalo são excluídos pelo fato de que constituem múltiplos mínimos locais das funções seno e cosseno e, por consequência, podem acarretar em um mínimo local da função objetivo no problema de otimização (STEGMANN; LUND, 2005; SALAS et al., 2018). Com o intuito de evitar instabilidades numéricas, (SALAS et al., 2018) propõe uma parametrização na variável $\theta$ em relação a $\pi$ (ver Eq. (3.17))

$$
\theta=\pi \hat{\theta}, \quad-1<\hat{\theta}<1
$$

A variável $\hat{\theta}$ é denominada pseudo-orientação e é a variável de projeto do modelo MIAFIB. A determinação dos extremos do intervalo correspondente a $\hat{\theta}$ é realizada de acordo com a precisão deseja no ângulo das fibras, definida pela Eq. (3.18)

$$
\hat{\theta}=\frac{\left\lfloor\theta \cdot 10^{b}+0.5\right\rfloor}{180 \cdot 10^{b}}
$$


onde a variável $\theta$ é representada em graus, $b$ é o número de casas decimais desejadas para $\theta$ e $\lfloor\cdot\rfloor$ corresponde à função piso. Salas et al. (2018) utiliza $b=0$, o que equivale a definir $\theta$ como $\theta=\left\{\theta: \theta \in \mathbb{Z} \mid-179^{\circ} \leq \theta \leq 179^{\circ}\right\}$.

As funções seno e cosseno do MIAFIB, baseadas na Eq. (3.16) são definidas de acordo com Eq. (3.19)

$$
\begin{gathered}
s=\sin (\theta)=\sum_{p_{f}=0}^{p_{f}^{\max }} \frac{(-1)^{p_{f}}}{\left(2 p_{f}+1\right) !}(\pi \hat{\theta})^{2 p_{f}+1} \\
c=\cos (\theta)=\sum_{p_{f}=0}^{p_{f}^{\max }} \frac{(-1)^{p_{f}}}{\left(2 p_{f}\right) !}(\pi \hat{\theta})^{2 p_{f}}
\end{gathered}
$$

onde $p_{f}$ é o penalizador do MIAFIB que pode assumir valores entre 0 e $p_{f}^{\max }$.

Nas primeiras iterações do problema de otimização utilizam-se poucos termos nas séries de Taylor apresentadas na Eq. (3.19), (geralmente 1 ou 2). Com o progresso das iterações mais termos são adicionados, até que $p_{f}$ alcance o valor $p_{f}=p_{f}^{\max }$. Por essa razão, o modelo é denominado autopenalizável, o acréscimo de mais termos nas séries de Taylor equivale a um método de continuação, o que torna possível contornar os mínimos locais do problema (SALAS et al., 2018).

A Fig. 3.13 demonstra os valores das aproximações para as funções seno e cosseno com $\hat{\theta}$ variando de -1.5 a 1.5. Nota-se por meio da Fig. $3.13 a$ que quando $p_{f}=0$, a aproximação por séries de Taylor da função seno resulta em uma curva linear, enquanto que para o mesmo valor de $p_{f}$, a aproximação para a função cosseno possui um valor constante (ver Fig. 3.13b). Conforme o valor do penalizador $p_{f}$ aumenta, mais mínimos locais são adicionados às séries, o que acontece de forma gradativa. Por fim, por meio da Fig. 3.13 podemos concluir que quando $p_{f}=16$, tem-se uma boa aproximação em relação aos valores reais de seno e cosseno.

\subsection{Restrição de continuidade}

A configuração de uma estrutura otimizada obtida por OT pode apresentar descontinuidades nas fibras (ver Fig. 3.14). Isto deve ser evitado, pois uma mudança abrupta na direção pode representar uma região onde haverá concentração de tensão mecânica, além de representar uma dificuldade a mais na manufatura. Outro problema a ser evitado é o acúmulo de fibras em uma mesma região (ver Fig. 3.15), uma vez que regiões onde as fibras se acumulam podem representar imperfeições, pois podem ser interpretadas como regiões onde não há a presença de matriz. A descontinuidade das fibras, neste trabalho, é tratada utilizando-se um filtro baseado na equação de Helmholtz modificada (LAZAROV; 


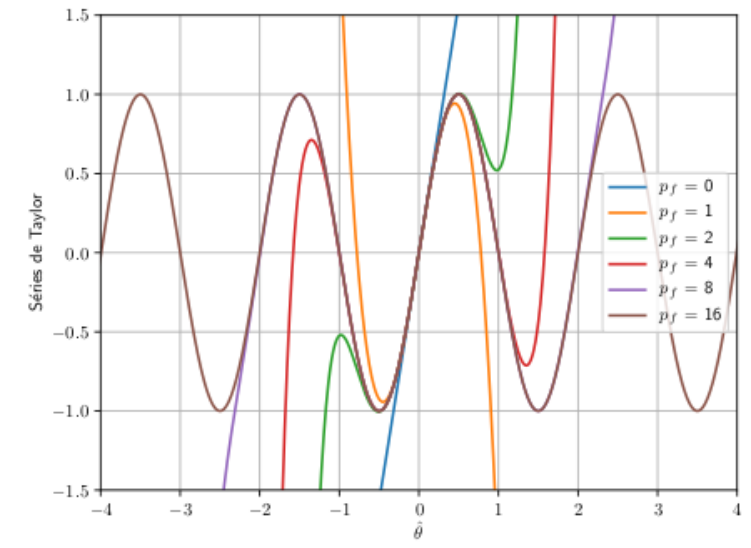

(a) Aproximação da função seno.

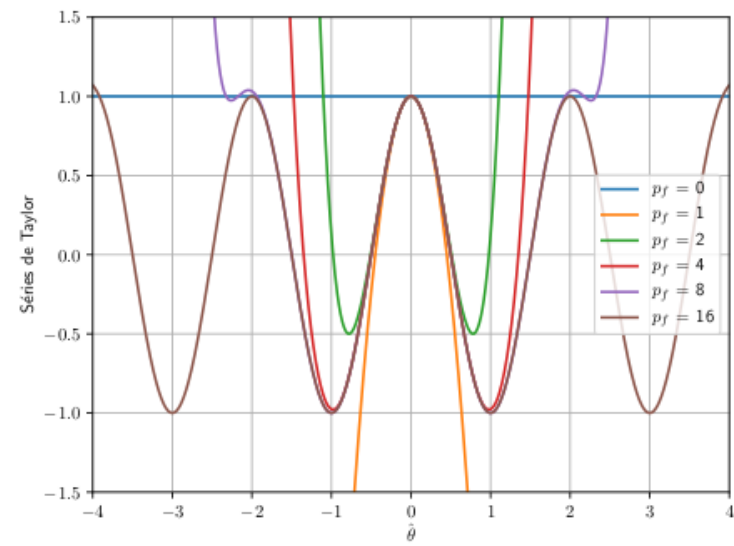

(b) Aproximação da função cosseno.

Figura 3.13 - Aproximação da funções seno e cosseno por séries de Taylor.

SIGMUND, 2011) apresentado na Seção 3.4 e com o acréscimo de uma restrição, baseada no modelo de Borrvall (ver (BORRVALL; PETERSSON, 2003),Seção 3.6.3) no problema de otimização. A restrição além de garantir a continuidade das fibras, também trata o problema de acúmulo das mesmas.
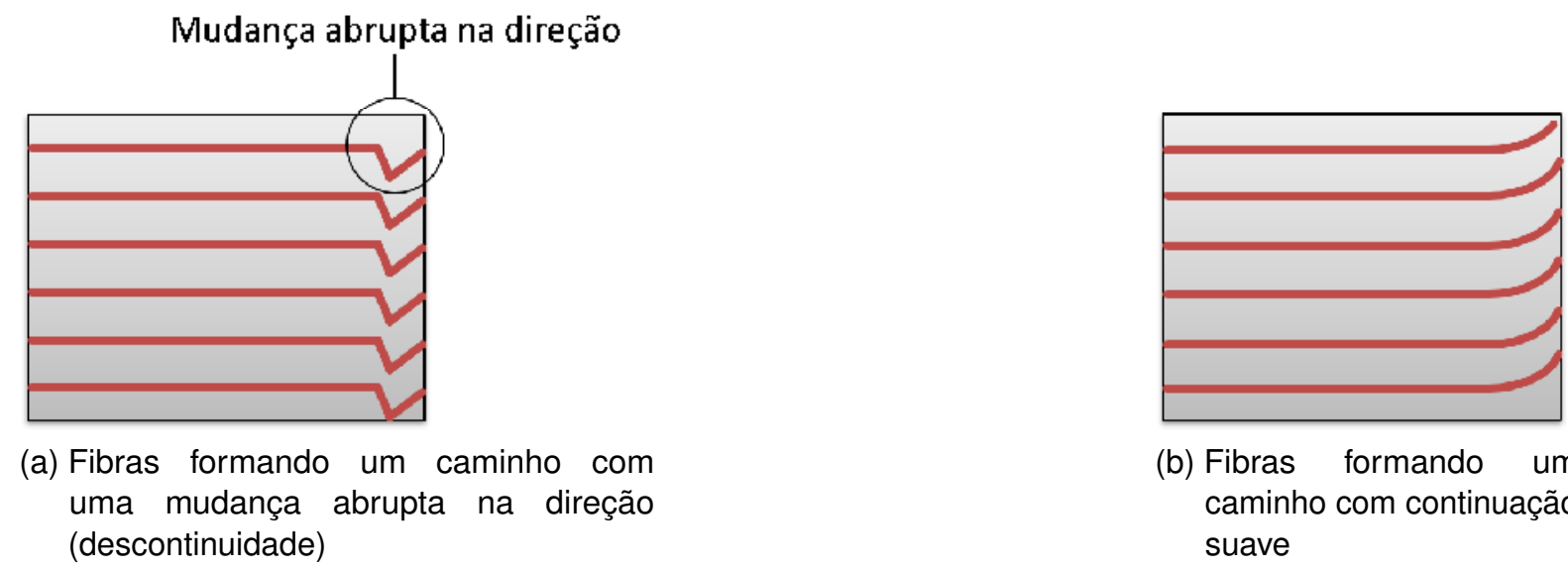

(b) Fibras formando um caminho com continuação suave

Figura 3.14 - Continuidade das fibras

\subsubsection{Equação de Stokes}

A importância da continuidade das fibras foi introduzida na Seção 1.4, onde os problemas relacionados a dificuldade de manufatura e concentração de tensões foram citados. Uma das soluções propostas neste trabalho para tratar o problema de descontinuidade e o acúmulo das fibras é baseada em uma analogia com o escoamento interno de um fluido. Um exemplo de escoamento é apresentado na Fig. 3.16, onde as curvas azuis são linhas de corrente que tangenciam os vetores de velocidades. Percebe-se, para o exemplo ilustrado na Fig. 3.16, que as linhas de corrente não apresentam mudanças abruptas em suas direções 


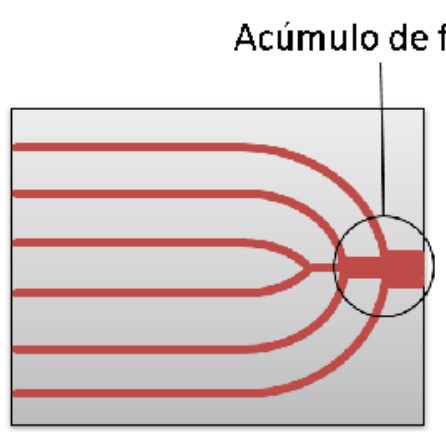

(a) Fibras se acumulando em um região do domínio.

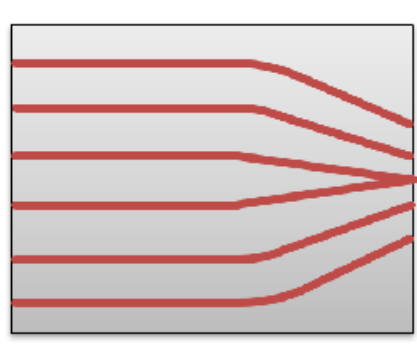

(b) Fibras formando um caminho sem acúmulo de fibras.

Figura 3.15 - Acúmulo de fibras

e que as mesmas não acumulam como na ilustração apresentada na Fig. 3.15a. Valendo-se destas características, desenvolve-se uma restrição de continuidade que compara o ângulo das fibras com um campo de velocidades obtido por meio do escoamento laminar de um fluido, onde o volume de controle é definido pela distribuição da pseudo-densidade $\bar{\rho}$ obtida através da OT.

Para tanto, utiliza-se uma equação constitutiva em termos de pressão e velocidade, considerando o fluxo de um fluido Newtoniano, incompressível e com viscosidade dinâmica $\mu_{l}$ constante, escoando em um volume de controle $V_{l}$, como demonstrado na Eq. (3.20) (BORRVALL; PETERSSON, 2003)

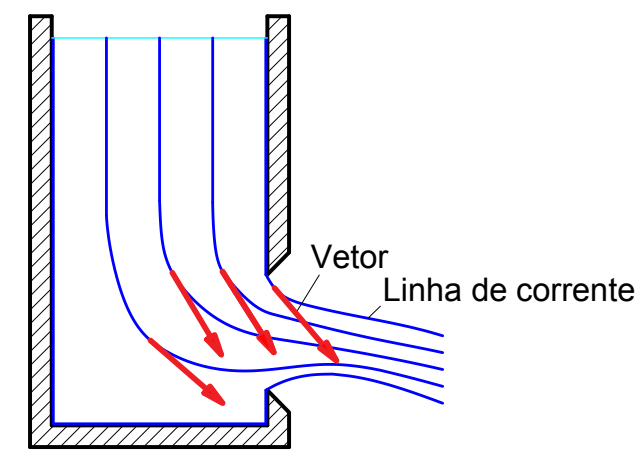

Figura 3.16 - Escoamento em um tanque aberto.

$$
\begin{gathered}
\boldsymbol{\sigma}_{l}=-\bar{p} \mathbf{I}+2 \mu_{d} \mathbf{D}\left(\overline{\mathbf{v}}_{l}\right) \\
\mathbf{D}\left(\overline{\mathbf{v}}_{l}\right)=\frac{1}{2}\left(\nabla \overline{\mathbf{v}}_{l}+\left(\nabla \overline{\mathbf{v}}_{l}\right)^{T}\right)
\end{gathered}
$$

onde $\sigma_{l}$ é o tensor de tensões para o fluido, $\bar{p}$ é a pressão, $\mathbf{D}\left(\overline{\mathbf{v}}_{l}\right)$ é o tensor da taxa de deformação e $\overline{\mathbf{v}}_{l}$ é a velocidade. A equação de movimento é definida como (BORRVALL; 
PETERSSON, 2003)

$$
\rho_{0}\left(\frac{\partial \overline{\mathbf{v}}_{l}}{\partial t}+\left(\nabla \overline{\mathbf{v}}_{l}\right) \overline{\mathbf{v}}_{l}\right)=\nabla \cdot \boldsymbol{\sigma}_{l}+\mathbf{f}_{l}^{B}
$$

onde $\rho_{0}$ e $\mathbf{f}_{l}^{B}$ são a densidade e força de volume do fluido respectivamente. Assumindo as hipóteses de que o fluxo do fluido é lento e está em regime permanente, a viscosidade $\mu_{l}$ é constante e o fluido é incompressível, a partir das Eqs. (3.20a), (3.20b) e (3.21) e da condição de incompressibilidade, é possível definir as equações de Stokes como sendo (BORRVALL; PETERSSON, 2003)

$$
\begin{gathered}
\mu_{l} \nabla^{2} \overline{\mathbf{v}}_{l}=\nabla \bar{p}-\mathbf{f}_{l}^{B} \\
\nabla \cdot \overline{\mathbf{v}}_{l}=0
\end{gathered}
$$

Multiplicando a Eq. (3.22a) por uma função de teste de velocidade $\delta \overline{\mathbf{v}}_{l}$, a qual possui valores iguais a zero nas fronteiras $S_{l}$ do volume de controle, integrando sobre $V_{l}$ e utilizando o teorema de Green, obtêm-se a equação da Potência Virtual (ver Eq. (3.23)) (BORRVALL; PETERSSON, 2003)

$$
\mu_{l} \int_{V_{l}} \nabla \overline{\mathbf{v}}_{l} \cdot \nabla \delta \overline{\mathbf{v}}_{l} d V_{l}-\int_{V_{l}} \bar{p} \nabla \cdot \delta \overline{\mathbf{v}}_{l} d V_{l}=\int_{V_{l}} \mathbf{f}_{l}^{B} \cdot \delta \overline{\mathbf{v}}_{l} d V_{l}
$$

O mesmo procedimento é aplicado para a Eq. (3.22b), multiplicando-a por uma função de teste de pressão $\delta \bar{p}$ e integrando sobre $V_{l}$ (ver Eq. (3.24))

$$
-\int_{V_{l}} \delta \bar{p} \nabla \cdot \overline{\mathbf{v}}_{l} d V_{l}=0
$$

\subsubsection{Modelo de Borrvall}

As Eqs. (3.23) e (3.24) constituem a Forma Fraca do Problema de Stokes, que consiste na determinação da velocidade $\overline{\mathbf{v}}$ e da pressão $\bar{p}$. Considerando a hipótese de que o fluxo ocorre entre duas superfícies próximas e não planas, separadas por uma distância dada por uma função $\rho_{l}(x)$, pode-se escrever o problema de Stokes como sendo (BORRVALL; 
PETERSSON, 2003)

$$
\begin{array}{r}
\int_{V_{l}} \alpha\left(\rho_{l}\right) \overline{\mathbf{v}}_{l} \cdot \delta \overline{\mathbf{v}}_{l} d V+\mu_{l} \int_{V_{l}} \nabla \overline{\mathbf{v}}_{l} \cdot \nabla \delta \overline{\mathbf{v}}_{l} d V_{l}-\int_{V_{l}} \bar{p} \nabla \cdot \delta \overline{\mathbf{v}}_{l} d V_{l}-\int_{V_{l}} \delta \bar{p} \nabla \cdot \overline{\mathbf{v}}_{l} d V_{l}= \\
=\int_{V_{l}} \mathbf{f}_{l}^{B} \cdot \delta \overline{\mathbf{v}}_{l} d V_{l}
\end{array}
$$

O primeiro termo acrescido na Eq. (3.25) é um termo de absorção, que equivale a potência dissipada por cisalhamentos que ocorrem fora do plano, o qual é função de $\alpha\left(\rho_{l}\right)$, que pode ser interpretada como o inverso da permeabilidade, ou seja, as regiões do domínio onde $\alpha\left(\rho_{l}\right)>>1$ representam sólido enquanto regiões do domínio onde $\alpha\left(\rho_{l}\right)=0$ representam fluido. Para isso, utiliza-se a função de interpolação, parametrizada por um penalizador $q$, apresentada em Eq. (3.26) (BORRVALL; PETERSSON, 2003).

$$
\alpha_{q}\left(\rho_{l}\right)=\bar{\alpha}+(\underline{\alpha}-\bar{\alpha}) \rho_{l} \frac{1+q}{\rho_{l}+q}
$$

onde $\underline{\alpha}$ e $\bar{\alpha}$ são os valores máximo e mínimo para a função de interpolação $\alpha$. A Fig. 3.17 demonstra a influência da alteração do parâmetro $q$ na função de interpolação $\alpha\left(\rho_{l}\right)$. É possível observar que quanto maior o valor de $q$, mais linear torna-se a função de interpolação.

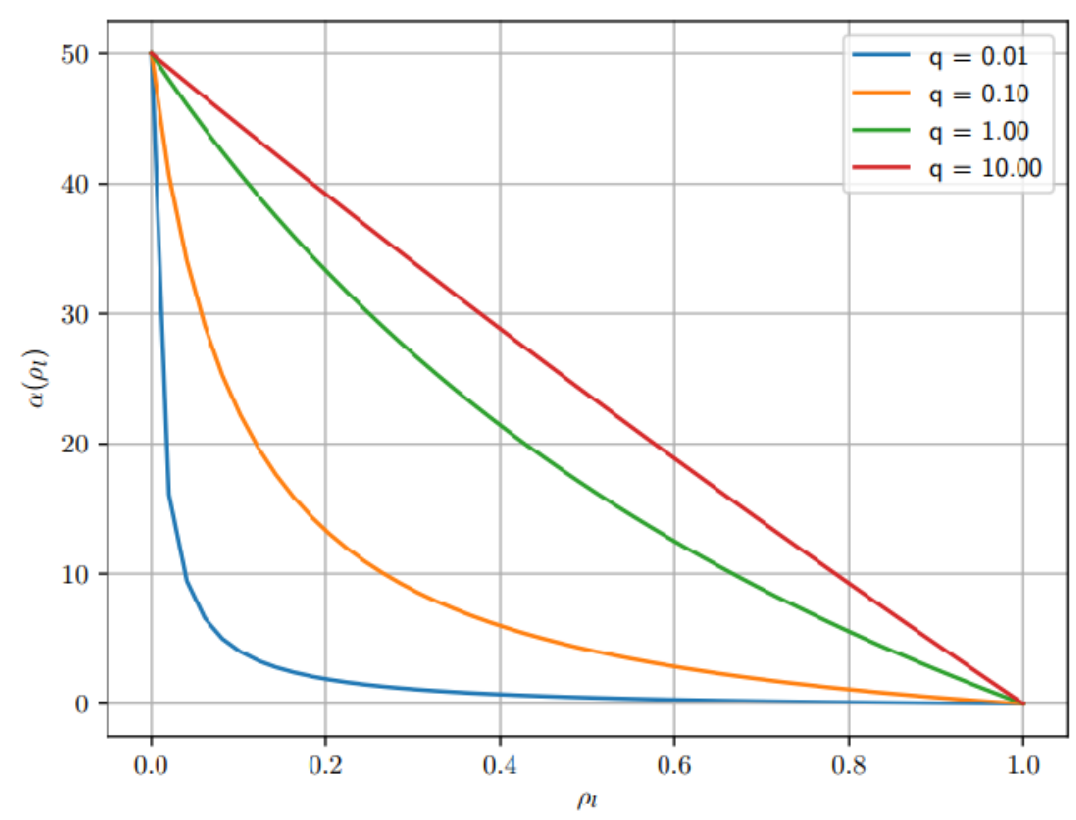

Figura 3.17 - Influência do penalizador $q$ na função de interpolação $\alpha\left(\rho_{l}\right)$

A implementação da Eq. (3.25) é realizada no software FEniCS. Para a implementação em outras linguagens de programação, utilizando formulações matricial e vetorial, consultar o trabalho de Borrvall e Petersson (2003). 


\subsubsection{Restrição baseada no modelo de Borrvall}

Neste trabalho, propõem-se uma restrição baseada no modelo de Borrvall para tratar os problemas de descontinuidade e acúmulo das fibras, em que o campo de velocidades, obtido por meio deste modelo, é utilizado como restrição para os ângulos. Considerando $\boldsymbol{\theta}$ um vetor que possui como componentes as projeções do ângulo de uma fibra nas direções $x$ e $y$ (ver Eq. (3.27)) e $\mathbf{v}_{l}$ o vetor de velocidade obtido por meio da solução da Eq. (3.25), sabe-se que se o produto vetorial entre esses dois vetores é igual a $\mathbf{0}$, os mesmos são paralelos entre si.

$$
\boldsymbol{\theta}=\left[\begin{array}{c}
\cos (\theta) \\
\operatorname{sen}(\theta)
\end{array}\right]
$$

Pode-se então, definir uma restrição de desigualdade, onde o produto vetorial entre $\boldsymbol{\theta}$ e $\mathbf{v}_{l}$ deve ser menor ou igual a um vetor $\beta$ (ver Eq. (3.28))

$$
\begin{aligned}
\boldsymbol{\theta} \times \mathbf{v}_{l} & \leq \boldsymbol{\beta} \\
\boldsymbol{\beta} & =\left[\begin{array}{l}
0 \\
0 \\
\beta
\end{array}\right]
\end{aligned}
$$

onde $\beta$ é um escalar que representa a magnitude do produto vetorial entre $\theta$ e $\mathbf{v}_{l}$, o qual pode ser interpretado como uma medida que representa o paralelismo entre estes dois vetores, sendo que quanto menor o valor de $\|\beta\|$, maior o paralelismo.

A restrição deve ser aplicada em todo o domínio $V$. Para isso, faz-se o produto interno $\boldsymbol{\beta} \cdot \boldsymbol{\beta}$ e integra-se no domínio $V$ (ver Eq. (3.29))

$$
\int_{V}\left[\left(\boldsymbol{\theta} \times \mathbf{v}_{l}\right) \cdot\left(\boldsymbol{\theta} \times \mathbf{v}_{l}\right)-\beta^{2}\right] d V \leq 0
$$

A Eq. (3.29) é implementada no software Dolfin Adjoint e utilizada como restrição para tratar a descontinuidade e acúmulo das fibras. 


\section{FORMULAÇÃO DO PROBLEMA DE OT PARA O PROJETO DE MATERIAIS COMPÓSITOS REFORÇADOS COM FIBRA}

O objetivo deste trabalho é definir uma formulação de OT para determinar a orientação da fibra e ao mesmo tempo a distribuição de material que maximize a rigidez de um certo domínio fixo, impondo restrições de volume. A definição do problema é ilustrada na Fig. 4.1, onde são apresentadas as condições de contorno e uma representação do domínio final otimizado.

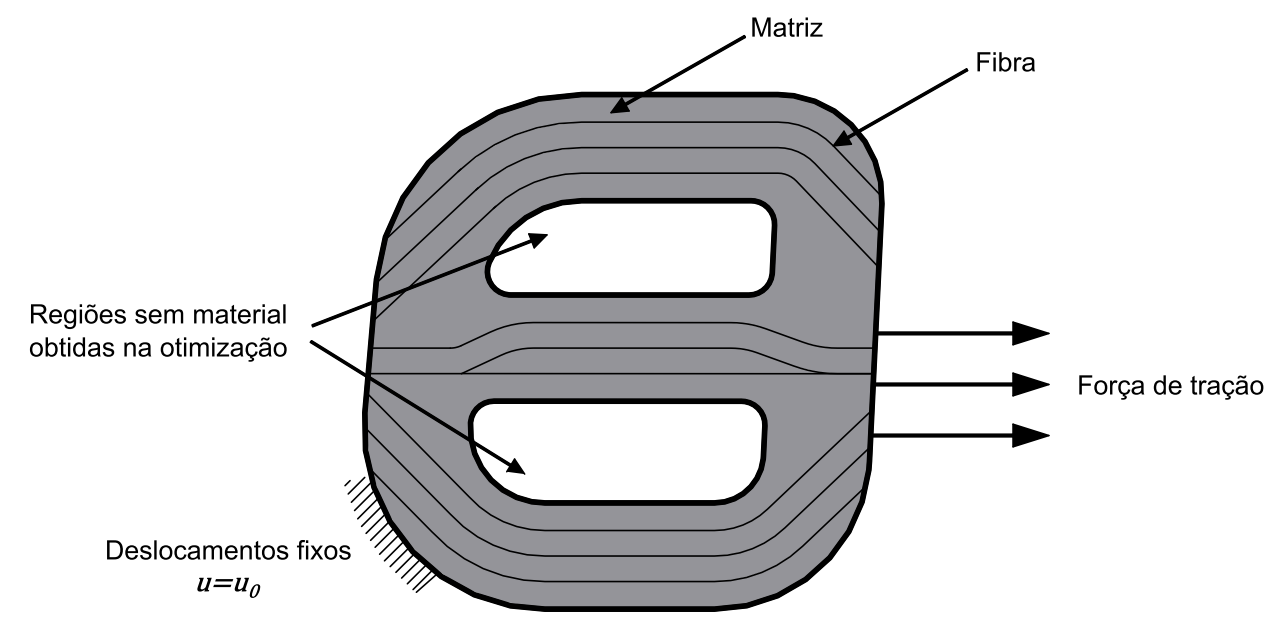

Figura 4.1 - Definição do problema de maximização de rigidez em um material compósito utilizando OT

\subsection{Formulação do problema para maximização da rigidez}

Maximizar a rigidez de uma estrutura equivale a minimizar sua flexibilidade. Portanto, objetivo do problema de otimização é minimizar o trabalho realizado pelas forças externas (ver Eq. (4.1))

$$
\underset{\mathbf{u}, \theta}{\operatorname{minimizar}} \quad W_{e x t}=\int_{V} \mathbf{u}^{T} \mathbf{f}^{B} d V+\int_{S_{f}} \mathbf{u}^{S_{f}^{T}} \mathbf{f}^{S_{f}} d S+\sum_{i} \mathbf{u}^{i^{T}} \mathbf{r}_{C}^{i}
$$


A Eq. (2.33) estabelece que os trabalhos virtuais realizados pelas forças externas e internas devem ser iguais, portanto

$$
\delta W_{e x t}=\delta W_{i n t}=\int_{V} \delta \varepsilon^{T} \boldsymbol{\sigma} d V
$$

O problema de otimização pode então ser escrito como

$$
\begin{aligned}
\underset{\mathbf{u}, \theta, \rho}{\operatorname{minimizar}} \quad J & =W_{\text {ext }} \\
\text { tal que } \quad F_{1} & =\delta W_{\text {ext }}-\delta W_{\text {int }}=0 \quad \forall \delta \mathbf{u} \in V \\
F_{2} & =\int_{V} \mathbf{r}^{2} \nabla \tilde{\mathbf{a}} \cdot \nabla v d V+\int_{V} \tilde{\mathbf{a}} \cdot v d V-\int_{V} \boldsymbol{a} \cdot v d V=0 \\
F_{3} & =\int_{V} \bar{\rho}(\mathbf{x}) d V-V \leq 0 \\
F_{4} & =\int_{V}\left[\left(\boldsymbol{\theta} \times \mathbf{v}_{l}\right) \cdot\left(\boldsymbol{\theta} \times \mathbf{v}_{l}\right)-\beta^{2}\right] d V \leq 0 \\
F_{5} & =\bar{\rho}_{\min }-\bar{\rho}(\mathbf{x}) \leq 0 \\
F_{6} & =\bar{\rho}(\mathbf{x})-1 \leq 0 \\
F_{7} & =\theta_{\min }-\theta(\mathbf{x}) \leq 0 \\
F_{8} & =\theta(\mathbf{x})-\theta_{\max } \leq 0
\end{aligned}
$$

onde $J$ é o trabalho virtual externo, $F_{1}$ é a equação do princípio dos trabalhos virtuais, $F_{2}$ é a equação modificada de Helmholtz (ver Eq. (3.8), $F_{3}$ é a restrição de volume, $F_{4}$ é a restrição de continuidade (ver Eq. (3.29), $F_{5}$ e $F_{6}$ são restrições de caixa para a pseudo-densidade $\bar{\rho}$ e $F_{7}$ e $F_{8}$ são restrições de caixa para o ângulo da fibra $\theta$. Os valores de $\theta_{\min }$ e $\theta_{\max }$ variam de acordo com o modelo utilizado. Na equação $F_{2}$ as variáveis a e $\mathbf{r}$ são definidas de acordo com as Eqs. (4.4) e (4.5)

$$
\begin{gathered}
\boldsymbol{a}=\left[\begin{array}{l}
\theta(\mathbf{x}) \\
\bar{\rho}(\mathbf{x})
\end{array}\right] \\
\mathbf{r}=\left[\begin{array}{cc}
r_{\theta} & 0 \\
0 & r_{\bar{\rho}}
\end{array}\right]
\end{gathered}
$$

\subsection{Sensibilidades}

Os gradientes da função objetivo e restrições, também denominadas sensibilidades, nos permitem avaliar como alterações nas variáveis de projeto interferem no comportamento da estrutura. Além disto, as sensibilidades são necessárias para a implementação de algoritmo de otimização de primeira e segunda ordem. Existem diferentes abordagens para 
a determinação das sensibilidades. Uma forma de obtê-las é escrevendo o Lagrangiano do problema, igualando sua variação a zero e isolando os termos arbitrários. O Lagrangiano do problema expresso na Eq. (4.3) é dado por

$$
\begin{aligned}
L(u(\tilde{\theta}(\theta), \tilde{\rho}(\bar{\rho}), \theta, \bar{\rho}), \tilde{\theta}(\theta), \tilde{\rho}(\bar{\rho}), \theta, \bar{\rho}) & = \\
& =J(u(\tilde{\theta}(\theta), \tilde{\rho}(\bar{\rho}), \theta, \bar{\rho}), \tilde{\theta}(\theta), \tilde{\rho}(\bar{\rho}), \theta, \bar{\rho})+ \\
& -\lambda_{1} F_{1}(u(\tilde{\theta}(\theta), \tilde{\rho}(\bar{\rho}), \theta, \bar{\rho}), \tilde{\theta}(\theta), \tilde{\rho}(\bar{\rho}), \theta, \bar{\rho})+ \\
& -\lambda_{2} F_{2}(\tilde{\theta}(\theta), \tilde{\rho}(\bar{\rho}), \theta, \bar{\rho})+ \\
& +\lambda_{3} F_{3}(\bar{\rho})+\lambda_{4} F_{4}(\theta)+\lambda_{5} F_{5}(\bar{\rho})+ \\
& +\lambda_{6} F_{6}(\bar{\rho})+\lambda_{7} F_{7}(\theta)+\lambda_{8} F_{8}(\theta)
\end{aligned}
$$

A variação do Lagrangiano é representada por $\delta L$ (ver Eq. (4.7)), onde $\delta$ é denominado Operador Variacional.

$$
\begin{aligned}
& \delta L(u(\tilde{\theta}(\theta), \tilde{\rho}(\bar{\rho}), \theta, \bar{\rho}), \tilde{\theta}(\theta), \tilde{\rho}(\bar{\rho}), \theta, \bar{\rho})= \\
& =\int_{V}\left(\frac{\partial L}{\partial u} \delta u+\frac{\partial L}{\partial \tilde{\theta}} \delta \tilde{\theta}+\frac{\partial L}{\partial \tilde{\rho}} \delta \tilde{\rho}+\frac{\partial L}{\partial \theta} \delta \theta+\frac{\partial L}{\partial \rho} \delta \bar{\rho}\right) d V=0
\end{aligned}
$$

As variações $\delta[\cdot]$ são por definição arbitrárias e em função disto pode-se concluir a partir da variação $\delta \mathbf{u}$ que

$$
\frac{\partial J}{\partial \mathbf{u}}-\lambda_{1} \frac{F_{1}}{\partial \mathbf{u}}=0
$$

A Eq. (4.8) é denominada Equação Adjunta, pois a partir dela é possível determinar o valor da variável adjunta $\lambda_{1}$. A arbitrariedade das variações $\delta \tilde{\theta}$ e $\delta \tilde{\rho}$, juntamente com a Eq. (4.8), permite que as Eqs. (4.9) e (4.10) sejam definidas

$$
\begin{aligned}
& \frac{\partial J}{\partial \tilde{\theta}}-\lambda_{1} \frac{\partial F_{1}}{\partial \tilde{\theta}}-\lambda_{2} \frac{\partial F_{2}}{\partial \tilde{\theta}}=0 \\
& \frac{\partial J}{\partial \tilde{\rho}}-\lambda_{1} \frac{\partial F_{1}}{\partial \tilde{\rho}}-\lambda_{2} \frac{\partial F_{2}}{\partial \tilde{\rho}}=0
\end{aligned}
$$

As Eqs. (4.9) e (4.10) também são equações adjuntas, pois por meio delas, é possível determinar o valor da variável $\lambda_{2}$. Por fim, a sensibilidade da função objetivo $J$ em relação as variáveis de projeto é determinada utilizando a arbitrariedade das variações $\delta \theta$ e $\delta \bar{\rho}$ e as 
equações Eqs. (4.8) a (4.10) (ver Eqs. (4.11) e (4.12) )

$$
\begin{aligned}
& \frac{\partial J}{\partial \theta}=\lambda_{1} \frac{\partial F_{1}}{\partial \theta}+\lambda_{2} \frac{\partial F_{2}}{\partial \theta}-\lambda_{4} \frac{\partial F_{4}}{\partial \theta}-\lambda_{7} \frac{\partial F_{7}}{\partial \theta}-\lambda_{8} \frac{\partial F_{8}}{\partial \theta} \\
& \frac{\partial J}{\partial \bar{\rho}}=\lambda_{1} \frac{\partial F_{1}}{\partial \bar{\rho}}+\lambda_{2} \frac{\partial F_{2}}{\partial \bar{\rho}}-\lambda_{3} \frac{\partial F_{3}}{\partial \bar{\rho}}-\lambda_{5} \frac{\partial F_{5}}{\partial \bar{\rho}}-\lambda_{6} \frac{\partial F_{6}}{\partial \bar{\rho}}
\end{aligned}
$$

onde $\lambda_{k}, \quad(k=1, \ldots, 7)$ devem respeitar as condições de otimalidade, também conhecidas como condições KKT (mais detalhes sobre as condições KKT podem ser encontrados em (KUHN; TUCKER, 1951; ARORA, 2004; NOCEDAL; WRIGHT, 2006; BAZARAA; SHERALI; SHETTY, 2013)). A abordagem apresentada nas Eqs. (4.6) a (4.12) é chamada de Método Adjunto, conhecido por ser eficiente em casos onde o número de variáveis de projeto é maior que o número de restrições.

As sensibilidades também podem ser determinadas a partir do problema na forma discretizada, esta abordagem é mais comumente encontrada na literatura. A seguir, apresenta-se uma revisão que demonstra como os autores (LAZAROV; SIGMUND, 2011; KIYONO; SILVA; REDDY, 2017; SALAS et al., 2018) determinaram as sensibilidades da função objetivo $J$ em relação as variáveis de projeto de cada um dos modelos. (LAZAROV; SIGMUND, 2011) calcula a sensibilidade da função objetivo utilizando o método adjunto a partir do problema discretizado. A derivada parcial da função objetivo em relação a variável de projeto filtrada é calculada por meio de

$$
\begin{gathered}
\frac{\partial J}{\partial \hat{\mathbf{a}}}=\mathbf{s}_{f}=\sum_{m}-p \int_{V^{(m)}} \mathbf{N}^{(m)} \tilde{\rho}^{(m)^{p-1}} \mathbf{u}^{(m)} \tilde{\mathbf{B}}^{T} \overline{\mathbf{C}}^{0} \tilde{\mathbf{B}} \mathbf{u}^{(m)} d V^{(m)} \\
\tilde{\mathbf{B}}=\tilde{\nabla} \mathbf{N}
\end{gathered}
$$

onde $\tilde{\nabla}$ é o operador elástico linear, $\mathbf{N}$ é a matriz de funções de interpolação para o problema linear elástico. A Eq. (B.2), apresentada no Apêndice B, pode ser escrita como (LAZAROV; SIGMUND, 2011)

$$
\begin{gathered}
\mathbf{K}_{f} \hat{\mathbf{a}}=\mathbf{Z} \mathbf{a} \\
\mathbf{p}_{f}=\mathbf{Z} \mathbf{a}
\end{gathered}
$$

onde $\mathbf{Z}$ é uma matriz cujas linhas são composta pela integral $\int_{V^{(m)}} \mathbf{N}^{(m)^{T}} d V^{(m)}$ e possui dimensões mxn. $m$ equivale ao número de graus de liberdade no campo filtrado e $n$ ao 
número de células. A sensibilidade da variável filtrada em relação a variável de projeto é definida na Eq. (4.17) (LAZAROV; SIGMUND, 2011).

$$
\frac{\partial \tilde{\mathbf{a}}}{\partial \mathbf{a}}=\mathbf{Z}^{T} \mathbf{K}_{h}^{-1}
$$

Por fim, a sensibilidade da função objetivo em relação a variável de projeto pode ser escrita como (LAZAROV; SIGMUND, 2011)

$$
\frac{\partial J}{\partial \mathbf{a}}=\mathbf{Z}^{T} \mathbf{K}_{h}^{-1} \mathbf{s}_{f}
$$

(KIYONO; SILVA; REDDY, 2017) apresentam a sensibilidade da função objetivo $J$ em relação ao ângulo $\theta$ no modelo OFDN como demonstrado na Eq. (4.19)

$$
\frac{\partial J}{\partial \theta^{(m)}}=-\hat{\mathbf{u}} \frac{\partial \mathbf{K}}{\partial \theta^{(m)}} \hat{\mathbf{u}}
$$

A sensibilidade para um problema de maximização de rigidez em uma estrutura considerando apenas uma variável de projeto em regime linear é bem conhecido na literatura. O desenvolvimento desta equação pode ser visto em (BENDSøE; SIGMUND, 2003). Como cada elemento na formulação do modelo OFDN possui uma variável de projeto, a derivada parcial da matriz K em relação a $\theta$ presente do lado direito da equação Eq. (4.19) pode ser determinada por elemento (ver Eq. (4.20)) (KIYONO; SILVA; REDDY, 2017)

$$
\frac{\partial \mathbf{K}^{(m)}}{\partial \theta^{(m)}}=\int_{V^{(m)}} \mathbf{B}^{T} \frac{\partial \overline{\mathbf{C}}_{e}}{\partial \theta^{(m)}} \mathbf{B} d V^{(m)}
$$

Partindo da definição do tensor constitutivo efetivo $\overline{\mathbf{C}}_{e}$ dado pela Eq. (3.9), pode-se determinar a sua derivada parcial em relação ao ângulo $\theta$ de acordo com a Eq. (4.21) (KIYONO; SILVA; REDDY, 2017)

$$
\frac{\partial \overline{\mathbf{C}}_{e}}{\partial \theta^{(m)}}=\sum_{i=1}^{n_{c}} \frac{\partial w_{i}}{\partial \theta^{(m)}} \mathbf{C}_{\mathbf{i}}
$$

As derivadas parciais das funções de ponderação $w_{i}$ e de distribuição normal $\hat{w}_{i}$ em relação a $\theta$ são demonstradas nas Eqs. (4.22) e (4.23)

$$
\frac{\partial w_{i}}{\partial \theta^{(m)}}=\frac{1}{\left(\sum_{k=1}^{n_{c}} \hat{w}_{k}\right)^{2}}\left(\frac{\partial \hat{w}_{i}}{\partial \theta^{(m)}} \sum_{k=1}^{n_{c}} \hat{w}_{k}-\hat{w}_{i} \sum_{k=1}^{n_{c}} \frac{\partial \hat{w}_{k}}{\partial \theta^{(m)}}\right)
$$




$$
\frac{\partial \hat{w}_{i}}{\partial \theta^{(m)}}=-\frac{\theta^{(m)}-\theta_{c}^{i}}{p_{\theta}^{2}} e^{-\frac{\left.\left(\theta^{(m)}\right) \theta_{c}^{i}\right)^{2}}{2 p_{\theta}^{2}}}
$$

Para o modelo MIAFIB, as sensibilidades da função objetivo em relação a variável de pseudo-orientação $\hat{\theta}$ são determinadas por meio da equação Eq. (4.24)

$$
\frac{\partial J}{\partial \hat{\theta}}=-\hat{\mathbf{u}} \frac{\partial \mathbf{K}}{\partial \hat{\theta}} \hat{\mathbf{u}}
$$

O modelo MIAFIB, assim como o modelo OFDN, utiliza uma variável de projeto por elemento, portanto, a definição da derivada parcial da matriz de rigidez $\mathbf{K}$ pode seguir o mesmo princípio adotado na Eq. (4.20)

$$
\frac{\partial \mathbf{K}^{(m)}}{\partial \hat{\theta}^{(m)}}=\int_{V^{(m)}} \mathbf{B}^{T} \frac{\partial \overline{\mathbf{C}}_{e}}{\partial \hat{\theta}^{(m)}} \mathbf{B} d V^{(m)}
$$

A derivada do tensor constitutivo $\overline{\mathbf{C}}$ em relação a pseudo-orientação $\hat{\theta}$ é definida na Eq. (4.26)

$$
\frac{\partial \overline{\mathbf{C}}}{\partial \hat{\theta}}=\frac{\partial \mathbf{T}^{-1}}{\partial \hat{\theta}} \mathbf{C} \mathbf{R} \mathbf{T} \mathbf{R}^{-1}+\mathbf{T}^{-1} \mathbf{C} \mathbf{R} \frac{\partial \mathbf{T}}{\partial \hat{\theta}} \mathbf{R}^{-1}
$$

onde as derivadas da matriz de transformação $\mathbf{T}$ e da sua inversa $\mathbf{T}^{-1}$ são definidas para nas Eqs. (4.27) e (4.28)

$$
\begin{gathered}
\frac{\partial \mathbf{T}}{\partial \hat{\theta}}=\pi\left[\begin{array}{cccccc}
-s_{2} & s_{2} & 0 & 0 & 0 & 2 c_{2} \\
s_{2} & -s_{2} & 0 & 0 & 0 & -2 c_{2} \\
0 & 0 & 0 & 0 & 0 & 0 \\
0 & 0 & 0 & -s & -c & 0 \\
0 & 0 & 0 & c & -s & 0 \\
-c_{2} & c_{2} & 0 & 0 & 0 & -2 s_{2}
\end{array}\right] \\
\frac{\partial \mathbf{T}^{-1}}{\partial \hat{\theta}}=\pi\left[\begin{array}{cccccc}
-s_{2} & s_{2} & 0 & 0 & 0 & -2 c_{2} \\
s_{2} & -s_{2} & 0 & 0 & 0 & 2 c_{2} \\
0 & 0 & 0 & 0 & 0 & 0 \\
0 & 0 & 0 & -s & c & 0 \\
0 & 0 & 0 & -c & -s & 0 \\
c_{2} & -c_{2} & 0 & 0 & 0 & -2 s_{2}
\end{array}\right]
\end{gathered}
$$


e as matrizes de transformação para o caso de EPT são definidas nas Eqs. (4.29) e (4.30)

$$
\begin{gathered}
\frac{\partial \mathbf{T}_{E P T}}{\partial \hat{\theta}}=\pi\left[\begin{array}{ccc}
-s_{2} & s_{2} & 2 c_{2} \\
s_{2} & -s_{2} & -2 c_{2} \\
-c_{2} & c_{2} & -2 s_{2}
\end{array}\right] \\
\frac{\partial \mathbf{T}_{E P T}^{-1}}{\partial \hat{\theta}}=\pi\left[\begin{array}{ccc}
-s_{2} & s_{2} & -2 c_{2} \\
s_{2} & -s_{2} & 2 c_{2} \\
c_{2} & -c_{2} & -2 s_{2}
\end{array}\right]
\end{gathered}
$$

onde as notações $s_{2}$ e $c_{2}$ representam o $\sin (2 \theta)$ e $\cos (2 \theta)$ respectivamente.

Neste trabalho, em função da facilidade de implementação, as sensibilidades e equações adjuntas são determinadas por meio de um Algoritmo de Diferenciação Automática implementado na biblioteca libadjoint. Esta abordagem necessita da definição de um sistema de equações diferenciais parciais $F(u ; m)=0$ e um funcional de interesse $J(u(m), m)$, em que, $u$ é a solução do sistema de equações e $m$ é uma variável de projeto ou controle. Por meio da regra da cadeia, pode-se calcular a derivada do funcional $J$ em relação a $m$ (ver Eq. (4.31)) (FUNKE, 2013)

$$
\frac{d J}{d m}=\frac{\partial J}{\partial u} \frac{d u}{d m}+\frac{\partial J}{\partial m}
$$

A derivada da solução $u$ em relação a variável de projeto $m$ pode ser obtida derivando-se o sistema de equações diferenciais parciais (ver Eq. (4.32))

$$
\frac{\partial F}{\partial u} \frac{d u}{d m}+\frac{\partial F}{\partial m}=0 \Longrightarrow \frac{d u}{d m}=-\frac{\partial F^{-1}}{\partial u} \frac{\partial F}{\partial m}
$$

Substituindo Eq. (4.32) em Eq. (4.31), obtêm-se o gradiente do funcional com termos que podem ser derivados explicitamente (ver Eq. (4.33)) (FUNKE, 2013)

$$
\frac{d J}{d m}=-\lambda^{*} \frac{\partial F}{\partial m}+\frac{\partial J}{\partial m}
$$

onde $\lambda^{*}$ é o vetor adjunto (ver Eq. (4.34)) e o asterisco representa o conjugado transposto.

$$
\lambda^{*}=\frac{\partial J}{\partial u} \frac{\partial F^{-1}}{\partial u}
$$


O problema adjunto é então definido como (FUNKE, 2013):

$$
\frac{\partial F^{*}}{\partial u} \lambda=\frac{\partial J^{*}}{\partial u}
$$

A sensibilidade do funcional $J$ é obtido determinando-se a solução da equação Eq. (4.34), substituindo esta solução na Eq. (4.33) e por fim, calculando seu valor (FUNKE, 2013).

O funcional de interesse e o sistema de equações diferenciais parciais no caso do problema de otimização representado em Eq. (4.3) são a função objetivo $J$ e as equações $F_{i}$ respectivamente. A solução $u$ equivale aos deslocamentos u e a variável de projeto $m$ pode ser um vetor $\mathbf{m}$ que contempla a pseudo-densidade $\bar{\rho}$ e o ângulo da fibra $\theta$. 


\section{IMPLEMENTAÇÃO NUMÉRICA}

O problema de OT apresentado no Capítulo 4 é resolvido por meio de um processo iterativo que envolve a solução da equação do princípio dos trabalhos virtuais com uma distribuição inicial para as variáveis de projeto (ver Seção 2.4), a solução da equação adjunta (ver Seção 4.2), o cálculo das sensibilidades da função objetivo e das restrições ver (Seção 4.2), a atualização das variáveis de projeto por meio de um algoritmo de otimização e a verificação do critério de parada.

O critério de parada pode ser um número máximo de iterações ou uma verificação na convergência do resultado de otimização por meio da comparação de duas iterações consecutivas. Essa verificação pode ser realizada por meio da norma entre duas iterações consecutivas da variável a (ver Eq. (4.4))

$$
\left\|\mathbf{a}_{k}-\mathbf{a}_{k-1}\right\|<=\text { tol }
$$

onde tol pode variar de acordo com o problema resolvido. Geralmente tol $<1 \cdot 10^{3}$.

Neste trabalho a implementação é realizada por meio do software FEniCS, um software livre que tem como principal característica o fato de gerar automaticamente códigos para solucionar de forma eficiente equações diferenciais por meio do MEF. Este software utiliza o compilador FFC para gerar códigos "Unified Form-Assembly Code" (UFC) em C++ a partir de uma descrição matemática de alto nível UFL das equações diferenciais em sua forma fraca (ver Fig. 5.1). Essas informações são utilizadas pela biblioteca DOLFIN, que funciona como principal interface do FEniCS e serve também para combinar as funcionalidades dos outros componentes (SKAVHAUG, 2016; LANGTANGEN; LOGG, 2017; SÁ, 2016).

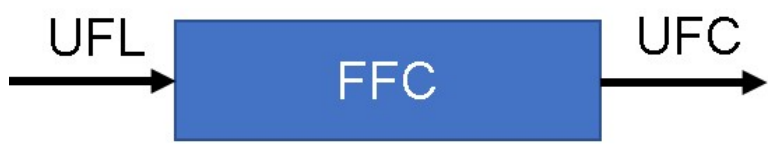

Figura 5.1 - Funcionamento do compilador FFC. Adaptado de (SKAVHAUG, 2016)

\subsection{Implementação do sistema adjunto}

O código para implementação do sistema adjunto pode ser determinado a partir da equação de estado de diferentes maneiras. A partir da equação de estado em sua forma contínua, é possível definir as equações adjuntas também em sua forma contínua, o que geralmente é feito por meio de diferenciação simbólica ou sem o auxílio de programas de 
computador. Após este passo, pode-se discretizar estas equações para, por fim, realizar a implementação. Uma outra alternativa é utilizar ferramentas de diferenciação automática a partir da equação de estado já implementada. Um terceiro modo possível, consiste em obter a forma discreta da equação de estado e, a partir dela, determinar as equações adjuntas já em sua forma discreta e após isto, realizar sua implementação. Isso é possível utilizando-se a biblioteca libadjoint, que permite a derivação e solução do sistema adjunto de forma automática. O libadjoint define a representação simbólica da equação de estado e, a partir disto, determina a representação simbólica do sistema de equações adjuntas. $A$ biblioteca Dolfin Adjoint realiza a interação entre o DOLFIN e o libadjoint, interpretando o modelo adjunto e traduzindo-o para a mesma linguagem de alto nível da equação de estado (FUNKE; FARRELL, 2013; SÁ, 2016).

Em função da facilidade de implementação, é utilizado neste trabalho a sequência destacada na Fig. 5.2, ou seja, utilizando o pacote UFL obtêm-se a equação de estado discretizada, por meio da biblioteca libadjoint o sistema de equações adjuntas é obtido, e por fim, o código adjunto é compilado por meio do pacote FFC (SÁ, 2016).

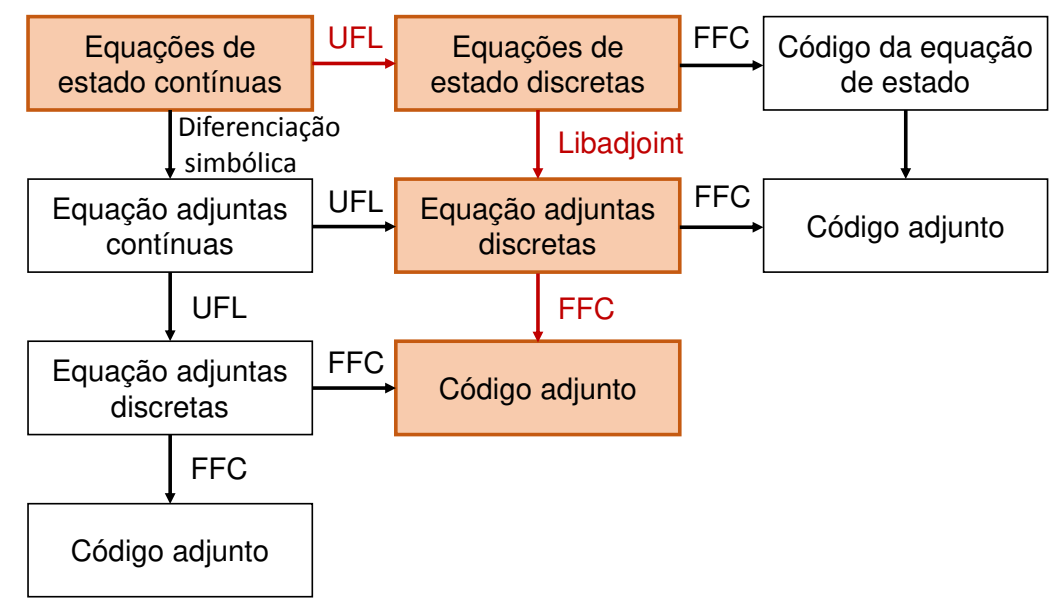

Figura 5.2 - Diferentes modos para obtenção do código para implementação do sistema de equações adjuntas. Adaptado de (FUNKE, 2013; SÁ, 2016)

\subsection{Método do ponto interior}

A fase seguinte do processo iterativo da OT consiste na atualização das variáveis de projeto, a qual deve ser realizada por meio de um algoritmo de otimização. Diversos algoritmo de otimização podem ser encontrados na literatura, entre os quais estão o Critério de Otimalidade (TAYLOR; ROSSOW, 1977; SAVE et al., 1985; ROZVANY, 2012; ROZVANY, 2014), o Método das Assíntotas Móveis (SVANBERG, 1987) e a Programação Linear 
Sequencial (GRIFFITH; STEWART, 1961; BUZBY, 1974; BODDINGTON; RANDALL, 1979). No entanto, neste trabalho o algoritmo utilizado é o Método do Ponto Interior, também conhecido como Método da Barreira. O método da barreira está implementado no pacote "Interior Point Optimizer" (IPOpt) que possui uma série de métodos para a obtenção de um melhor desempenho, como por exemplo um filtro de busca em linha, além de facilitar a implementação, pois o pacote pode ser importado para o ambiente FEniCS onde seus parâmetros podem ser controlados. No pacote IPOpt um método da barreira primal-dual é utilizado para solucionar um problema de otimização não linear formulado como

$$
\begin{array}{cl}
\underset{x \in \mathbb{R}}{\operatorname{minimizar}} & f(x) \\
\text { tal que } & c_{i}(x)=0 \\
& x \geq 0
\end{array}
$$

Para o problema definido em Eq. (5.2) a função de barreira mais utilizada é a Função de Barreira Logarítmica (ver Eq. (5.3)) (NOCEDAL; WRIGHT, 2006)

$$
P(x)=\sum_{i} \log c_{i}(x)
$$

O método da barreira determinada soluções para uma sequência de problemas de barreira definidos de acordo com Eq. (5.4) (NOCEDAL; WRIGHT, 2006; ARORA, 2004; WÄCHTER; BIEGLER, 2006).

$$
\begin{array}{cl}
\underset{x \in \mathbb{R}}{\operatorname{minimizar}} & \phi_{\mu}(x, \mu)=f(x)-\mu P(x) \\
\text { tal que } & c_{i}(x)=0
\end{array}
$$

onde $\phi(x, \mu)$ é a combinação da função objetivo e da função de barreira logarítmica e $\mu$ é o parâmetro de barreira. O parâmetro $\mu$ deve ser positivo e como pode ser visto na Eq. (5.4), determina quão importante é a função $P(x)$ no resultado de otimização da função $\phi(x, \mu)$. 0 algoritmo determina a solução para o problema Eq. (5.4) para um valor de $\mu$ fixo. Após isto, o valor de $\mu$ é reduzido e a solução para o próximo problema de barreira é determinado a partir da solução prévia. Maiores detalhes relacionados ao funcionamento do pacote IPOpt podem ser encontrados em (WÄCHTER; BIEGLER, 2006).

\subsection{Implementação da OT}

A implementação da OT consiste primeiramente em definir no ambiente FEniCS um domínio fixo e as condições de contorno. Depois disto, são fornecidos valores iniciais para $\theta$ e $\bar{\rho}$. Com essas informações, a equação de estado é solucionada. Com os valores do 
deslocamento u determinados, o valor e as sensibilidades da função objetivo são calculados. A partir destes dados, uma nova configuração para $\theta$ e $\bar{\rho}$ é definida no domínio por meio do método da barreira implementado no pacote IPOpt. O critério de parada é avaliado para determinar se o processo pode ser encerrado ou se é necessário iniciar uma nova iteração. Este processo é demonstrado no fluxograma exibido na Fig. 5.3

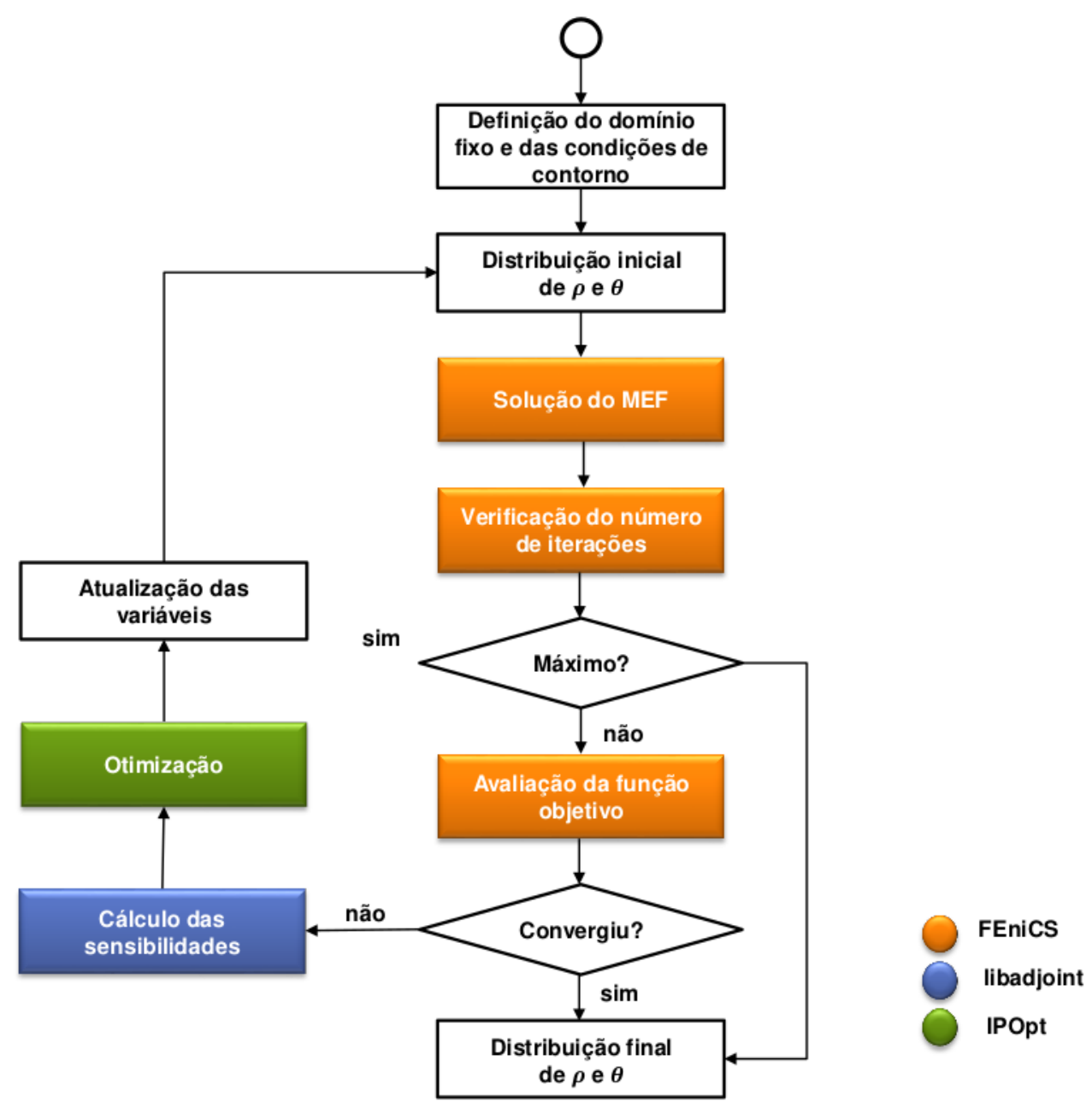

Figura 5.3 - Fluxograma de implementação de OT 


\section{RESULTADOS}

Neste capítulo são apresentados resultados obtidos para a maximização da rigidez em uma estrutura compósita reforçada com fibras. Na Seção 6.1, são apresentados resultados para o modelo OFDN-m considerando somente a orientação das fibras. Diferentes parâmetros $r$ são testados no filtro de Helmholtz para que seu efeito seja verificado no resultado final. A restrição de continuidade não é utilizada nos exemplos desta seção.

Na Seção 6.2 são apresentados resultados obtidos utilizando o modelo MIAFIB para distribuição de material no domínio e orientação das fibras. A restrição de continuidade é utilizada para garantir a continuidade e tratar o problema de acumulo de fibras.

\subsection{Resultados de OT aplicada a orientação de fibras}

Nesta seção são apresentados resultados de otimização para compósitos reforçados com fibras considerando somente a orientação das fibras. São realizadas otimizações de três diferentes domínios. O material considerado em todos os exemplos da Seção 6.1 é o epoxy com fibras de vidro (KAW, 2005). Suas propriedades são apresentadas na Tabela 6.1. As regiões representadas por blocos cinzas equivalem à áreas totalmente fixadas, ou seja, nas fronteiras do domínio em contato com blocos da cor cinza, a condição de contorno de Dirichlet é $\mathbf{u}=\mathbf{0}$. As forças aplicadas garantem que a simulação permaneça no regime linear elástico, obedecendo as hipóteses anteriormente adotadas. Nestas simulações, utiliza-se uma malha regular composta por elementos tetraédricos com interpolação linear, tanto para a determinação dos deslocamentos, quanto para a determinação dos ângulos das fibras (ver Fig. 6.1 ). Para os dois primeiros exemplos (Seções 6.1.1 e 6.1.2) as dimensões dos elementos são $5 \mathrm{~mm} \times 5 \mathrm{~mm} \times 5 \mathrm{~mm}$, enquanto que para o terceiro resultado (Seção 6.1.3) as dimensões são $2.5 \mathrm{~mm} \times 2.5 \mathrm{~mm} \times 2.5 \mathrm{~mm}$. Os ângulos candidatos considerados são $[-90,-78.75,-67.5,-56.25,-45,-33.75,-22.5,-11.25,0, \ldots$ sim. , 90] medidos em graus e apenas forças de superfície são consideradas.

\begin{tabular}{ccccccccc}
\hline$E_{1}[G P a]$ & $E_{2}[G P a]$ & $E_{3}[G P a]$ & $G_{12}[G P a]$ & $G_{31}[G P a]$ & $G_{23}[G P a]$ & $v_{12}$ & $v_{13}$ & $v_{23}$ \\
\hline 38,6 & 8,27 & 8,27 & 4,14 & 4,14 & 2,58 & 0,26 & 0,26 & 0,60 \\
\hline
\end{tabular}

Tabela 6.1 - Propriedades do material para resultados da Seção 6.1. 

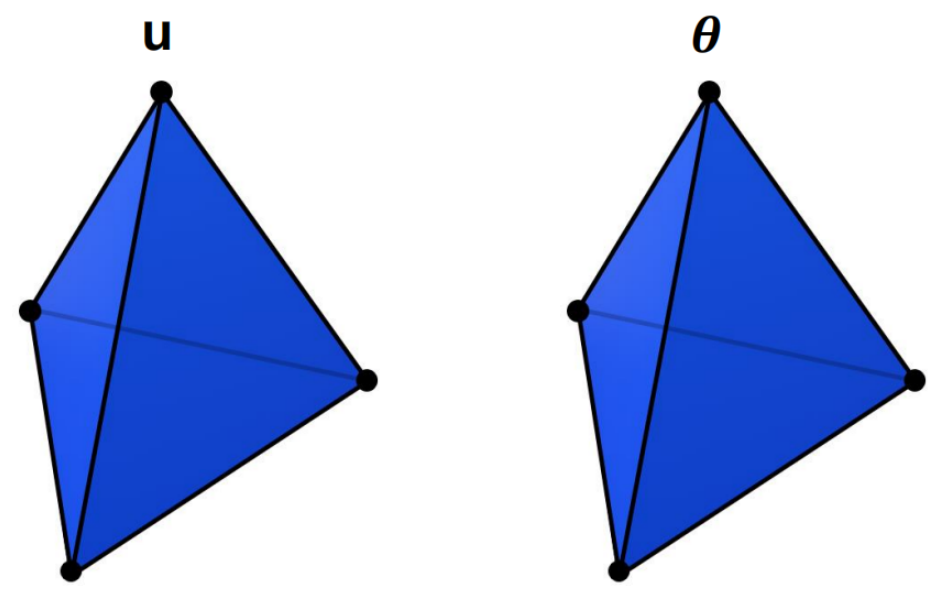

Figura 6.1 - Elementos tetraédricos com interpolação linear.

\subsubsection{Viga engastada}

O primeiro exemplo numérico consiste em uma viga engastada com carga distribuída $F=15 \mathrm{kN} / \mathrm{m}^{2}$ aplicada em sua extremidade. Neste exemplo solucionado com o modelo OFDN-m, o penalizador $p_{\theta}$ vale inicialmente 20 e a cada dez iterações seu valor decresce uma unidade até atingir o valor $p_{\theta}=4$. Enquanto $p_{\theta}$ possuir valores entre 4 e 0.4 , seu valor diminui em 0.1 a cada duas iterações. Por fim, a cada duas iterações, o valor de $p_{\theta}$ diminui 0.05 até que seu valor atinja 0.3. O número máximo de iterações é igual a 1000. A Fig. 6.2a apresenta as dimensões em $\mathrm{mm}$ e as condições de contorno. A Fig. 6.2b demonstra um resultado em representação tridimensional com fibras longas.

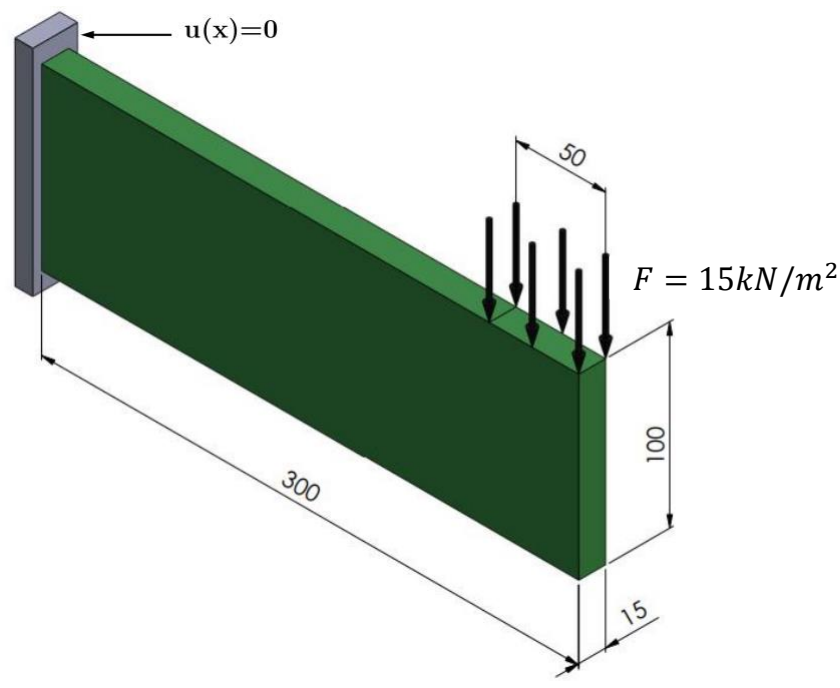

(a) Domínio da viga engastada.

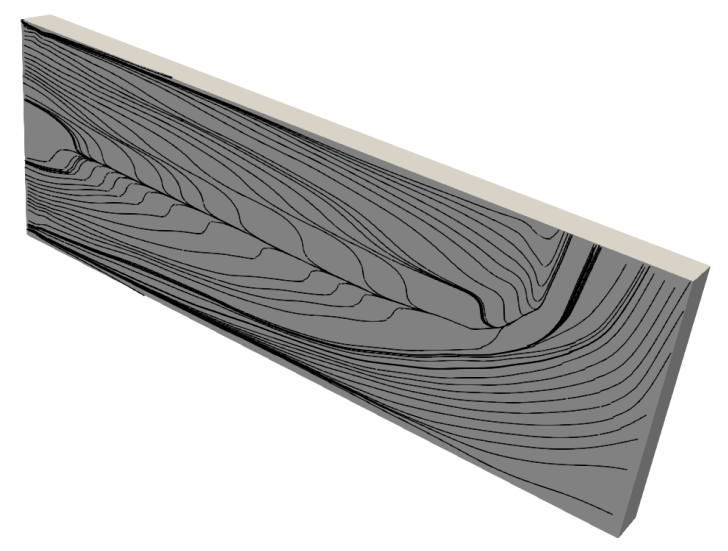

(b) Representação de um resultado tridimensional.

Figura 6.2 - Viga engastada.

Os resultados são apresentados de forma semelhante aos resultados encontrados na literatura, ou seja, uma fibra reta de pequeno comprimento é representada em cada nó 
da malha de elementos finitos. A Fig. 6.8 demonstra os resultados com duas distribuições iniciais diferentes, $\theta=0^{\circ}$ (Figs. 6.3a, 6.3c e 6.3e) e $\theta=45^{\circ}$ (Figs. 6.3b, 6.3d e 6.3f), para os parâmetros $r$ do filtro de Helmholtz iguais a $5 \mathrm{~mm}, 15 \mathrm{~mm}$ e $30 \mathrm{~mm}$.

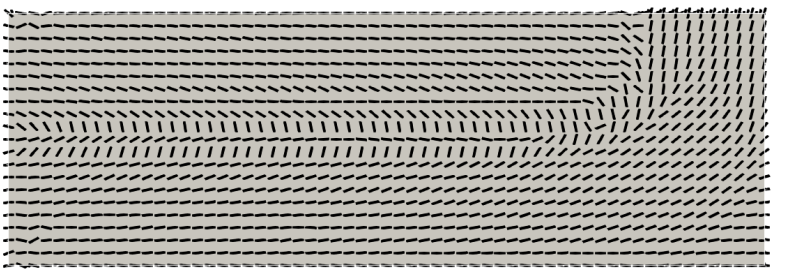

(a) Resultado do modelo OFDN-m para $\theta_{0}=0^{\circ} \mathrm{e} r=$ (b) Resultado do modelo OFDN-m para $\theta_{0}=45^{\circ} \mathrm{e}$ $5 \mathrm{~mm}$

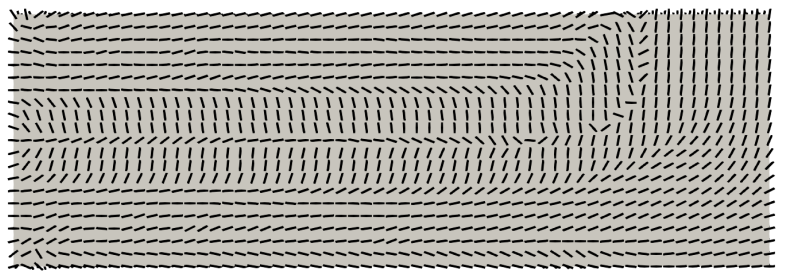

(c) Resultado do modelo OFDN-m para $\theta_{0}=0^{\circ}$ e $r=(d)$ $15 \mathrm{~mm}$

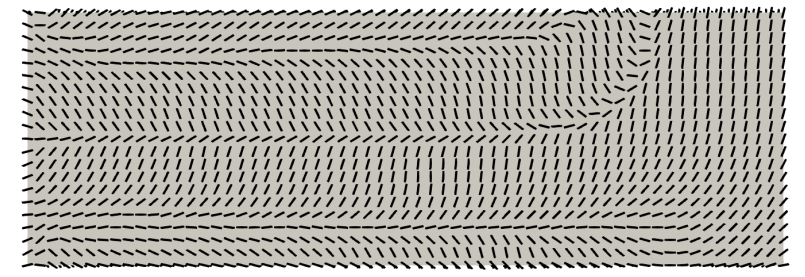

(e) Resultado do modelo OFDN-m para $\theta_{0}=0^{\circ}$ e $r=(1)$ $30 \mathrm{~mm}$

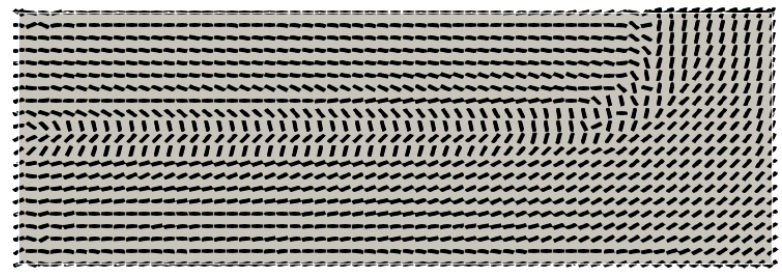

$r=5 \mathrm{~mm}$

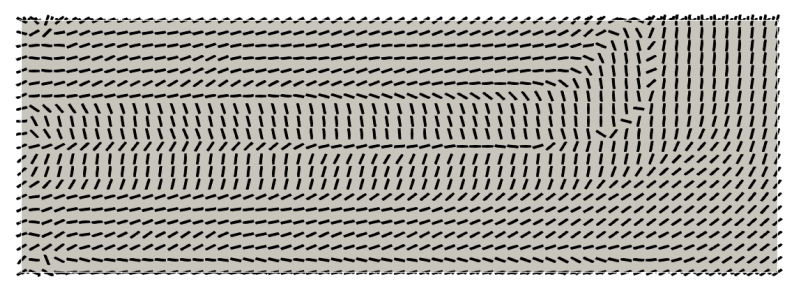

(d) Resultado do modelo OFDN-m para $\theta_{0}=45^{\circ} \mathrm{e}$ $r=15 \mathrm{~mm}$

Figura 6.3 - Orientação otimizada das fibras para uma viga engastada sujeita a uma carga de $F=15 \mathrm{kN} / \mathrm{m}^{2}$ obtida com o modelos OFDN-m.

Os resultados apresentados na Fig. 6.3 demonstram que as fibras se alinham à direção da aplicação da força, na área abaixo onde esta é aplicada, para minimizar o deslocamento naquela região maximizando assim a rigidez. Nas outras regiões do domínio, as fibras se alinham de forma a impedir a flexão da viga, o que fica mais evidente para os casos onde $r=5 \mathrm{~mm}$ (ver Figs. 6.3a e 6.3b).

O efeito do filtro de Helmholtz no resultado otimizado pode ser observado por meio da comparação entre as Figs. 6.3a a 6.3f. Nas Figs. 6.3a e 6.3b pode-se observar 3 fileiras horizontais, que se iniciam do lado esquerdo e estão localizadas aproximadamente no centro do domínio $(y \approx 50 \mathrm{~mm})$, onde as fibras possuem ângulos próximos a $-90^{\circ} \mathrm{e} 90^{\circ}$. Ao redor dessas 4 fileiras e em algumas regiões no interior das mesmas, é possível notar alterações abruptas nas direções das fibras. As Figs. 6.3c e 6.3d apresentam um número maior de fileiras horizontais com ângulos próximos aos candidatos $-90^{\circ}$ e $90^{\circ}$, porém, as mudanças nas direções ao redor dessas fileiras são menos abruptas se comparadas aos 
dois resultados obtidos utilizando $r=5 \mathrm{~mm}$ (ver Figs. 6.3a e 6.3b). Considerando $r=30 \mathrm{~mm}$ (ver Figs. 6.3e e 6.3f) obtêm-se um resultado onde as alterações nas direções das fibras ocorrem de forma suave se comparadas aos resultados anteriores (ver Figs. 6.3a a 6.3d).

A Fig. 6.4 demonstra a convergência da função objetivo durante o processo de otimização. Observa-se que, apesar de um $r$ maior garantir uma melhor continuidade nas fibras, 0 aumento deste parâmetro também implica em um aumento no valor da função objetivo e, em alguns casos, em uma pior estabilidade na convergência. Como pode ser visto na Tabela 6.2, o menor valor de função objetivo é obtido quando $r=5 \mathrm{~mm}$ e o chute inicial utilizado é $\theta_{0}=0^{\circ}$. Para o mesmo valor de parâmetro $r$ e chute inicial $\theta_{0}=45^{\circ}$, o valor obtido é $0,07 \%$ maior. Já para quando o parâmetro $r=15 \mathrm{~mm}$ considerando o a distribuição inicial de $\theta_{0}=0^{\circ}$, o valor da função objetivo é $1,7 \%$ que o melhor resultado obtido, enquanto que para $r=15 \mathrm{~mm}$ e $\theta_{0}=45^{\circ}$, o resultado é $1,8 \%$ maior. Os resultados para quando $r=30 \mathrm{~mm}$ apresentam os maiores valores de função objetivo, sendo o resultado para distribuição inicial de $\theta_{0}=45^{\circ} 4,6 \%$ maior e para a distribuição inicial de $\theta_{0}=0^{\circ}, 5,0 \%$ maior.

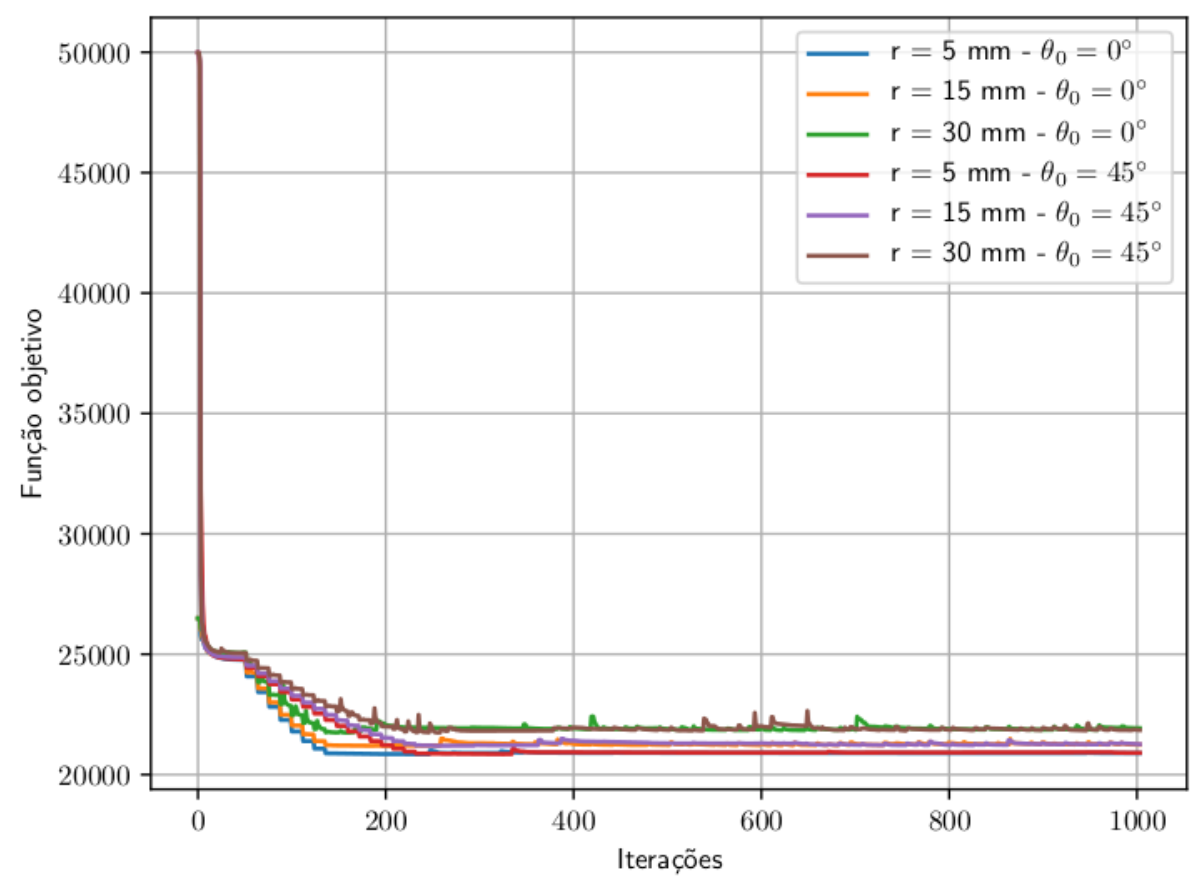

Figura 6.4 - Convergência da função objetivo para a otimização da viga engastada.

Como pode ser observado nos resultados apresentados na Fig. 6.3, as direções das fibras são similares as direções de maior tensão principal deste mesmo domínio considerando um material isotrópico. Gea e Luo (2004) propõe um método para prever as direções otimizadas das orientações de fibras baseadas nas direções principais de tensão e deformação. O resultado apresentado a seguir, é obtido considerando como distribuição inicial as direções de maior tensão principal de um material isotrópico, ocupando o mesmo 
Tabela 6.2 - Valor final da função objetivo para viga engastada.

\begin{tabular}{ccc}
\hline$r[\mathrm{~mm}]$ & $\theta_{0}\left[^{\circ}\right]$ & Função objetivo $[\mathrm{Nmm}]$ \\
\hline 5 & 0 & 20,891 \\
5 & 45 & 20,906 \\
15 & 0 & 21,244 \\
15 & 45 & 21,263 \\
30 & 45 & 21,851 \\
30 & 0 & 21,929 \\
\hline
\end{tabular}

domínio apresentado na Fig. 6.2a. Essa distribuição inicial é apresentada na Fig. 6.5a e o resultado obtido pode ser observado na Fig. 6.5b.

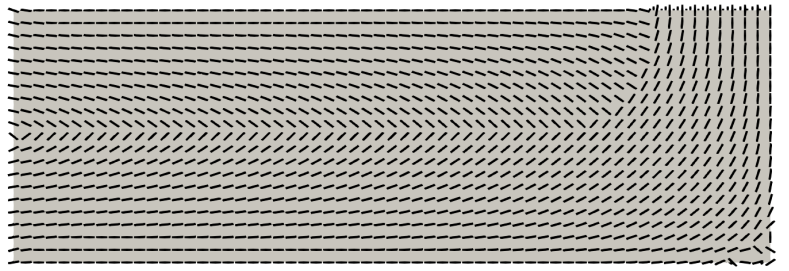

(a) Direções principais considerando um material isotrópico.

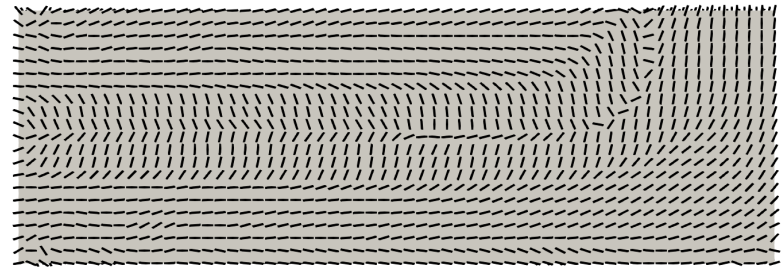

(b) Resultado para $r=15 \mathrm{~mm}$

Figura 6.5 - Otimização para distribuição inicial nas direções de maior tensão principal para um material isotrópico.

A Fig. 6.6 demonstra a convergência para três diferentes distribuições iniciais e $r=$ $15 \mathrm{~mm}$. Para este exemplo, a distribuição inicial de $45^{\circ}$ inicia com o valor mais distante do otimizado, enquanto a distribuição nas direções principais é a que inicia mais próxima ao valor otimizado. Porém, os resultados otimizados obtidos são muito próximos como demonstra a Tabela 6.3, onde verifica-se que o valor de função objetivo para $\theta_{0}=45^{\circ}$ é apenas $0,09 \%$ maior que o menor valor obtido (quando $\theta_{0}=0^{\circ}$ ) e que para o chute nas direções de maior tensão principal é apenas $0,19 \%$ maior.

Tabela 6.3 - Comparação entre distribuições iniciais.

\begin{tabular}{ccc}
\hline$r[\mathrm{~mm}]$ & $\theta_{0}\left[^{\circ}\right]$ & Função objetivo $[N \mathrm{~mm}]$ \\
\hline 15 & 0 & 21,244 \\
15 & 45 & 21,263 \\
15 & Direção de maior tensão principal & 21,285 \\
\hline
\end{tabular}

\subsubsection{Viga simplesmente apoiada}

No segundo exemplo é realizada a otimização na direção das fibras em uma viga simplesmente apoiada onde uma força de $F=200 \mathrm{kN} / \mathrm{m}^{2}$ é aplicada. As condições de 


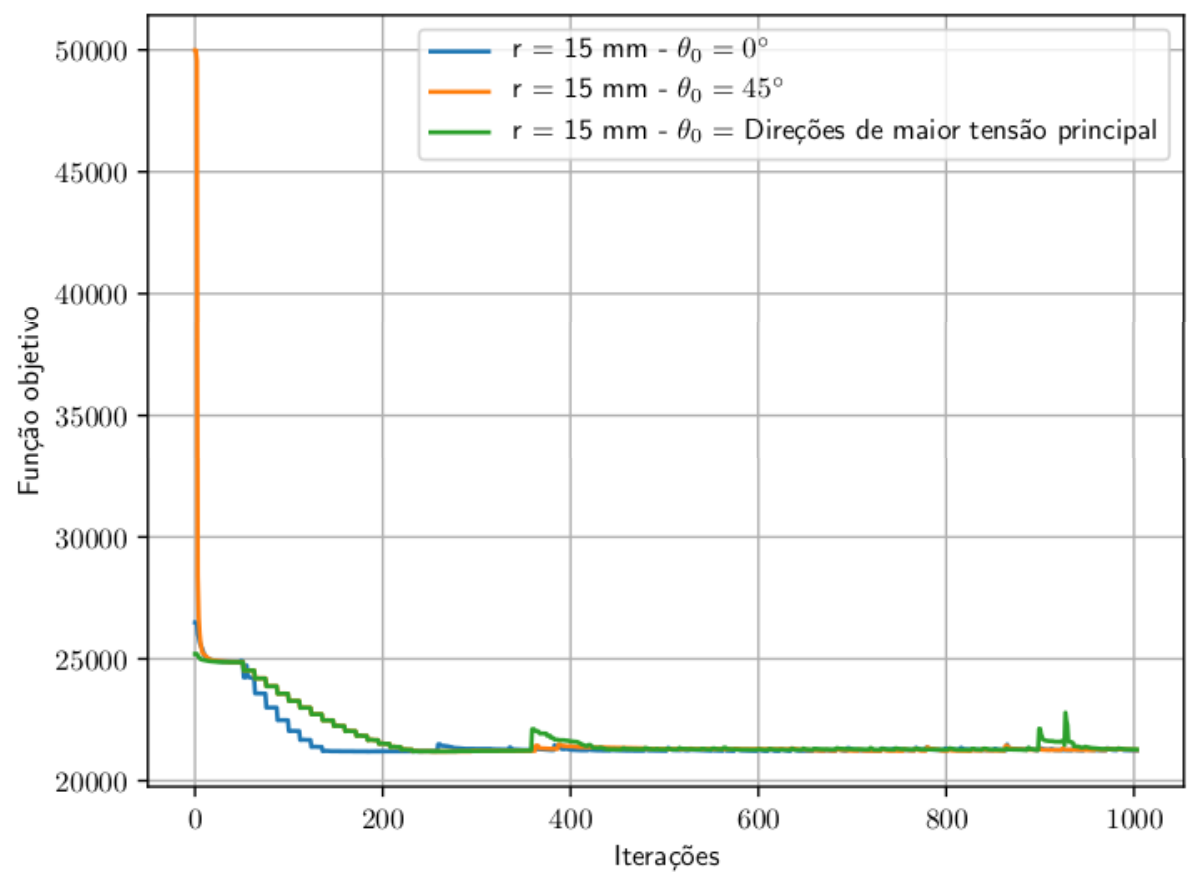

Figura 6.6 - Convergências das funções objetivo considerando $r=15 \mathrm{~mm}$

contorno consideradas são apresentadas na Fig. 6.7a enquanto que, na Fig. 6.7b há a representação de um resultado tridimensional com fibras longas. As propriedades do material, ângulos candidatos, número de iterações e esquemas de continuação são os mesmo utilizados na Seção 6.1.1.

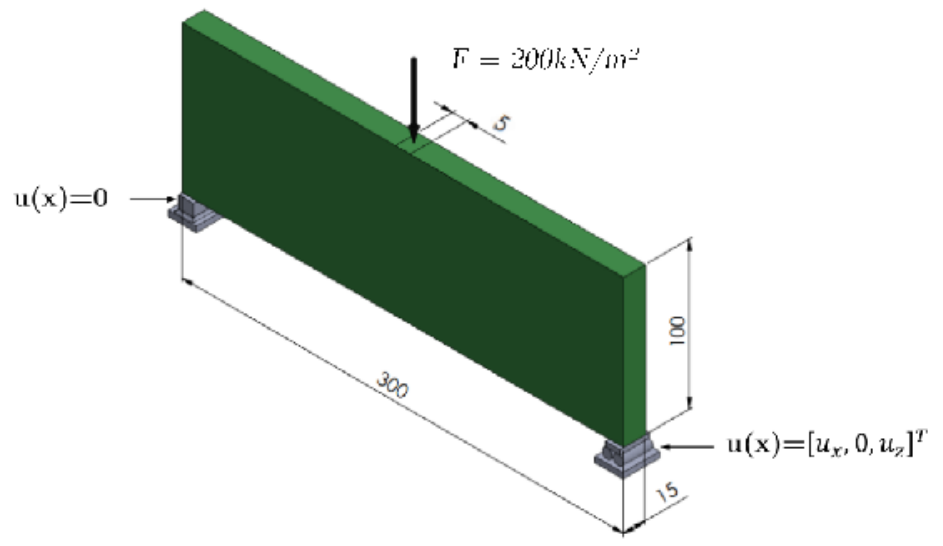

(a) Domínio da viga simplesmente apoiada.

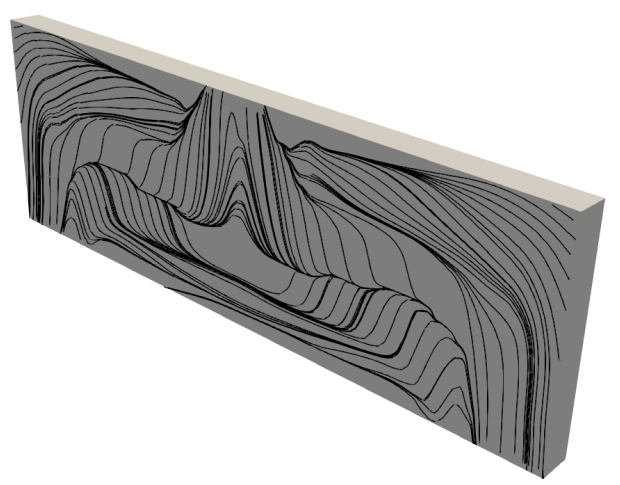

(b) Resultado tridimensional.

Figura 6.7 - Viga simplesmente apoiada.

Por meio dos resultados apresentados nas Figs. 6.8a a 6.8c, observa-se novamente a tendência do alinhamento das fibras com a força aplicada e com os apoio da viga. Esse alinhamento faz com que o deslocamento seja o menor possível, uma vez que o valor do 
modulo de Young é maior na direção $E_{1}$ (direção das fibras), maximizando assim, a rigidez da estrutura.

As Figs. $6.8 \mathrm{a}$ a $6.8 \mathrm{c}$ representam os resultados obtidos por meio do modelo OFDN-m e analisando-as, pode-se novamente avaliar o efeito da mudança do parâmetro $r$ do filtro de Helmholtz no resultado otimizado. Com $r=5 \mathrm{~mm}$ (ver Fig. 6.8a), observa-se fibras posicionadas verticalmente nos locais onde a viga está apoiada (extremidades direita e esquerda da parte inferior). Partindo dessas extremidades, forma-se um caminho similar ao formato de uma asa, onde as fibras tem ângulos próximos aos candidatos com menor e maior valor, que se estende até o local de aplicação da força. Algumas mudanças bruscas nas direções das fibras ocorrem na região próxima a aplicação da força e ao longo do padrão similar a uma asa. Quando $r=15 \mathrm{~mm}$ (ver Fig. 6.8b), um padrão similar ao apresentado no resultado anterior (ver Fig. 6.8a) é observado, porém, as fibras com ângulos próximos ou iguais a $-78.75^{\circ}$ e $90^{\circ}$ ocupam uma região maior do domínio. Mudanças abruptas na direção das fibras são observadas, novamente, na região próxima a aplicação da força e em algumas partes do caminho similar ao formato de asa. O resultado obtido para $r=30 \mathrm{~mm}$ é apresentado na Fig. 6.8c, onde pode-se observar que a maior parte do domínio possui fibras com ângulos iguais aos candidatos de valor mínimo e máximo. Neste resultado, as mudanças de direção abruptas próximas a aplicação da força e no padrão em forma de asa são mitigadas.

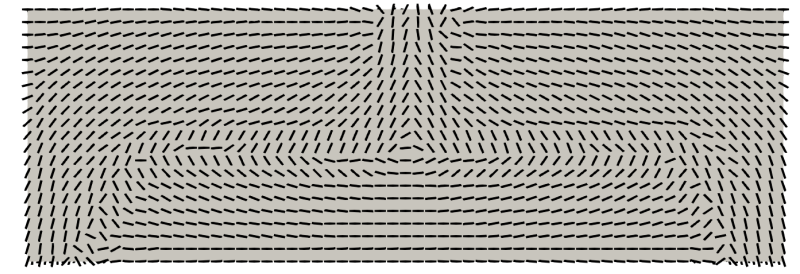

(a) Resultado do modelo OFDN-m com $r=5 \mathrm{~mm}$

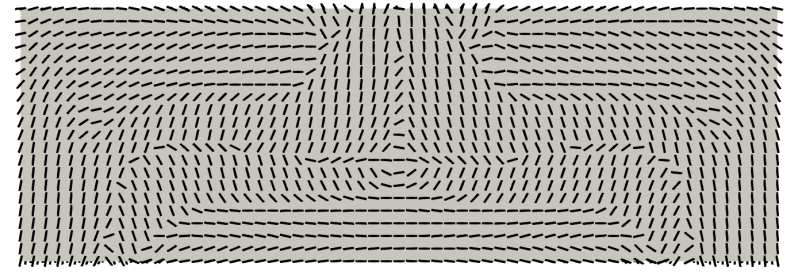

(b) Resultado do modelo OFDN-m com $r=15 \mathrm{~mm}$

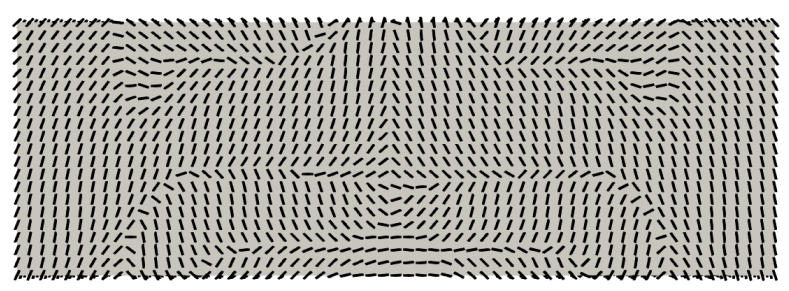

(c) Resultado do modelo OFDN-m com $r=30 \mathrm{~mm}$

Figura 6.8 - Orientação otimizada das fibras para uma viga apoiada sujeita a uma carga de $F=200 \mathrm{kN} / \mathrm{m}^{2}$ obtida com o modelo OFDN-m

As convergências da função objetivo para a viga simplesmente apoiada são apresentadas na Fig. 6.9. A estabilidade na convergência foi alcançada apenas para o exemplo onde $r=5 \mathrm{~mm}$, onde novamente obteve-se o menor valor para função objetivo (ver Tabela 6.4). 0 valor da função objetivo na última iteração para $r=15 \mathrm{~mm}$ (ver Fig. 6.8b) é 10,0\% maior que 
o valor obtido para $r=5 \mathrm{~mm}$ (Fig. 6.8a). Para o caso onde $r=30 \mathrm{~mm}$ (ver Fig. 6.8c), o valor da função objetivo na última iteração é $27,5 \%$ maior.

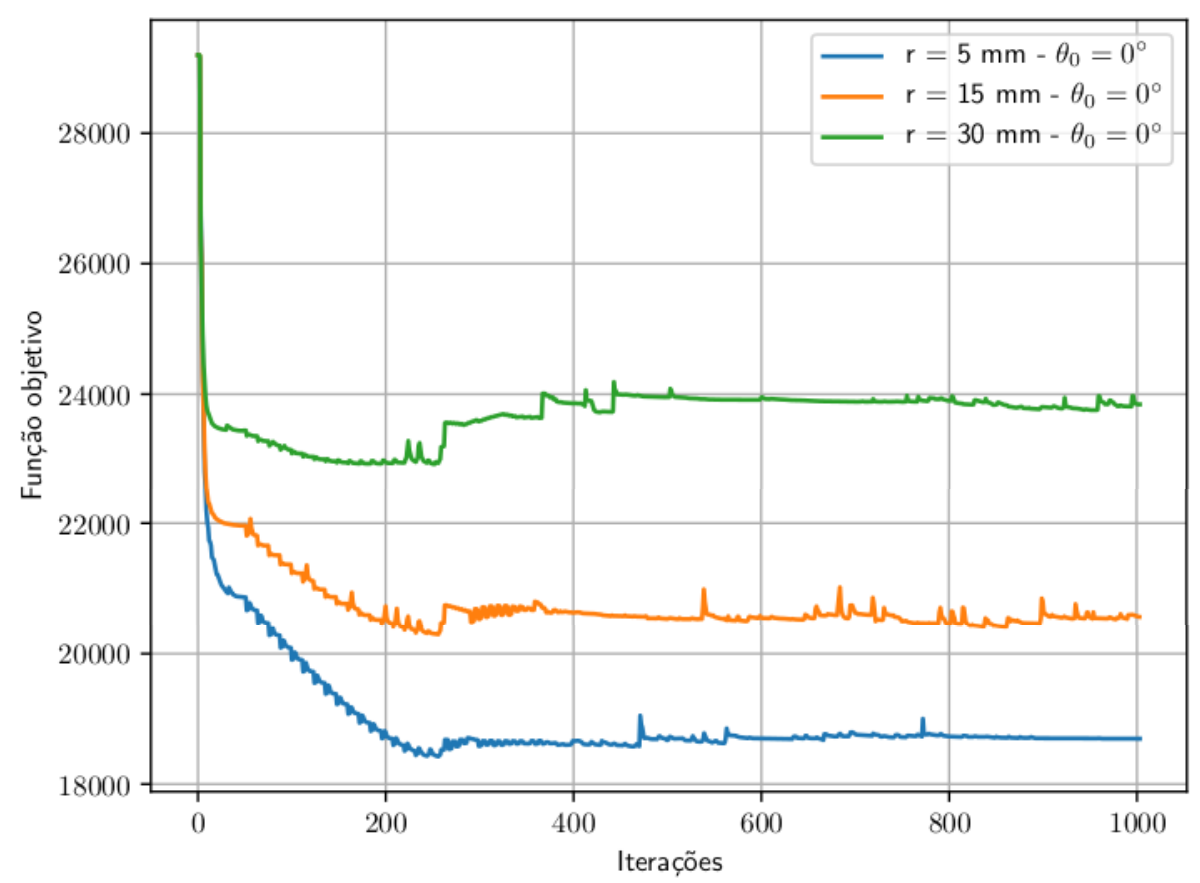

Figura 6.9 - Convergência da função objetivo para a otimização da viga simplesmente apoiada.

Tabela 6.4 - Valor final da função objetivo para viga bi-apoiada.

\begin{tabular}{ccc}
\hline$r[\mathrm{~mm}]$ & $\theta_{0}\left[^{\circ}\right]$ & Função objetivo $[\mathrm{N} \mathrm{mm}]$ \\
\hline 5 & 0 & 18,696 \\
15 & 0 & 20,573 \\
30 & 0 & 23,842 \\
\hline
\end{tabular}

\subsubsection{Placa com força fora do plano}

O terceiro e último exemplo consiste em uma placa fixada nas quatro extremidades com uma força de $F=300 \mathrm{kN} / \mathrm{m}^{2}$ aplicada fora do plano. As propriedades do material, esquemas de continuação, número de iterações e ângulos candidatos são os mesmos utilizados na Seção 6.1.1. Na Fig. 6.10 são apresentados o domínio, condições de contorno e um resultado tridimensional com fibras longas.

A Fig. 6.11 demonstra os resultados onde os valores utilizados para $r$ são novamente $5 \mathrm{~mm}, 15 \mathrm{~mm}$ e $30 \mathrm{~mm}$. 


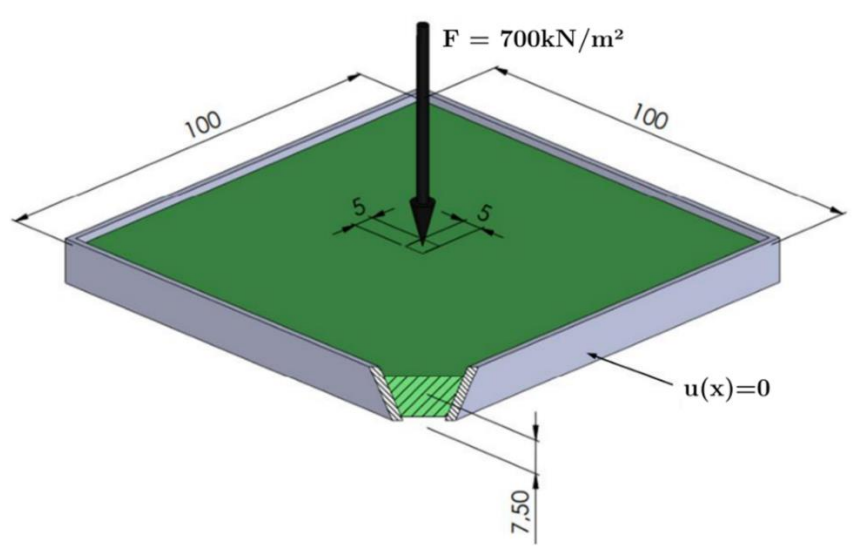

(a) Domínio da placa fixa.

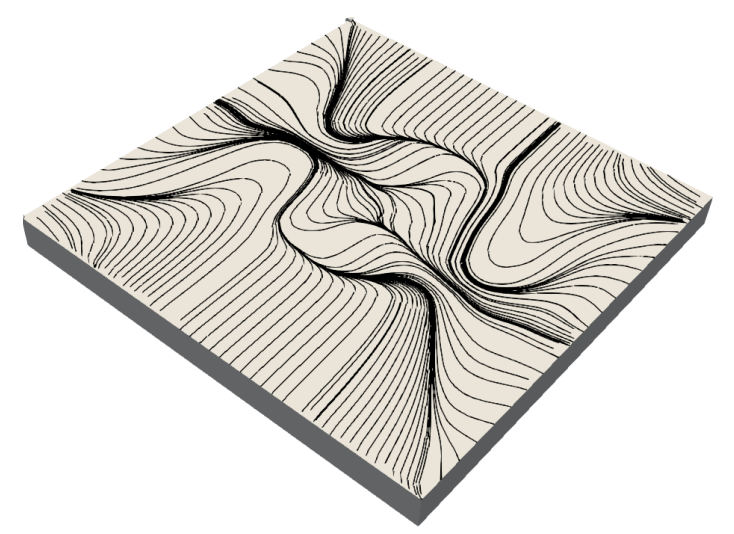

(b) Representação de um resultado tridimensional.

Figura 6.10 - Placa fixa com força fora do plano.

Para este exemplo, não existe a possibilidade das fibras ficarem alinhas com a direção de aplicação da força, já que a carga é aplicada fora do plano e as orientações são customizadas no plano. Porém, as fibras formam novamente a orientação que proporciona o menor deslocamento, tornando a estrutura mais rígida.

Observando os resultados, percebe-se na Fig. 6.11a a presença de uma circunferência central com descontinuidades em seu interior. Porém, com o aumento do parâmetro $r$, as descontinuidades diminuem, como pode ser visto em Figs. $6.11 \mathrm{~b}$ e $6.11 \mathrm{c}$. O resultado para $r=15 \mathrm{~mm}$ (ver Fig. 6.11b) apresenta algumas mudanças abruptas na direção das fibras, principalmente ao redor do padrão, agora elíptico, presente no interior do domínio. Quando $r=30 \mathrm{~mm}$ percebe-se que as mudanças abruptas nas direções das fibras são mitigadas.

A convergência da função objetivo é apresentada na Fig. 6.12. A convergência não alcançou a estabilidade para os exemplos onde $r=15 \mathrm{~mm}$ e $r=30 \mathrm{~mm}$ e, assim como nos exemplos anteriores, o menor valor da função objetivo é obtido a partir do menor valor de $r$. Em comparação com o valor da última iteração considerada quando $r=5 \mathrm{~mm}$ (Fig. 6.11a), o resultado para $r=15 \mathrm{~mm}$ (Fig. 6.11b) é aproximadamente 1,70\% maior. Quando comparados os valores obtidos para $r=30 \mathrm{~mm}$ (Fig. 6.11c) com a última iteração do resultado com $r=5 \mathrm{~mm}$ (Fig. 6.11a), tem-se um valor 4,97\% maior.

Tabela 6.5 - Valor final da função objetivo para Placa com força fora do plano.

\begin{tabular}{ccc}
\hline$r[\mathrm{~mm}]$ & $\theta_{0}\left[^{\circ}\right]$ & Função objetivo $[\mathrm{N} \mathrm{mm}]$ \\
\hline 5 & 0 & 20,891 \\
15 & 0 & 21,244 \\
30 & 0 & 21,929 \\
\hline
\end{tabular}




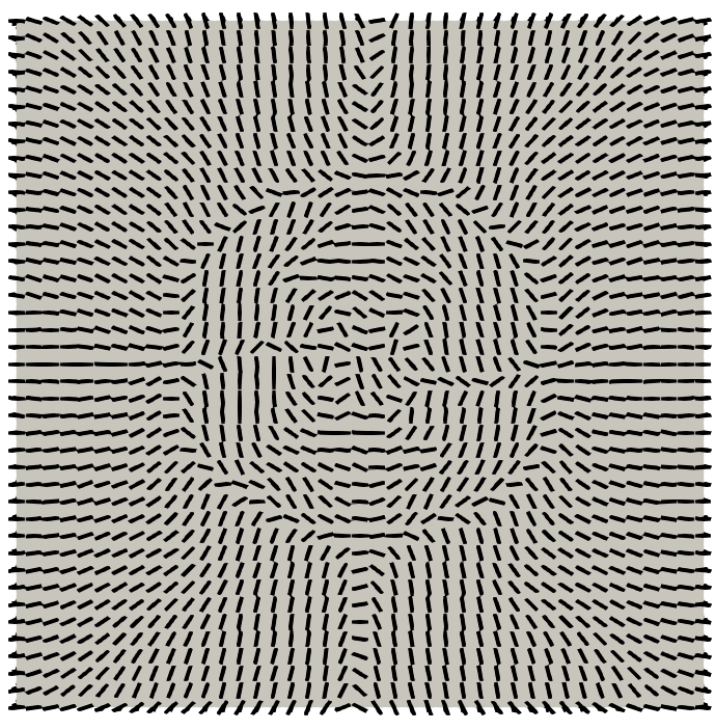

(a) Resultado do modelo OFDN-m com $r=5 \mathrm{~mm}$

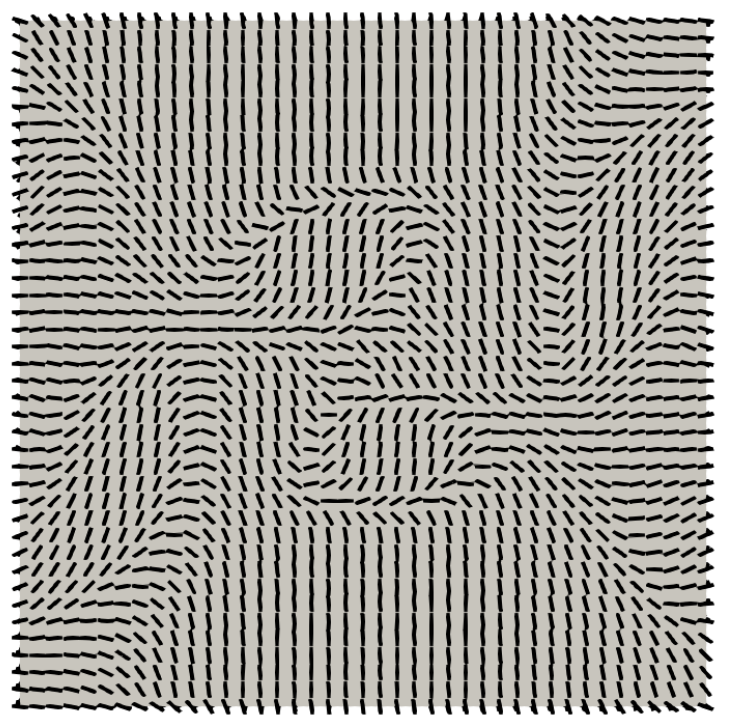

(b) Resultado do modelo OFDN-m com $r=15 \mathrm{~mm}$

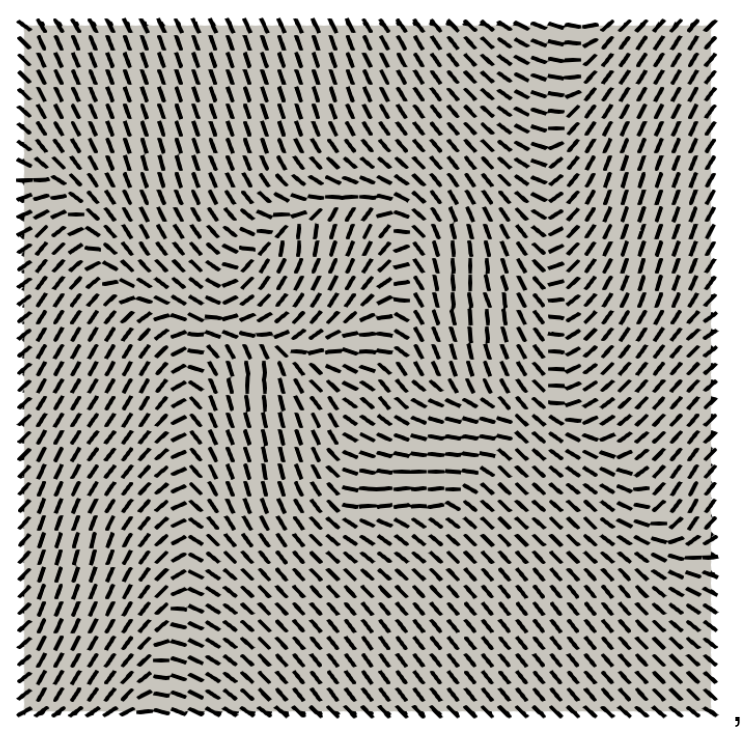

(c) Resultado do modelo OFDN-m com $r=30 \mathrm{~mm}$

Figura 6.11 - Orientação otimizada das fibras para uma placa sujeita a uma carga de $F=300 \mathrm{kN} / \mathrm{m}^{2}$ fora do plano.

\subsection{Resultados de OT aplicada simultaneamente a distribuição de material e orientação de fibras}

Os resultados onde a orientação das fibras é determinada simultaneamente à distribuição de material no domínio são obtidos utilizando-se o modelo MIAFIB. Três exemplos numéricos são apresentados, sendo o primeiro uma viga em balanço com carga aplicada na extremidade (Seção 6.2.1), o segundo, um exemplo de viga "MBB (Messerschmitt-Bolkow-Blohm)" (Seção 6.2.2) e o último, uma estrutura "L" (Seção 6.2.3). A fração de volume para todos os exemplos é $V=0.6$ e o material considerado é o epoxy reforçado com fibra de vidro (ver (KAW, 2005)). As propriedades do material e parâmetros para os modelos MIAFIB e SIMP 


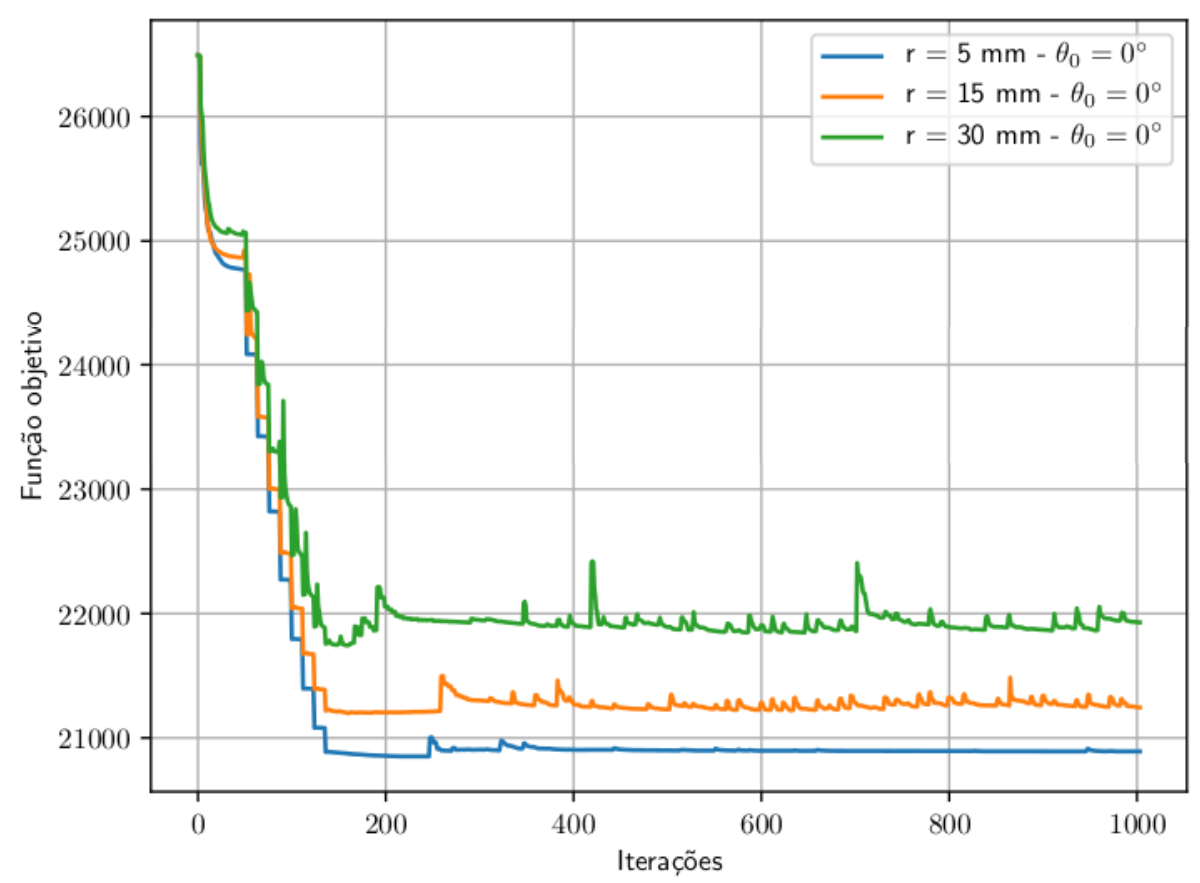

Figura 6.12 - Convergência da função objetivo para a otimização da placa com força fora do plano.

são apresentados na Tabela 6.6

\begin{tabular}{cccc|cccc|cc}
\hline \multicolumn{3}{c|}{ Propriedades do material } & \multicolumn{4}{c|}{ MIAFIB } & \multicolumn{3}{c}{ SIMP } \\
\hline$E_{1}[G P a]$ & $E_{2}[G P a]$ & $G_{12}[G P a]$ & $v_{12}$ & $\hat{\theta}_{c}^{\min }$ & $\hat{\theta}_{c}^{\text {max }}$ & $p_{f}^{\text {ini }}$ & $p_{f}^{\text {max }}$ & $p_{\text {ini }}$ & $p_{\text {max }}$ \\
\hline 38,6 & 8,27 & 4,14 & 0,26 & $-89 / 180$ & $90 / 180$ & 2 & 17 & 1 & 6 \\
\hline
\end{tabular}

Tabela 6.6 - Propriedades do material e parâmetros dos modelos de material Seção 6.2.

A malha de MEF utilizada nestas simulações é regular e composta por elementos triangulares com interpolação linear de dimensões $2,5 \mathrm{~mm} \times 2,5 \mathrm{~mm}$. Os mesmos elementos são utilizados para a determinação dos deslocamentos, pseudo-orientação e pseudo-densidades (ver Fig. 6.13)

\subsubsection{Viga em balanço}

O primeiro exemplo solucionado é apresentado na Fig. 6.14. A região hachurada representa a condição de contorno de Dirichlet, as linhas verdes representam a distribuição inicial de fibras $\left(\theta_{0}=0^{\circ}\right)$ e a região cinza representa a distribuição inicial de material $(\bar{\rho}=0,6)$. Para este exemplo, o valor dos parâmetros do filtro de Helmholtz é 10 para as duas variáveis de projeto. A função objetivo é avaliada 1000 vezes, porém, a restrição de continuidade (ver 

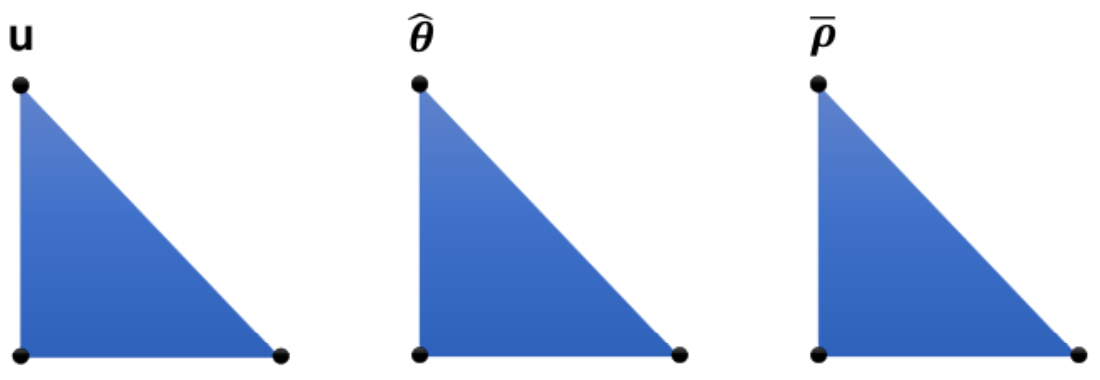

Figura 6.13 - Elementos triangulares com interpolação linear.

Seção 3.6.3 e Eq. (4.3)) é levada em conta a partir da iteração $i_{\text {Borrvall }}=800$, onde não há grande presença de cinza na distribuição de material.

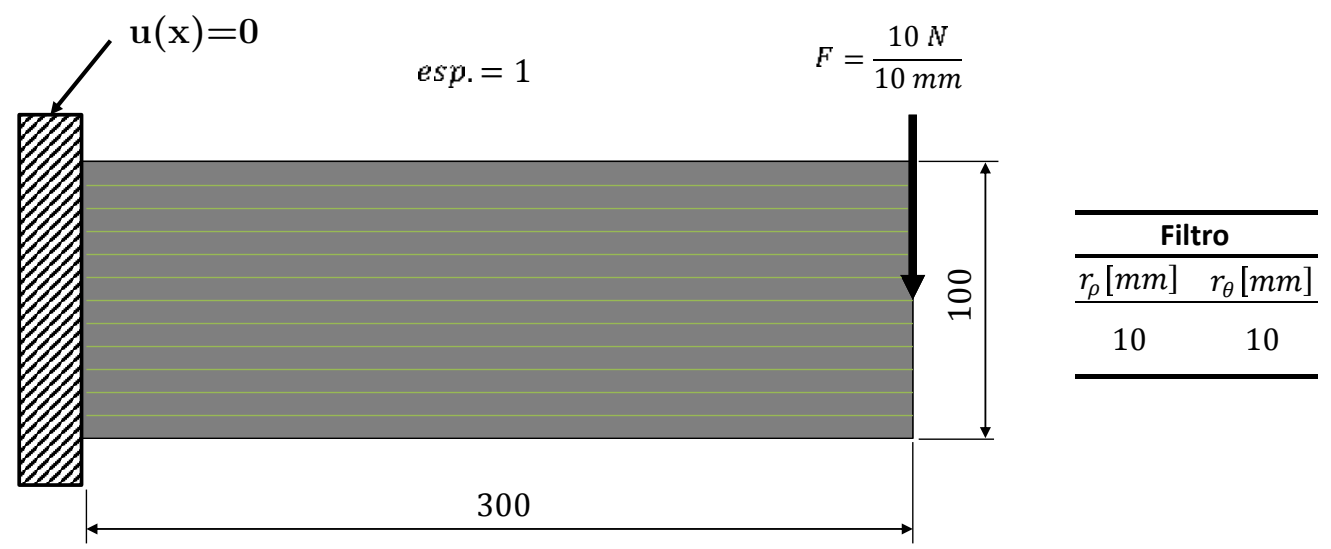

Figura 6.14 - Domínio da viga em balanço.

Como apresentado na Tabela 6.6, o penalizador $p_{f}$ do modelo MIAFIB varia entre $p_{f}^{f}=2$ e $p_{f}^{i}=17$ e a continuação a ele aplicada é baseada na Eq. (6.1).

$$
s_{p}^{M I A F I B}=\left\lfloor\frac{i_{\max }^{M I A F I B}}{p_{f}^{f}-p_{f}^{i}+1}\right\rfloor
$$

onde $s_{p}^{M I A F I B}$ representa o número de iterações em que cada $p_{f}$ é utilizado até que as iterações atinjam um valor previamente definido igual a $i_{\max }^{M I A F I B}$. Neste exemplo, $i_{\max }^{M I A F I B}=800$, portanto, o valor de $p_{f}$ que inicialmente é igual a 2, aumenta em uma unidade a cada 50 iterações até atingir o valor de 17.

Para o modelo SIMP, é recomendado que se inicie com $p^{i n i}=1$ e que o valor de $p$ aumente lentamente até que atinja um valor previamente definido igual $p^{\max }$ (BENDS $\varnothing \mathrm{E}$; SIGMUND, 2003), portanto, a continuação para o penalizador $p$ é definida de acordo com a 
Eq. (6.2)

$$
\begin{array}{r}
p_{i+1}=p_{i}+\Delta p \\
\Delta p=\frac{p^{\max }-p^{i n i}}{i_{\max }^{S I M P}-1}
\end{array}
$$

onde $\Delta p$ é o incremento para o penalizador $p$ e $i_{\max }^{S I M P}$, o qual é previamente definido, é o número de iterações necessárias para que o valor de $p$ varie entre o valor inicial e o valor máximo. Para este exemplo, $i_{\max }^{\text {SIMP }}=800$.

A Fig. 6.15 representa o resultado obtido onde uma fibra reta de pequeno comprimento é representada em cada nó a malha de elementos finitos, na iteração 800 , onde a restrição de continuidade ainda não foi considerada. A legenda com escala de cinza a direita, representa os valores do campo de pseudo-densidades $(\bar{\rho})$.

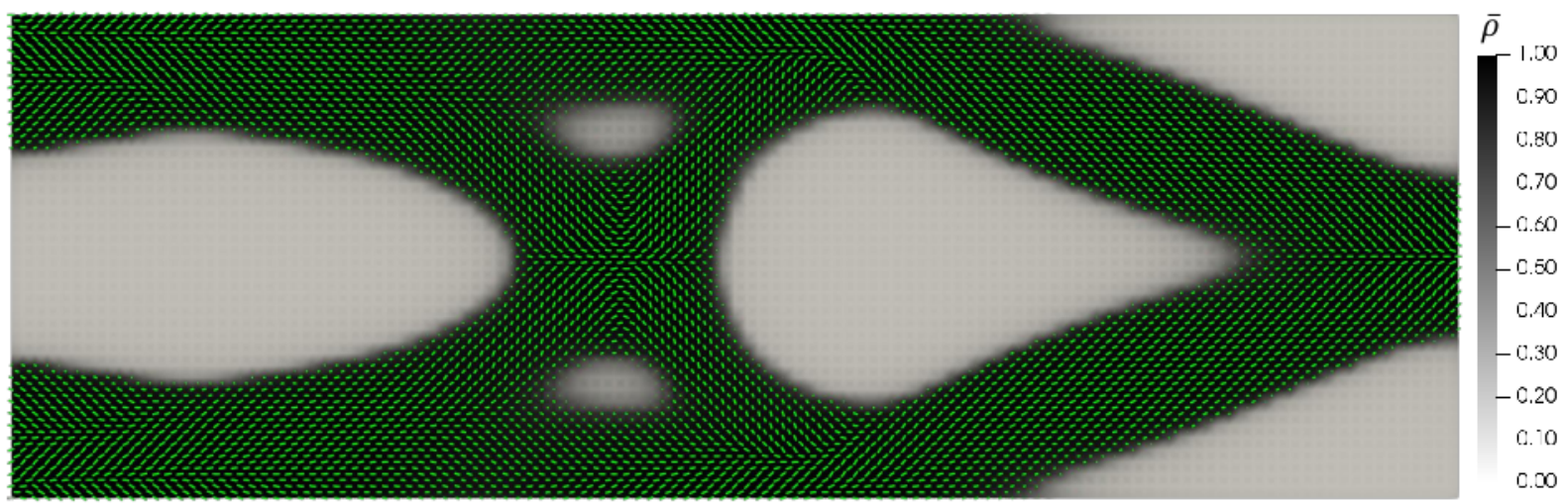

Figura 6.15 - Orientação de fibras e distribuição de material otimizadas para a viga em balanço.

O resultado apresentado na Fig. 6.15 deve ser interpretado considerando fibras longas, e para isso, um pós-processamento é realizado no software de visualização de dados ParaView. A interpretação do resultado apresentado na Fig. 6.15 é realizada utilizando-se um filtro denominado Stream Tracer, o qual gera linhas de corrente a partir de campos vetoriais (para maiores detalhes sobre o filtro Stream Tracer, ver (AYACHIT, 2015)). Uma possível interpretação do resultado para o exemplo da viga em balanço (Fig. 6.15) é apresentada na Fig. 6.16

Pode-se observar na Fig. 6.16 que as mudanças nas direções das fibras, são consideravelmente suaves, no entanto, há acumulo de fibras em certas regiões do domínio. Para mitigar o problema de acumulo de fibras, a partir da iteração 800 , considera-se a restrição de continuidade (Eq. (3.29)) com as condições de contorno para o modelo de Borrvall apresentadas na Fig. 6.17. O valor de $\beta$ é determinado em função da área do domínio, sendo $\beta^{2}=\frac{\pi}{180 \text { area } a_{\text {dom }}}$. A velocidade entra do lado esquerdo do domínio com um perfil linear e valor $\overline{\mathbf{v}}_{l}=\left[\begin{array}{ll}0.01 & 0\end{array}\right]^{T} \mathrm{~mm} / \mathrm{s}$ e do lado direito é definida a pressão $\bar{p}=0$. Nas fronteiras 


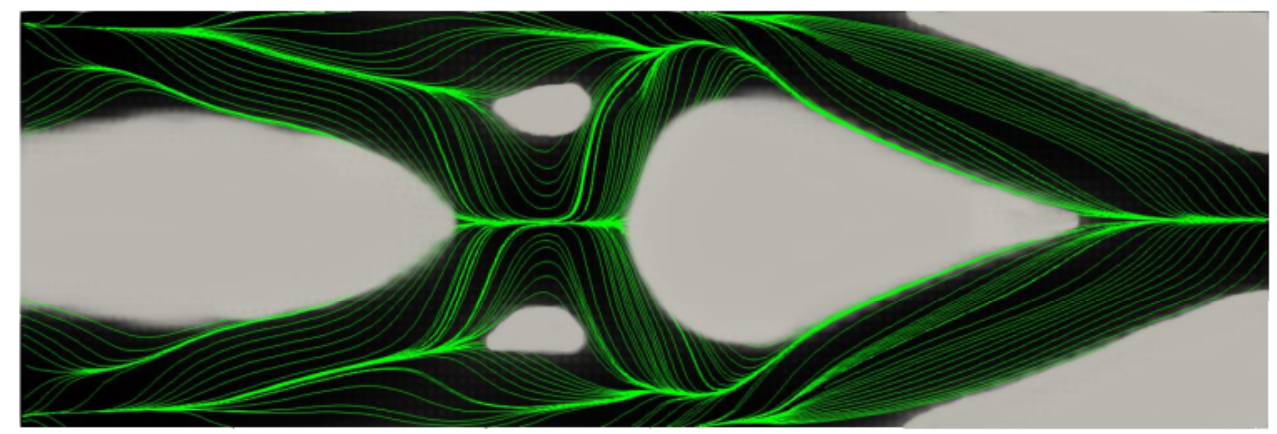

Figura 6.16 - Interpretação do resultado para a viga em balanço.

superior e inferior do domínio são impostas as condições de não escorregamento $\left(\overline{\mathbf{v}}_{l}=\mathbf{0}\right)$. Os parâmetros para o modelo de Borrvall considerados são $\bar{\alpha}=50, \underline{\alpha}=0, \mu_{l}=1 \mathrm{~N} / \mathrm{mm} \cdot \mathrm{s}$, $q=0.1$ e $\rho_{l}=\bar{\rho}$. O elemento finito para velocidade $\overline{\mathbf{v}}_{l}$ é o triangular com interpolação quadrática e para pressão $\bar{p}$ utiliza-se o elemento triangular com interpolação linear (ver Fig. 6.17).

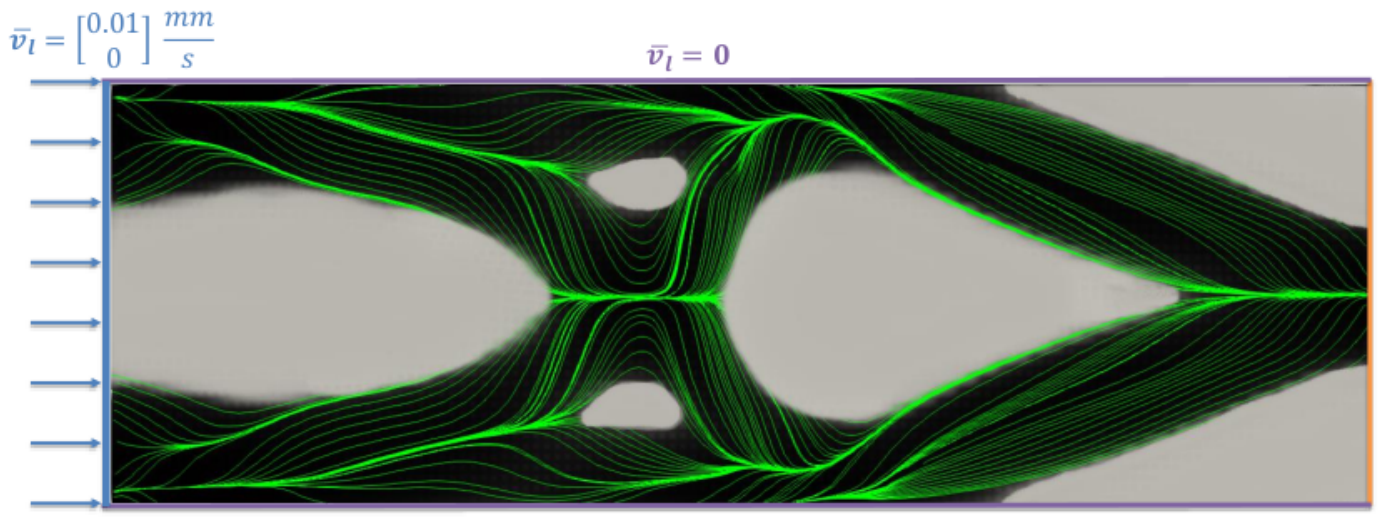

$\bar{v}_{l}=0$

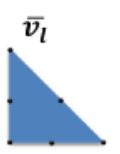

$2.5 \times 2.5$

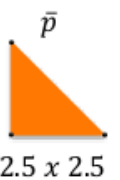

$2.5 \times 2.5$

Figura 6.17 - Condições de contorno para o modelo de Borrvall no problema da viga em balanço.

As Figs. 6.18a e 6.18b demonstram o resultado da otimização para a viga em balanço considerando a restrição de continuidade e o campo de velocidades obtido no modelo de Borrvall na última iteração respectivamente. Uma comparação entre as velocidades e as fibras é apresentada na Fig. 6.18c, onde pode-se observar que para a maior parte do domínio, os ângulos das fibras são muito próximos aos vetores de velocidade, com exceção a duas regiões, próximas a fronteiras no centro do domínio. Em função da similaridade do campo de velocidades com ângulo das fibras, opta-se por realizar o pós-processamento nas velocidades, por estas apresentarem uma melhor continuidade. Uma possível interpretação para o resultado obtido utilizando a restrição de continuidade é apresentada na Fig. 6.18d.

Percebe-se por meio da Fig. 6.18 d que o resultado obtido utilizando a restrição de continuidade apresenta mudanças suaves na direção das fibras. Comparando as Figs. 6.16 
e 6.18d, pode-se concluir que após a aplicação da restrição de continuidade, o problema de acumulo de fibras foi mitigado.

\subsubsection{Viga MBB}

O segundo exemplo é o de uma viga MBB e é apresentado na Fig. 6.19. O domínio completo da viga é apresentado na Fig. 6.19a onde novamente as linhas verdes representam a distribuição inicial para as fibras $\left(\theta_{0}=0^{\circ}\right)$ e o cinza representa a distribuição inicial da pseudo-densidade $(\bar{\rho}=0,6)$. Para a solução deste exemplo, utiliza-se metade do domínio com as condições de contorno apresentadas na Fig. 6.19b. Os parâmetros do filtro de Helmholtz são $r_{\bar{\rho}}=20 \mathrm{~mm}$ e $r_{\theta}=30 \mathrm{~mm}$. Assim como na Seção 6.2.1, a função objetivo é avaliada 1000 vezes e a restrição de continuidade passa a ser considerada na iteração 800 .

O resultado obtido após 800 iterações, sem considerar a restrição de continuidade, é apresentado na Fig. 6.20. A Fig. 6.20a representa o resultado obtido, com fibras de pequeno comprimento em cada nó da malha de elementos finitos e a Fig. 6.20b é uma possível interpretação do resultado obtido.

Por meio da representação de fibras longas apresentada na Fig. 6.20b pode-se observar mudanças abruptas na direção das fibras em uma região do domínio próxima a aplicação da força. Além disso, nota-se um evidente acumulo de fibras em determinadas áreas.

A restrição de continuidade é utilizada a partir da iteração 800 para tratar as questões de mudança de direções e acumulo de fibras com as condições de contorno e elementos apresentados na Fig. 6.21 e os mesmos parâmetros utilizados na Seção 6.2.1.

O resultado obtido considerando a restrição de continuidade, com uma fibra pequena em cada nó da malha de elementos finitos, é apresentado na Fig. 6.22a. Já o campo de velocidades obtido no modelo de Borrvall é apresentado na Fig. 6.22b e a comparação entre essas velocidades e o angulo das fibras é apresentada da Fig. 6.22c. Observa-se na Fig. 6.22c que as fibras apresentam a mesma direção que o campo de velocidades em praticamente todo o domínio, com exceção das fronteiras onde são impostas as condições de não-escorregamento. Isto ocorre em razão da restrição de continuidade sempre estar sendo respeitada nessas fronteiras, independentemente do valor de $\theta$ (ver Eq. (3.29)). Uma possível interpretação para o resultado da viga MBB é apresentada na Fig. 6.22d, onde novamente, em função da similaridade entre fibras e velocidades e pela melhor continuidade, o pós-processamento foi realizado no campo de velocidades. É possível verificar que o caminho formado pelas fibras possui mudanças de direções mais suaves se comparadas com as apresentadas na Fig. 6.20b e que o problema de acumulo das fibras pôde ser tratado. 


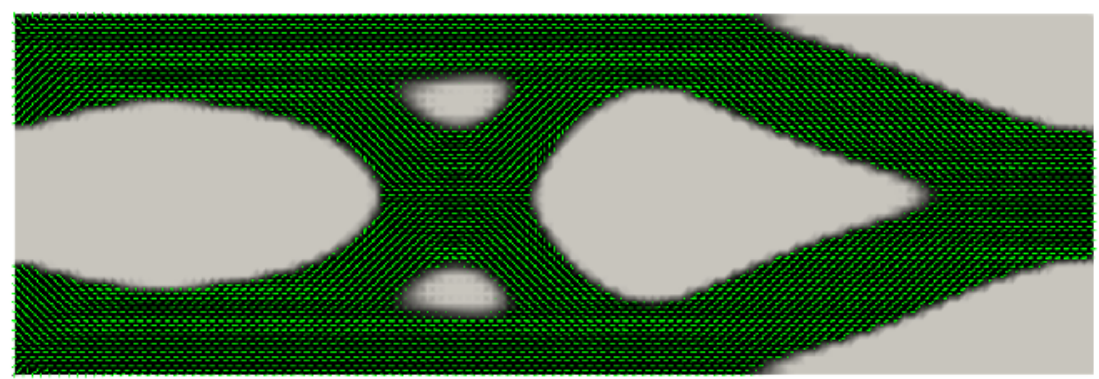

(a) Resultado otimizado com restrição de continuidade.

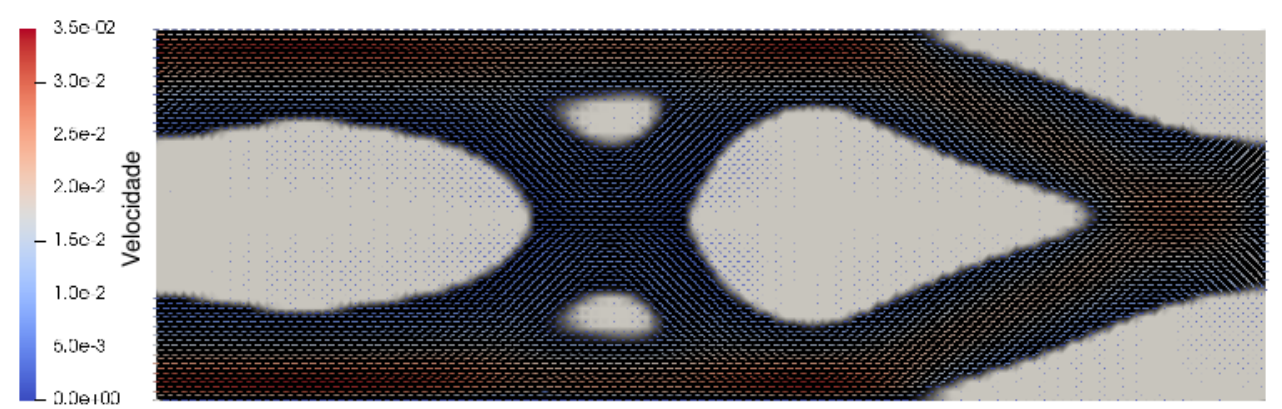

(b) Campo de velocidades.

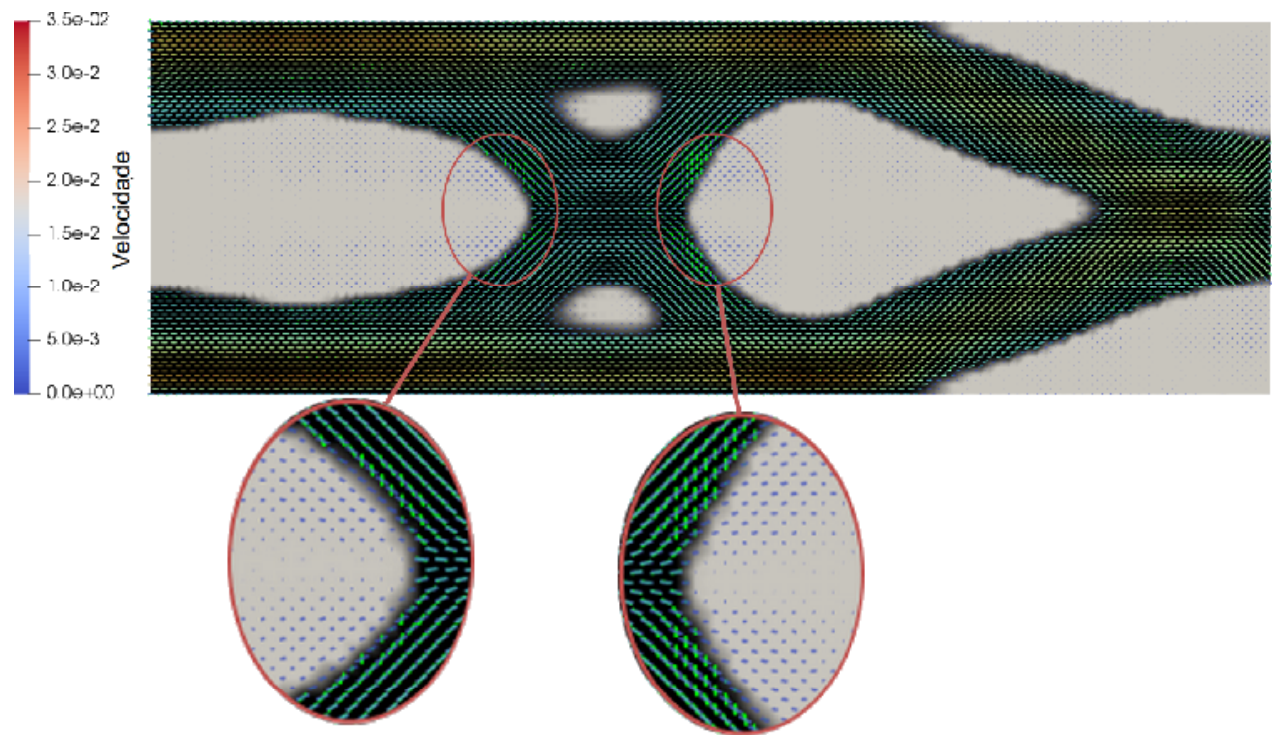

(c) Comparação entre velocidades e fibras.

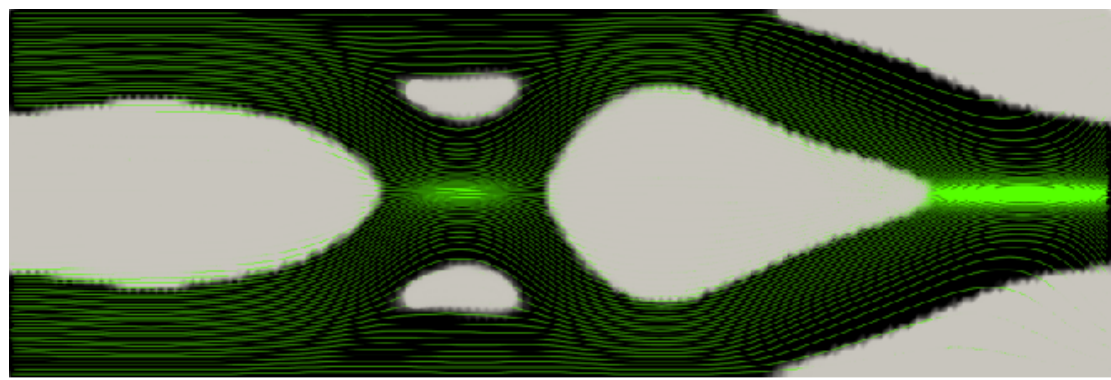

(d) Interpretação do resultado.

Figura 6.18 - Resultado para a viga em balanço com restrição de continuidade. 


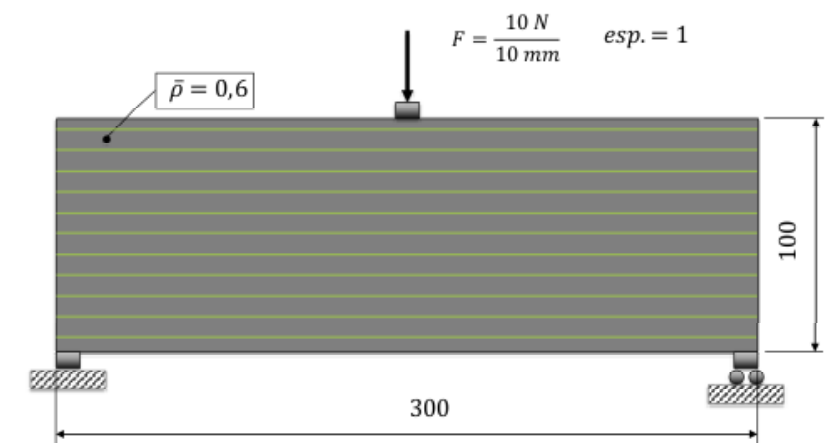

(a) Domínio completo para a viga MBB.

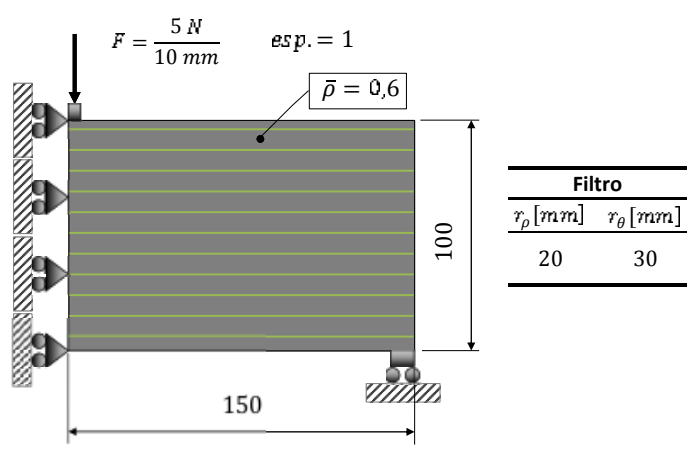

(b) Meio domínio para a viga MBB.

Figura 6.19 - Domínio da viga MBB
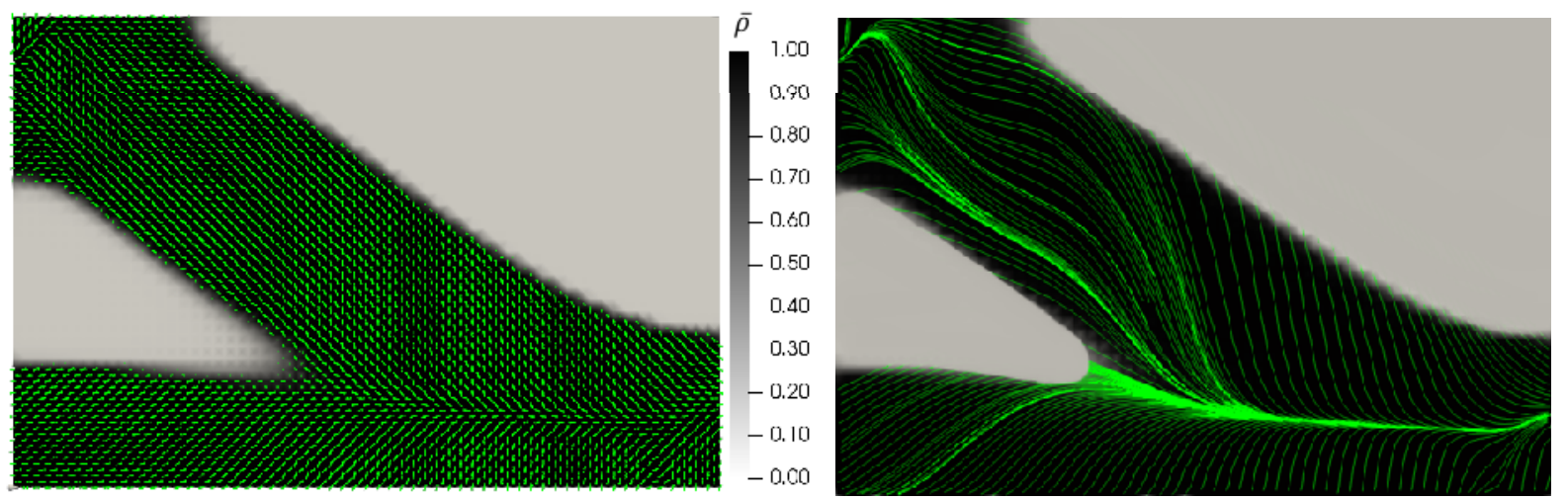

(a) Orientação de fibras e distribuição de material otimizadas para a viga MBB

(b) Interpretação do resultado para a viga MBB

Figura 6.20 - Resultado para a viga MBB

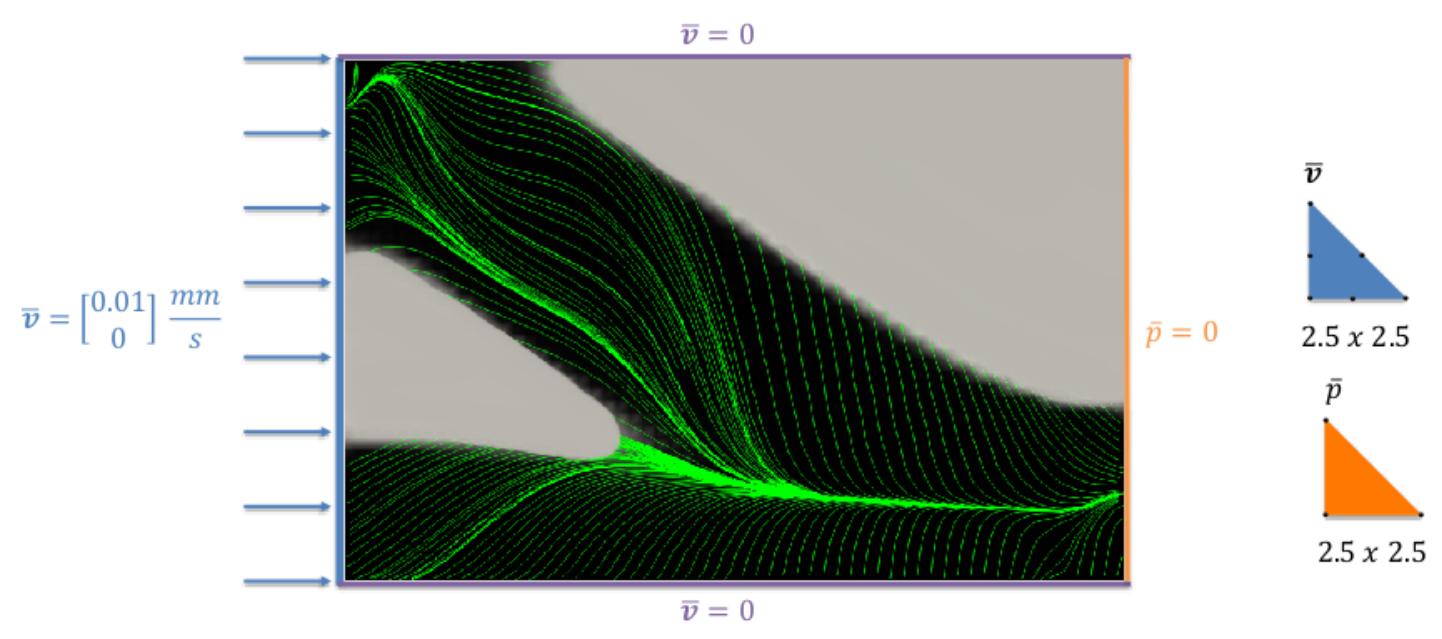

Figura 6.21 - Condições de contorno para o modelo de Borrvall no problema da viga MBB.

\subsubsection{Estrutura em "L"}

O último exemplo é de um estrutura "L" apresentada na Seção 6.2.3. A distribuição inicial de pseudo-densidades considerada, assim nos exemplos anteriores (Seções 6.2.1 


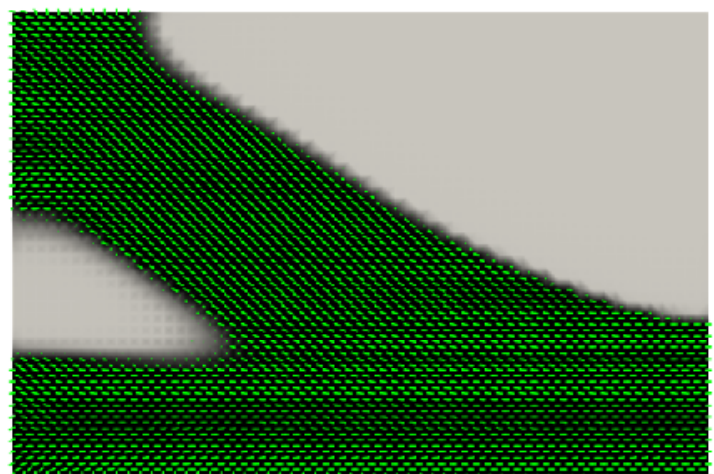

(a) Resultado otimizado com restrição de continuidade.

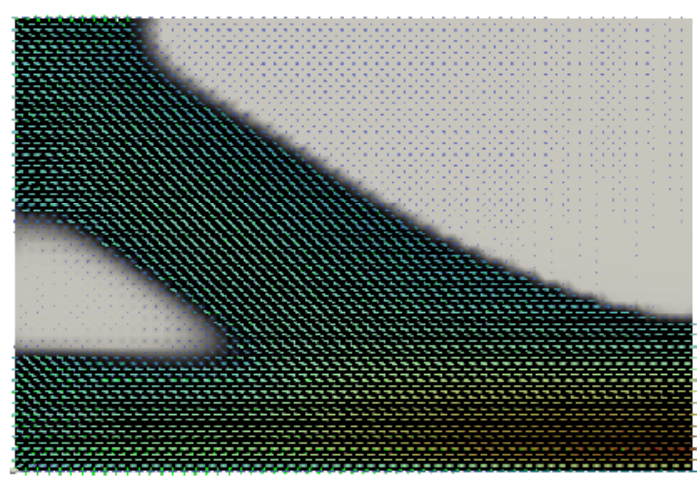

(c) Comparação entre velocidades e fibras.

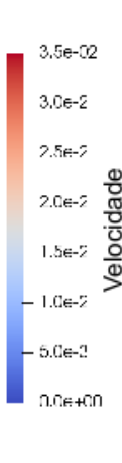

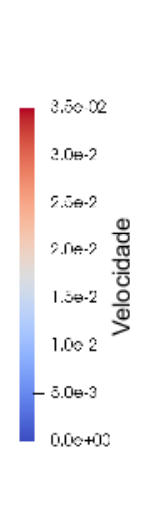

(d)

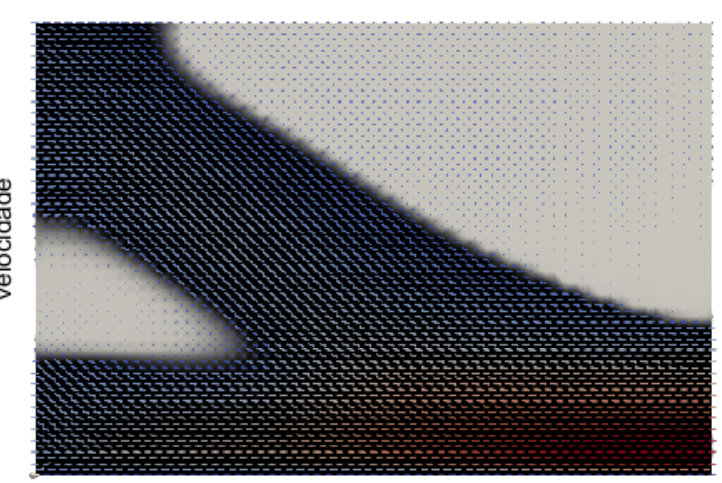

(b) Campo de velocidades

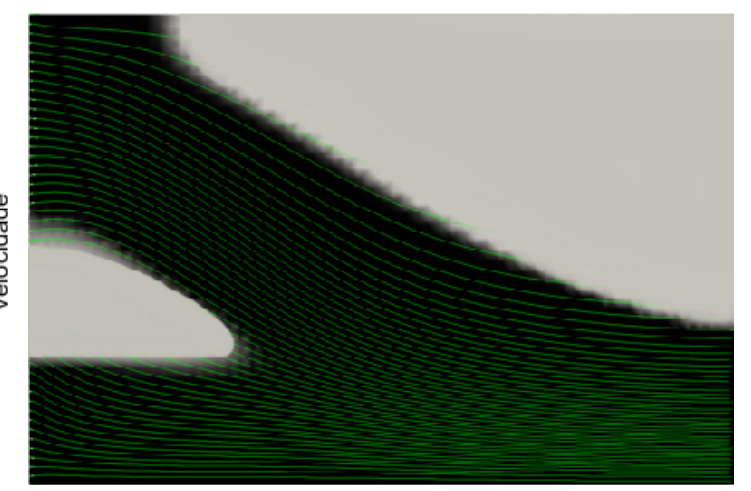

(d) Interpretação do resultado com restrição de continuidade.

Figura 6.22 - Resultado para a viga MBB com restrição de continuidade.

e 6.2.2), é $(\bar{\rho}=0,6)$. Já a distribuição inicial de fibras é feita de forma randômica, para evitar mínimos locais que ocorrem quando considera-se $\theta_{0}=0^{\circ}$ ou $\theta_{0}=90^{\circ}$. Os parâmetros para o filtro de Helmholtz utilizados são $1 \cdot 10^{-5}$ e 1 para $r_{\rho}$ e $r_{\theta}$ respectivamente.

Nos exemplos anteriores (Seções 6.2.2 e 6.2.3), a distribuição de pseudo-densidades $(\bar{\rho})$ na iteração 800 apresentam pouca presença de cinza, porém, para o problema da estrutura em "L", é necessário acrescentar uma regularização à função objetivo em virtude da grande presença de regiões cinzas no domínio. Para este problema a função objetivo passa a ser definida de acordo com a Eq. (6.3)

$$
J=W_{e x t}+w_{r} \int_{V} \rho \cdot(1-\rho) d V
$$

onde $w_{r}$ é um peso para a regularização, o qual é determinado baseado-se no trabalho de Salas (2017) (ver Eq. (6.4))

$$
w_{r, i}=\max \left(w_{r}^{\min }, \min \left(w_{r, i-1} \frac{J_{i}}{J_{i-1}}, w_{r}^{\max }\right)\right)
$$




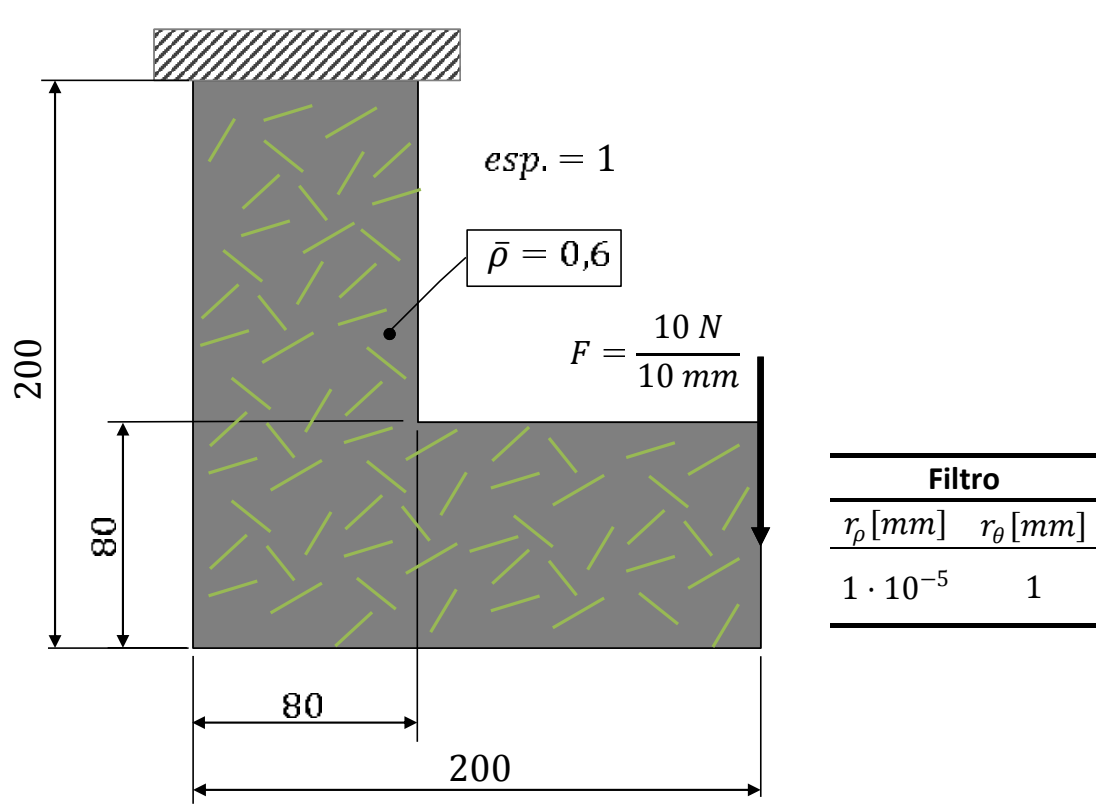

Figura 6.23 - Domínio da estrutura "L"

$\mathrm{Na}$ Eq. (6.4), $i$ representa a iteração atual e $w_{r}^{\min }$ e $w_{r}^{\max }$ são os valores mínimo e máximo para $w_{r}$ respectivamente. Inicialmente, o peso da regularização é $w_{r, 0}=1 \cdot 10^{-2}$. Os valores de $w_{r}^{\min }$ e $w_{r}^{\max }$ são definidos de acordo com a Eq. (6.5)

$$
\begin{aligned}
& w_{r}^{\text {min }}= \begin{cases}0,8 w_{r, 0} & \text { se } i<i_{\text {Borrvall }} \\
1 \cdot 10^{4} w_{r, 0} & \text { se } i \geq i_{\text {Borrvall }}\end{cases} \\
& w_{r}^{\text {max }}= \begin{cases}1 \cdot 10^{4} w_{r, 0} & \text { se } i<i_{\text {Borrvall }} \\
1 \cdot 10^{8} w_{r, 0} & \text { se } i \geq i_{\text {Borvvall }}\end{cases}
\end{aligned}
$$

Para obter uma distribuição de material satisfatória, é necessário utilizar uma continuação onde $i_{\text {max }}^{M I A F I B}$ e $i_{\max }^{S I M P}$ são ambos iguais a 600 , que é também a iteração onde a restrição de continuidade começa a ser considerada. Neste exemplo, a função objetivo é avaliada 800 vezes.

São apresentados na Fig. 6.24 o resultado obtido após 600 iterações, com uma orientação de fibras em cada nó da malha de elementos finitos (ver Fig. 6.24a) e uma possível interpretação para este resultado (ver Fig. 6.24b).

Pode-se verificar por meio da interpretação demonstrada na Fig. 6.24b a existência de mudanças abruptas nas direções e acumulo das fibras em determinadas regiões do domínio. Para tratar estes problemas, a restrição de continuidade é utilizada considerando os mesmos parâmetros utilizados anteriormente (ver Seções 6.2.1 e 6.2.2) e as condições de contorno apresentadas na Fig. 6.25. 

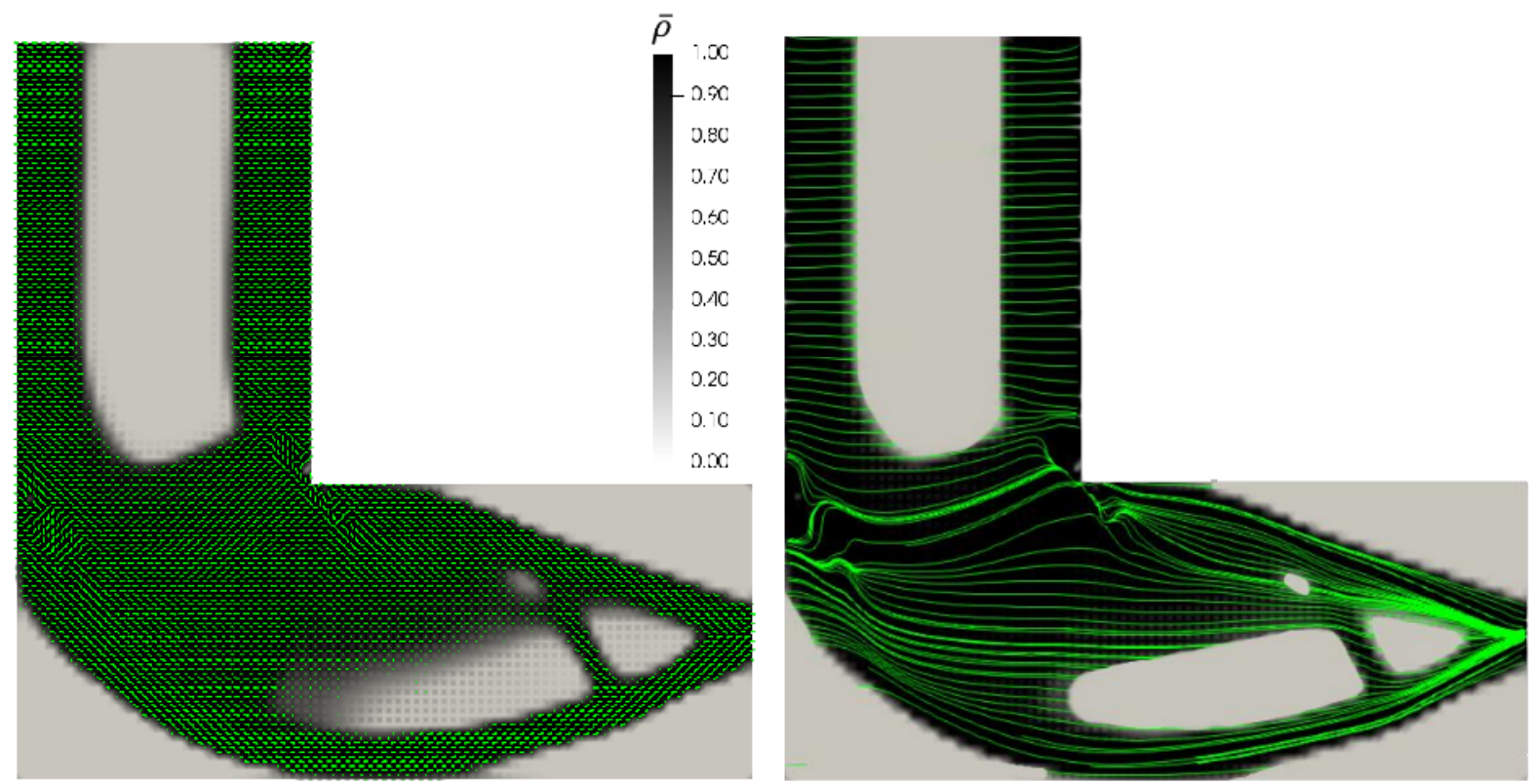

(a) Orientação de fibra e distribuição de material (b) Interpretação do resultado para a estrutura em "L". otimizadas para a estrutura em "L".

Figura 6.24 - Resultado para a estrutura em "L".

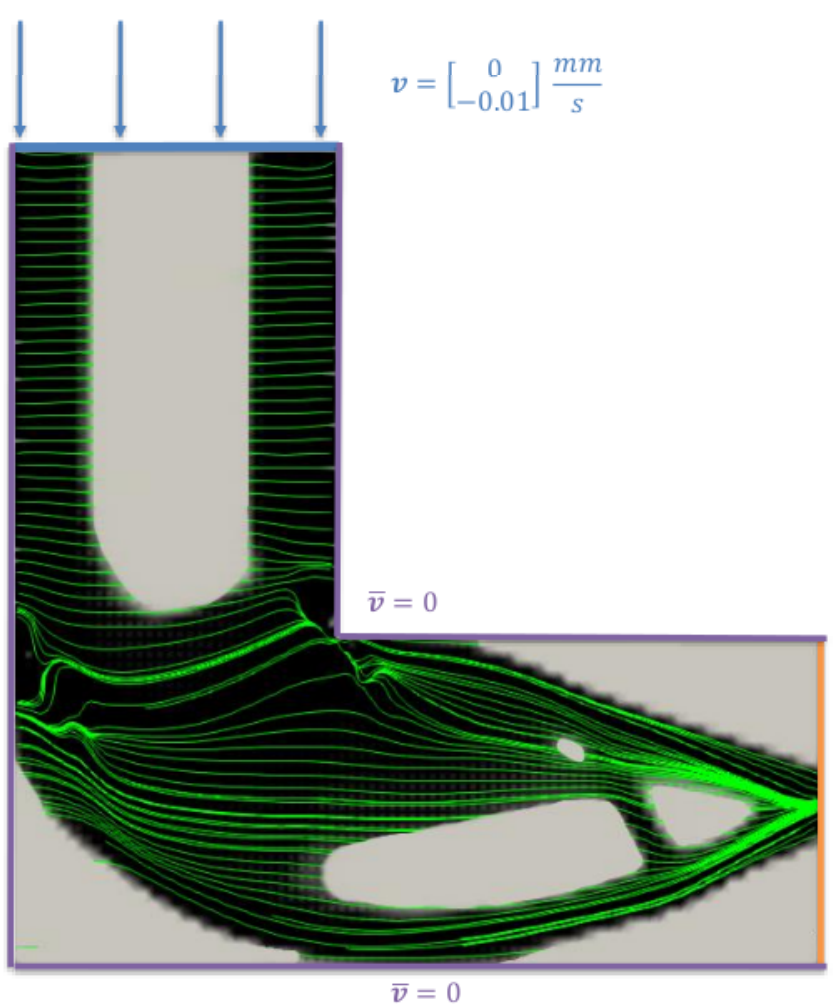

$\bar{p}=0$

Figura 6.25 - Condições de contorno para o modelo de Borrvall no problema da estrutura "L"

O resultado para a estrutura "L" considerando a restrição de continuidade é apresentado na Fig. 6.26a, onde uma orientação de fibra é apresentada em cada nó da malha de 
elementos finitos e o campo de velocidades obtido por meio do modelo de Borrvall é apresentado na Fig. 6.26a. Já a Fig. 6.26c demonstra uma comparação entre o ângulo das fibras e as velocidades, onde é possível observar que na maior parte do domínio, ambos possuem direções similares, com exceções nas fronteiras onde as condições de não escorregamento para o modelo de Borrvall são impostas e em duas regiões no interior do domínio (ver detalhes ampliados na Fig. 6.26c). Uma possível interpretação para o resultado da estrutura "L" é apresentada na Fig. 6.26d, onde, assim como no exemplos anteriores (ver Seções 6.2.1 e 6.2.2), o campo de velocidades obtido é pós-processado para representar as fibras longas. Novamente, observa-se a diminuição das mudanças abruptas na direção das fibras e do acumulo de fibras.

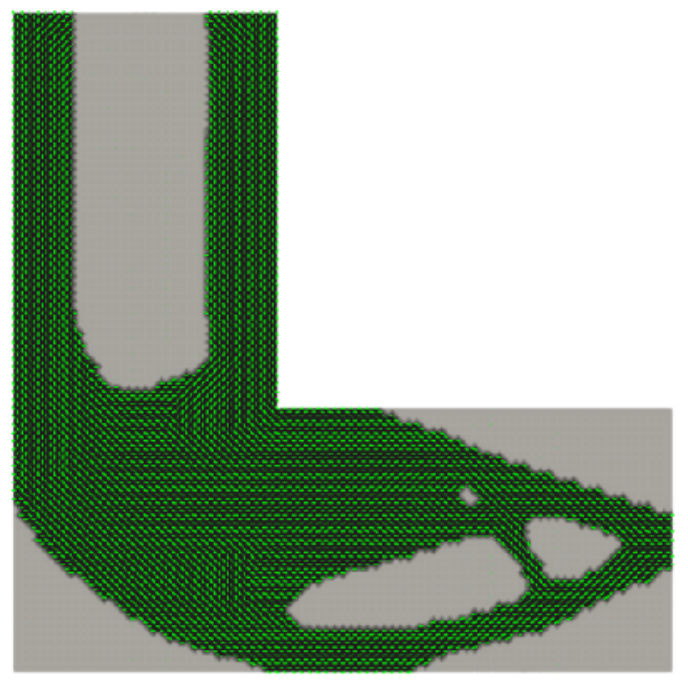

(a) Resultado otimizado com restrição de continuidade.

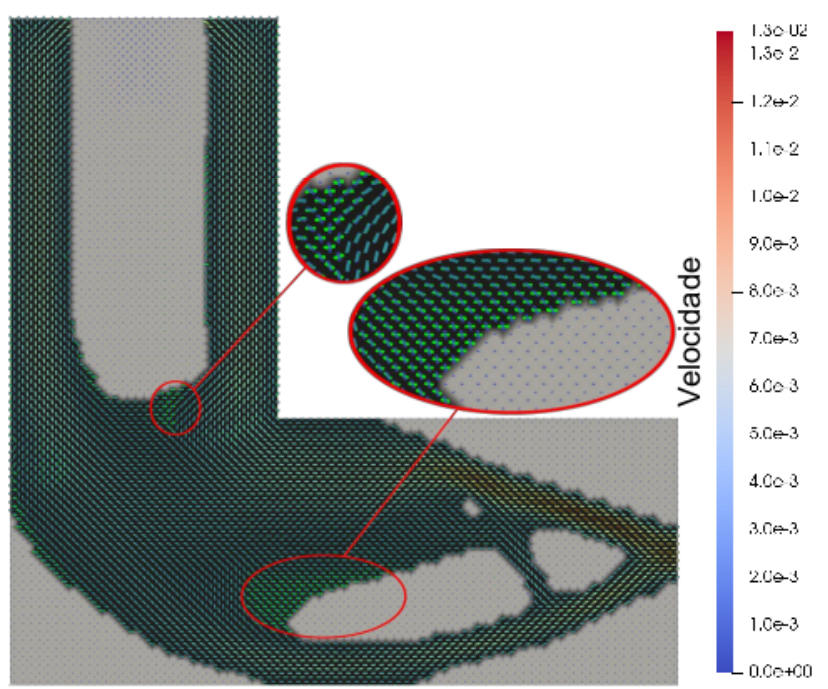

(c) Comparação entre velocidades e fibras.

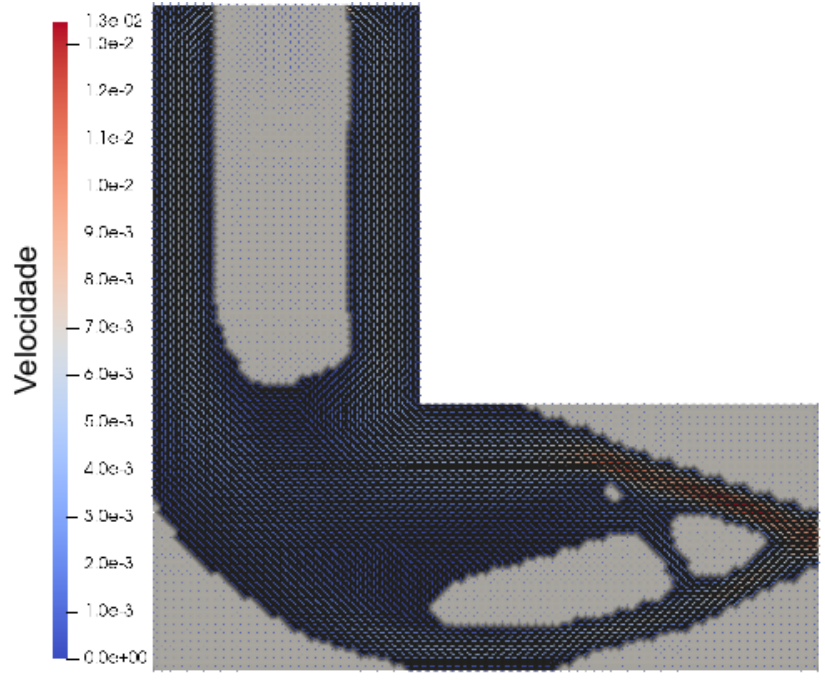

(b) Campo de velocidades.

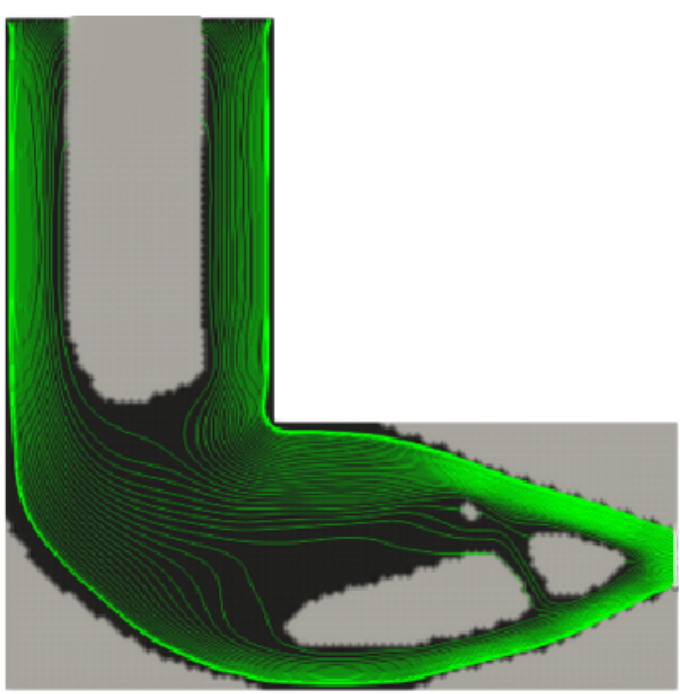

(d) Interpretação do resultado com restrição de continuidade.

Figura 6.26 - Resultado para a estrutura "L" com restrição de continuidade. 


\subsubsection{Convergências das funções objetivo}

Na Fig. 6.27 são apresentadas em um mesmo gráfico as convergências das funções objetivo dos três exemplos da Seção 6.2. As convergências para os problema da viga em balanço e da viga MBB (ver Seções 6.2.1 e 6.2.2) apresentam comportamentos similares. Pode-se observar para estes dois exemplos, um aumento no valor da função objetivo na iteração 800 , onde a restrição de continuidade começa a ser considerada. Para ambos os casos, mesmo após 1000 iterações, não há estabilidade na convergência, no entanto, uma comparação entre os valores das funções objetivo na iteração 800 com a média das últimas 100 iterações é realizada. Para o caso da viga em balanço, a função objetivo na iteração 800 é de 2, 7804 e para as últimas iterações este valor passa a ser 2,8418 $\pm 0,19$, o que representa um aumento médio de $2,21 \%$. Já no caso da viga MBB, este aumento é de $13,1 \%$ (0, 0711 na iteração 800 e 0, $0805 \pm 0,004$ nas última iterações).

A convergência para o problema da estrutura em "L" apresentou valores de função objetivo negativos (ver Fig. 6.28) e, em alguns casos (iterações 76, 173, 325, 409 e 451), iguais a NaN (Not a Number), porém, as iterações onde estes casos ocorreram foram omitidos na Fig. 6.27, por esse motivo, o aumento no valor da função objetivo ocorre antes da iteração 600 e a curva termina antes da iteração 800. Pode-se observar que para este exemplo, houve estabilidade na convergência, no entanto, em função do aumento do peso da regularização após a iteração 600 (ver Eqs. (6.4) e (6.5)) e da presença de cinza (ver Eq. (6.3) e Fig. 6.26), o valor da função objetivo aumenta 4762 vezes se comparado aos valores da iteração antes de considerar a restrição de continuidade. 


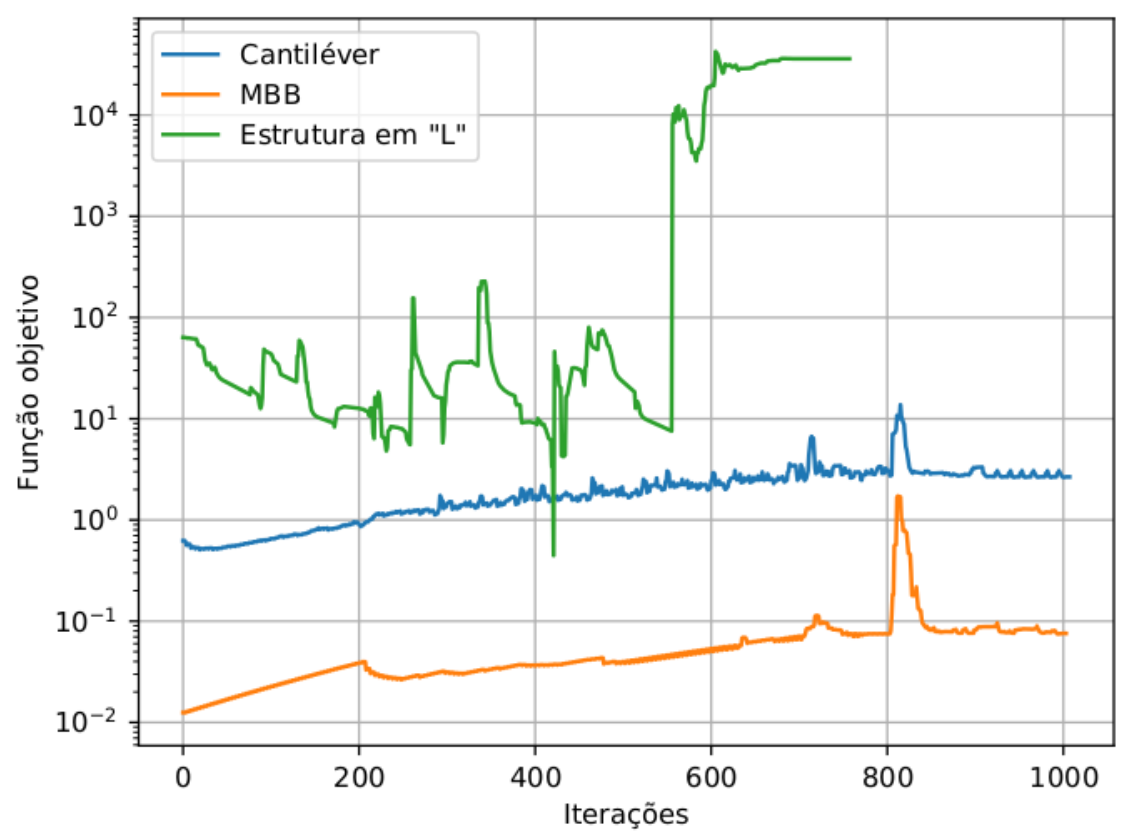

Figura 6.27 - Convergências das funções objetivo.

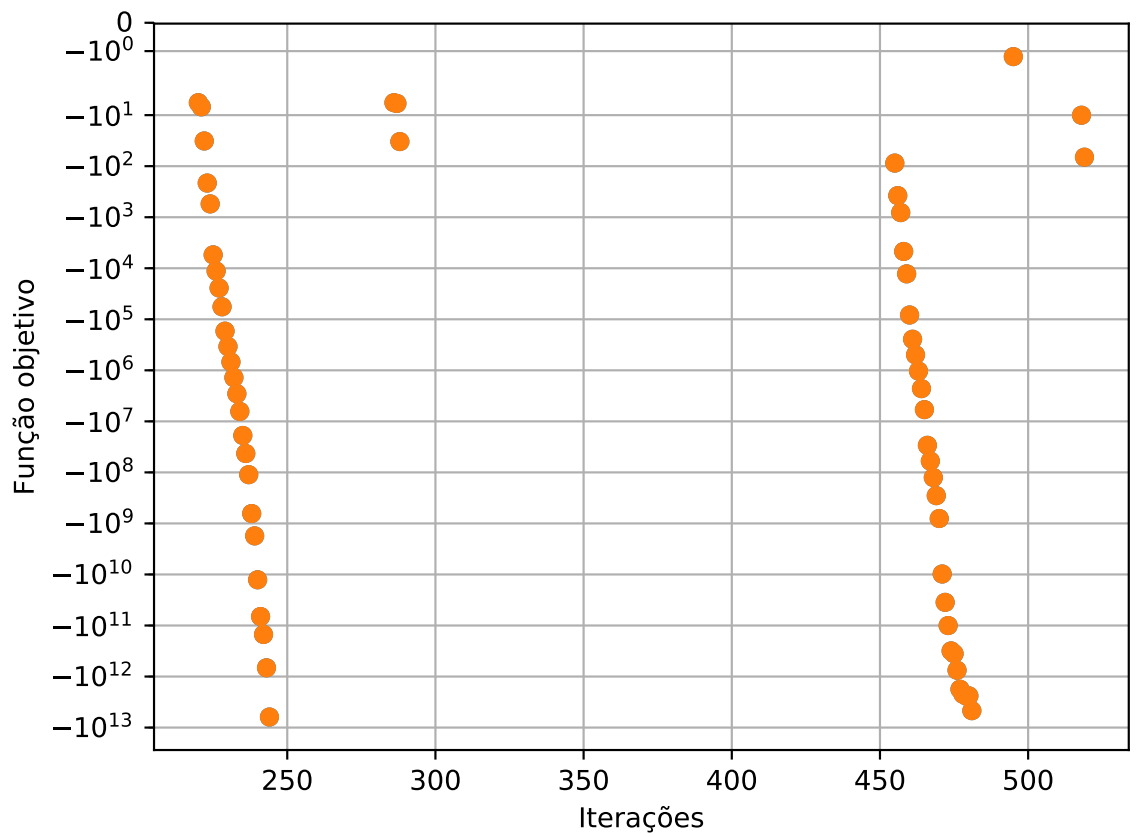

Figura 6.28 - Iterações com valores de função objetivo negativos no exemplo da estrutura "L" 


\section{CONCLUSÕES}

Utilizando a OT, foram realizados projetos de estruturas compósitas reforçadas com fibras considerando um modelo onde ângulos candidatos são definidos a priori (OFDN-m) e um modelo onde o ângulo das fibras é considerado diretamente como uma variável de projeto (MIAFIB).

Duas abordagens foram consideradas. Primeiramente, apenas a distribuição das fibras foi considerada e em uma segunda abordagem, a distribuição otimizada de material no domínio foi realizada simultaneamente à determinação otimizada na orientação das fibras. Os resultados obtidos onde só a distribuição da fibra é considerada mostraram-se condizentes com resultados de problemas similares encontrados na literatura (ver Seções 6.1.1 e 6.1 .3 e (KIYONO; SILVA; REDDY, 2017)).

O filtro proposto por (LAZAROV; SIGMUND, 2011), que utiliza uma equação de Helmholtz modificada, provou ser uma possível abordagem para tratar a descontinuidade das fibras. Com o aumento do valor do parâmetro $r$ consegue-se uma melhor continuidade nas fibras, ou seja, mudanças bruscas nas direções das fibras, que podem causar problemas como concentração de tensão, são mitigadas.

A restrição de continuidade baseada no modelo de Borrvall garantiu a continuidade das fibras e mitigou o problema de acumulo das mesmas, provendo resultados mais viáveis para a manufatura.

A suavização na continuidade das fibras, em geral, provoca um aumento no valor final da função objetivo. A imposição da continuidade, realizada por meio do filtro de Helmholtz e por meio da restrição de continuidade, diminui o espaço de solução, tornando as soluções mais restritas.

A implementação realizada no ambiente FEniCS combinado com o pacote Dolfin-Adjoint, mostrou-se uma abordagem eficaz para a solução do problema de direcionamento das fibras e o método do ponto interior, implementado no IPOpt, provou-se eficiente para os problemas de otimização solucionados no Capítulo 6.

\subsection{Atividades futuras}

Os resultados deste trabalho foram obtidos utilizando elementos sólidos e planos. Como trabalho futuro, pretende-se realizar otimizações utilizando elementos de placas e cascas, para que a otimização seja realizada em compósitos com múltiplas camadas, onde outras restrições de manufatura, além da restrição de continuidade, serão consideradas. 
Neste trabalho as hipóteses de pequenos deslocamentos, deformações e rotações foram adotadas e em razão disto, foi possível utilizar uma equação constitutiva linear. Como extensão deste trabalho, pretende-se utilizar a equação constitutiva proposta por Bonet e Burton (1998), que possibilita a modelagem de materiais ortotrópicos considerando grandes deformações. 


\section{REFERÊNCIAS}

ALMEIDA, S. F. M. Mecânica de Estruturas de Materiais Compósitos. 2018. Cited 2 times in pages 31 e 32 .

ALNÆS, M. S. et al. Unified form language: A domain-specific language for weak formulations of partial differential equations. ACM Trans. Math. Softw., ACM, New York, NY, USA, v. 40, n. 2, p. 9:1-9:37, mar. 2014. ISSN 0098-3500. Available from Internet: $<$ http://doi.acm.org/10.1145/2566630>. Cited in page 35.

ANTÓNIO, C. C.; BARBOSA, J. T.; DINIS, L. S. Optimal design of beam reinforced composite structures under elasto-plastic loading conditions. Structural and Multidisciplinary Optimization, v. 19, n. 1, p. 50-63, Mar 2000. ISSN 1615-1488. Available from Internet: $<$ https://doi.org/10.1007/s001580050085>. Cited in page 21.

ARORA, J. S. Introduction to optimum design. [S.I.]: Elsevier, 2004. Cited 2 times in pages 61 e 68.

AWAD, Z. K. et al. A review of optimization techniques used in the design of fibre composite structures for civil engineering applications. Materials \& Design, Elsevier, v. 33, p. 534-544, 2012. Cited in page 20.

AYACHIT, U. The paraview guide: a parallel visualization application. [S.I.]: Kitware, Inc., 2015. Cited in page 82.

AZARAFZA, R. et al. Analysis and optimization of laminated composite circular cylindrical shell subjected to compressive axial and transverse transient dynamic loads. Thin-Walled Structures, v. 47, n. 8, p. 970 - 983, 2009. ISSN 0263-8231. Available from Internet: <http://www.sciencedirect.com/science/article/pii/S026382310900024X>. Cited in page 20.

BATHE, K.-J. Finite element procedures. [S.I.]: Klaus-Jurgen Bathe, 2006. Cited 7 times in pages 33, 34, 35, 36, 37, 103 e 104.

BAZARAA, M. S.; SHERALI, H. D.; SHETTY, C. M. Nonlinear programming: theory and algorithms. [S.I.]: John Wiley \& Sons, 2013. Cited in page 61.

BENDS $\varnothing E$, M. P. Optimal shape design as a material distribution problem. Structural optimization, v. 1, n. 4, p. 193-202, Dec 1989. ISSN 1615-1488. Available from Internet: $<$ https://doi.org/10.1007/BF01650949>. Cited 2 times in pages 23 e 39.

BENDSØE, M. P.; KIKUCHI, N. Generating optimal topologies in structural design using a homogenization method. Computer methods in applied mechanics and engineering, Elsevier, v. 71, n. 2, p. 197-224, 1988. Cited in page 39.

BENDSØE, M. P.; SIGMUND, O. Optimization of structural topology, shape, and material. [S.I.]: Springer, 1995. v. 414. Cited in page 45.

BENDSØE, M. P.; SIGMUND, O. Topology optimization: theory, methods and applications. 2003. [S.I.]: Springer, 2003. Cited 7 times in pages 40, 41, 43, 45, 46, 62 e 81. 
BODDINGTON, C. E.; RANDALL, W. C. Nonlinear programs for product blending. Joint National TIMS/ORSA Meeting, p. 151-164, 1979. Cited in page 68.

BONET, J.; BURTON, A. A simple orthotropic, transversely isotropic hyperelastic constitutive equation for large strain computations. Computer methods in applied mechanics and engineering, Elsevier, v. 162, n. 1-4, p. 151-164, 1998. Cited 2 times in pages 25 e 94.

BORRVALL, T.; PETERSSON, J. Topology optimization of fluids in stokes flow. International journal for numerical methods in fluids, Wiley Online Library, v. 41, n. 1, p. 77-107, 2003. Cited 4 times in pages 53, 54, 55 e 56.

BOURDIN, B. Filters in topology optimization. International Journal for Numerical Methods in Engineering, v. 50, n. 9, p. 2143-2158, 2001. Available from Internet: $<$ https://onlinelibrary.wiley.com/doi/abs/10.1002/nme.116>. Cited in page 46.

BRUNS, T. E.; TORTORELLI, D. A. Topology optimization of non-linear elastic structures and compliant mechanisms. Computer methods in applied mechanics and engineering, Elsevier, v. 190, n. 26-27, p. 3443-3459, 2001. Cited in page 46.

BRUYNEEL, M. Sfp-a new parameterization based on shape functions for optimal material selection: application to conventional composite plies. Structural and Multidisciplinary Optimization, Springer, v. 43, n. 1, p. 17-27, 2011. Cited in page 21.

BUCALEM, M. L.; BATHE, K.-J. The mechanics of solids and structures-hierarchical modeling and the finite element solution. [S.I.]: Springer Science \& Business Media, 2011. Cited 3 times in pages 33, 35 e 36.

BUHL, T.; PEDERSEN, C. B.; SIGMUND, O. Stiffness design of geometrically nonlinear structures using topology optimization. Structural and Multidisciplinary Optimization, Springer, v. 19, n. 2, p. 93-104, 2000. Cited in page 39.

BUTYRIN, V. I. et al. Application of the coordinatewise descent method on a unit interval for weight optimization of structures made of composite materials. Journal of Applied Mechanics and Technical Physics, v. 50, n. 4, p. 720-727, Jul 2009. ISSN 1573-8620. Available from Internet: <https://doi.org/10.1007/s10808-009-0098-7>. Cited in page 21.

BUZBY, B. Techniques and experience solving really big nonlinear programs. Optimization Methods. English Universities Press, 1974. Cited in page 68.

CHEN, B.-C.; KIKUCHI, N. Topology optimization with design-dependent loads. Finite Elements in Analysis and Design, Elsevier, v. 37, n. 1, p. 57-70, 2001. Cited in page 40.

$\mathrm{CHO}, \mathrm{H}$. Optimization of dynamic behaviors of an orthotropic composite shell subjected to hygrothermal environment. Finite Elements in Analysis and Design, v. 45 , n. 11 , p. $852-860,2009$. ISSN 0168-874X. Available from Internet: <http://www.sciencedirect.com/science/article/pii/S0168874X09000985>. Cited in page 20.

DANIEL, I. M. et al. Engineering mechanics of composite materials. [S.I.]: Oxford university press New York, 1994. v. 3. Cited 5 times in pages 18, 25, 26, 27 e 29.

DEVECI, H. A.; AYDIN, L.; ARTEM, H. S. Buckling optimization of composite laminates using a hybrid algorithm under puck failure criterion constraint. Journal of Reinforced Plastics and Composites, v. 35, n. 16, p. 1233-1247, 2016. Available from Internet: <https://doi.org/10.1177/0731684416646860>. Cited in page 20. 
DIAZ, A.; SIGMUND, O. Checkerboard patterns in layout optimization. Structural optimization, Springer, v. 10, n. 1, p. 40-45, 1995. Cited in page 43.

DILLINGER, J. et al. Stiffness optimization of composite wings with aeroelastic constraints. Journal of Aircraft, American Institute of Aeronautics and Astronautics, v. 50, n. 4, p. 1159-1168, 2013. Cited in page 20.

DUYSINX, P. Layout Optimization : A Mathematical Programming Approach. 1997. Cited in page 45.

FUNKE, S. The automation of PDE-constrained optimisation and its applications. Tese (Doutorado), 11 2013. Cited 3 times in pages 64, 65 e 67.

FUNKE, S. W.; FARRELL, P. E. A framework for automated pde-constrained optimisation. CoRR, abs/1302.3894, 2013. Available from Internet: <http://arxiv.org/abs/1302.3894>. Cited in page 67.

GAO, T.; ZHANG, W.; DUYSINX, P. A bi-value coding parameterization scheme for the discrete optimal orientation design of the composite laminate. International Journal for Numerical Methods in Engineering, Wiley Online Library, v. 91, n. 1, p. 98-114, 2012. Cited in page 21.

GEA, H.; LUO, J. On the stress-based and strain-based methods for predicting optimal orientation of orthotropic materials. Structural and Multidisciplinary Optimization, Springer, v. 26, n. 3-4, p. 229-234, 2004. Cited in page 73.

GRIFFITH, R. E.; STEWART, R. A. A nonlinear programming technique for the optimization of continuous processing systems. Management Science, v. 7, n. 4, p. 379-392, 1961. Available from Internet: <https://doi.org/10.1287/mnsc.7.4.379>. Cited in page 68.

HABER, R. B.; JOG, C. S.; BENDSØE, M. P. A new approach to variable-topology shape design using a constraint on perimeter. Structural optimization, v. 11, n. 1, p. 1-12, Feb 1996. ISSN 1615-1488. Available from Internet: <https://doi.org/10.1007/BF01279647>. Cited in page 45.

HAMBACH, M.; RUTZEN, M.; VOLKMER, D. Chapter 5 - properties of 3d-printed fiber-reinforced portland cement paste. In: SANJAYAN, J. G.; NAZARI, A.; NEMATOLLAHI, B. (Ed.). 3D Concrete Printing Technology. Butterworth-Heinemann, 2019. p. $73-113$. ISBN 978-0-12-815481-6. Available from Internet: <http://www.sciencedirect.com/science/ article/pii/B9780128154816000051>. Cited 2 times in pages 19 e 20.

HERATH, M. T. et al. Structural strength and laminate optimization of self-twisting composite hydrofoils using a genetic algorithm. Composite Structures, v. 176, p. $359-378,2017$. ISSN 0263-8223. Available from Internet: <http://www.sciencedirect.com/science/article/pii/ S0263822316323443>. Cited in page 20.

HOU, Z. et al. 3d printed continuous fibre reinforced composite corrugated structure. Composite Structures, Elsevier, v. 184, p. 1005-1010, 2018. Cited in page 18.

IKEYA, K.; SHIMODA, M.; SHI, J.-X. Multi-objective free-form optimization for shape and thickness of shell structures with composite materials. Composite Structures, v. 135, p. $262-275,2016$. ISSN 0263-8223. Available from Internet: <http://www.sciencedirect.com/science/article/pii/S0263822315008478>. Cited in page 21. 
JAFARI, M.; ROHANI, A. Optimization of perforated composite plates under tensile stress using genetic algorithm. Journal of Composite Materials, v. 50, n. 20, p. 2773-2781, 2016. Available from Internet: <https://doi.org/10.1177/0021998315612540>. Cited in page 20.

JENSEN, J. S.; SIGMUND, O. Systematic design of photonic crystal structures using topology optimization: Low-loss waveguide bends. Applied physics letters, AIP, v. 84, n. 12, p. 2022-2024, 2004. Cited in page 39.

JOG, C. S.; HABER, R. B. Stability of finite element models for distributed-parameter optimization and topology design. Computer methods in applied mechanics and engineering, Elsevier, v. 130, n. 3-4, p. 203-226, 1996. Cited in page 43.

JOG, C. S.; HABER, R. B.; BENDSøE, M. P. Topology design with optimized, self-adaptive materials. International Journal for Numerical Methods in Engineering, Wiley Online Library, v. 37, n. 8, p. 1323-1350, 1994. Cited in page 43.

KAW, A. K. Mechanics of composite materials. [S.I.]: CRC press, 2005. Cited 11 times in pages 18, 25, 26, 27, 28, 29, 30, 31, 32, 70 e 79.

KHARMANDA, G. et al. Reliability-based topology optimization. Structural and Multidisciplinary Optimization, Springer, v. 26, n. 5, p. 295-307, 2004. Cited in page 40.

KIM, D.-H.; KIM, H.-G.; KIM, H.-S. Design optimization and manufacture of hybrid glass/carbon fiber reinforced composite bumper beam for automobile vehicle. Composite Structures, v. 131, p. 742 - 752, 2015. ISSN 0263-8223. Available from Internet: <http://www.sciencedirect.com/science/article/pii/S0263822315004924>. Cited in page 20.

KIYONO, C.; SILVA, E.; REDDY, J. A novel fiber optimization method based on normal distribution function with continuously varying fiber path. Composite Structures, Elsevier, v. 160, p. 503-515, 2017. Cited 8 times in pages 21, 23, 48, 49, 50, 61, 62 e 93.

KUHN, H. W.; TUCKER, A. W. Nonlinear programming. In: Proceedings of the Second Berkeley Symposium on Mathematical Statistics and Probability. Berkeley, Calif.: University of California Press, 1951. p. 481-492. Available from Internet: <https://projecteuclid.org/euclid.bsmsp/1200500249>. Cited in page 61.

LAI, W. M. et al. Introduction to continuum mechanics. [S.I.]: Butterworth-Heinemann, 2009. Cited 2 times in pages 26 e 27.

LANGTANGEN, H. P.; LOGG, A. Solving PDEs in minutes-the FEniCS tutorial volume $i$. [S.I.]: Springer, 2017. Cited in page 66.

LAU, G. K.; DU, H.; LIM, M. K. Techniques to suppress intermediate density in topology optimization of compliant mechanisms. Computational Mechanics, v. 27 , n. 5, p. 426-435, May 2001. ISSN 1432-0924. Available from Internet: $<$ https://doi.org/10.1007/s004660100255>. Cited in page 41.

LAZAROV, B. S.; SIGMUND, O. Filters in topology optimization based on helmholtz-type differential equations. International Journal for Numerical Methods in Engineering, Wiley Online Library, v. 86, n. 6, p. 765-781, 2011. Cited 7 times in pages 23, 46, 53, 61, 62, 93 e 105.

MAUTE, K.; RAMM, E. Adaptive topology optimization of shell structures. AIAA journal, v. 35, n. 11 , p. 1767-1773, 1997. Cited in page 40. 
MAUTE, K.; SCHWARZ, S.; RAMM, E. Adaptive topology optimization of elastoplastic structures. Structural optimization, Springer, v. 15, n. 2, p. 81-91, 1998. Cited in page 40.

MEJLEJ, V. G. et al. Optimization of variable stiffness composites in automated fiber placement process using evolutionary algorithms. Procedia CIRP, v. 66, p. $79-84,2017$. ISSN 2212-8271. 1st CIRP Conference on Composite Materials Parts Manufacturing (CIRP CCMPM 2017). Available from Internet: <http://www.sciencedirect.com/science/article/pii/ S2212827117304900>. Cited in page 20.

MOITA, J. M. S. et al. Optimal design in vibration control of adaptive structures using a simulated annealing algorithm. Composite Structures, Elsevier, v. 75, n. 1-4, p. 79-87, 2006. Cited in page 20.

MOITA, J. S. et al. Optimal dynamic control of laminated adaptive structures using a higher order model and a genetic algorithm. Computers \& Structures, v. 86, n. 3, p. 198 - 206, 2008. ISSN 0045-7949. Smart Structures. Available from Internet: <http://www.sciencedirect.com/science/article/pii/S0045794907000582>. Cited in page 20.

NICHOLAS, P. E.; PADMANABAN, K.; SOFIA, A. S. Optimization of dispersed laminated composite plate for maximum safety factor using genetic algorithm and various failure criteria. Procedia Engineering, v. 38, p. 1209 - 1217, 2012. ISSN 1877-7058. INTERNATIONAL CONFERENCE ON MODELLING OPTIMIZATION AND COMPUTING. Available from Internet: <http://www.sciencedirect.com/science/article/pii/S1877705812020656>. Cited in page 20.

NIKBAKT, S.; KAMARIAN, S.; SHAKERI, M. A review on optimization of composite structures part i: Laminated composites. Composite Structures, Elsevier, 2018. Cited in page 20.

NING, F. et al. Additive manufacturing of carbon fiber-reinforced plastic composites using fused deposition modeling: Effects of process parameters on tensile properties. Journal of Composite Materials, SAGE Publications Sage UK: London, England, v. 51, n. 4, p. 451-462, 2017. Cited in page 18.

NING, F. et al. Additive manufacturing of carbon fiber reinforced thermoplastic composites using fused deposition modeling. Composites Part B: Engineering, Elsevier, v. 80, p. 369-378, 2015. Cited in page 18.

NOCEDAL, J.; WRIGHT, S. Numerical optimization. [S.I.]: Springer Science \& Business Media, 2006. Cited 2 times in pages 61 e 68.

OLHOFF, N.; DU, J. Topology optimization of vibrating bi-material structures with respect to sound radiation. In: BENDS $\varnothing \mathrm{E}, \mathrm{M}$. P.; OLHOFF, N.; SIGMUND, O. (Ed.). IUTAM Symposium on Topological Design Optimization of Structures, Machines and Materials. Dordrecht: Springer Netherlands, 2006. p. 43-52. ISBN 978-1-4020-4752-7. Cited in page 40.

PETERSSON, J.; SIGMUND, O. Slope constrained topology optimization. International Journal for Numerical Methods in Engineering, v. 41, n. 8, p. 1417-1434, 1998. Available from Internet: <https://onlinelibrary.wiley.com/doi/abs/10.1002/\%28SICI\%291097-0207\% $2819980430 \% 2941 \% 3 A 8 \% 3 C 1417 \% 3 A \% 3 A A I D-N M E 344 \% 3 E 3.0 . C O \% 3 B 2-N>$. Cited in page 46. 
PIMENTA, P. d. M. Fundamentos da mecânica dos sólidos e das estruturas. Escola Politécnica da Universidade de Sao Paulo, 2008. Cited 3 times in pages 26, 27 e 35.

QIU, J. B.; ZHU, X. The buckling optimization on composite beam with hat stiffener. In: TRANS TECH PUBL. Advanced Materials Research. [S.I.], 2014. v. 953, p. 1649-1652. Cited in page 20.

QUAN, Z. et al. Additive manufacturing of multi-directional preforms for composites: opportunities and challenges. Materials Today, Elsevier, v. 18, n. 9, p. 503-512, 2015. Cited in page 18.

ROZVANY, G. I. Structural design via optimality criteria: the Prager approach to structural optimization. [S.I.]: Springer Science \& Business Media, 2012. v. 8. Cited in page 67.

ROZVANY, G. I. Shape and layout optimization of structural systems and optimality criteria methods. [S.I.]: Springer, 2014. v. 325. Cited in page 67.

ROZVANY, G. I.; ZHOU, M.; BIRKER, T. Generalized shape optimization without homogenization. Structural optimization, Springer, v. 4, n. 3-4, p. 250-252, 1992. Cited 2 times in pages 23 e 39.

ROZVANY, G. I. N. A critical review of established methods of structural topology optimization. Structural and Multidisciplinary Optimization, v. 37, n. 3, p. 217-237, Jan 2009. ISSN 1615-1488. Available from Internet: <https://doi.org/10.1007/s00158-007-0217-0>. Cited in page 40.

SÁ, L. F. N. d. Topology optimization method applied to laminar flow machine rotor design. Tese (Doutorado) — Universidade de São Paulo, 2016. Cited 2 times in pages 66 e 67.

SALAS, R. A. Projeto Dinâmico de Estruturas Piezocompósitas Laminadas (EPLA) Utilizando o Método de Otimização Topológica (MOT). Tese (Doutorado) — Universidade de São Paulo, 2017. Cited in page 87.

SALAS, R. A. et al. Optimized dynamic design of laminated piezocomposite multi-entry actuators considering fiber orientation. Computer Methods in Applied Mechanics and Engineering, v. 335, p. 223 - 254, 2018. ISSN 0045-7825. Available from Internet: $<$ http://www.sciencedirect.com/science/article/pii/S0045782518300902>. Cited 5 times in pages 22, 23, 51, 52 e 61 .

SAVE, M. et al. Structural Optimization: Optimality criteria. [S.I.]: Plenum Pub Corporation, 1985. v. 1. Cited in page 67.

SIGMUND, O. On the design of compliant mechanisms using topology optimization. Journal of Structural Mechanics, Taylor \& Francis, v. 25, n. 4, p. 493-524, 1997. Cited in page 39.

SIGMUND, O. Design of multiphysics actuators using topology optimization-part i: One-material structures. Computer methods in applied mechanics and engineering, Elsevier, v. 190 , n. 49-50, p. 6577-6604, 2001. Cited in page 39.

SIGMUND, O. On the usefulness of non-gradient approaches in topology optimization. Structural and Multidisciplinary Optimization, v. 43, n. 5, p. 589-596, May 2011. ISSN 1615-1488. Available from Internet: <https://doi.org/10.1007/s00158-011-0638-7>. Cited 2 times in pages 20 e 21. 
SIGMUND, O.; PETERSSON, J. Numerical instabilities in topology optimization: a survey on procedures dealing with checkerboards, mesh-dependencies and local minima. Structural optimization, Springer, v. 16, n. 1, p. 68-75, 1998. Cited 3 times in pages 44, 45 e 46.

SILVA, A. L. F. et al. Topology optimization of fibers orientation in composite material with hyperelastic matrix. Composite Structures, Elsevier, 2019. Cited in page 49.

SKAVHAUG, O. FEniCS Project - Core components. 2016. [Acessado 30-maio-2019]. Cited in page 66.

SØRENSEN, R.; LUND, E. Thickness filters for gradient based multi-material and thickness optimization of laminated composite structures. Structural and Multidisciplinary Optimization, v. 52, n. 2, p. 227-250, Aug 2015. ISSN 1615-1488. Available from Internet: $<$ https://doi.org/10.1007/s00158-015-1230-3>. Cited in page 21.

STEGMANN, J.; LUND, E. Discrete material optimization of general composite shell structures. International Journal for Numerical Methods in Engineering, Wiley Online Library, v. 62, n. 14, p. 2009-2027, 2005. Cited 3 times in pages 21, 48 e 51.

SUDHAGAR, P. E. et al. Structural optimization of rotating tapered laminated thick composite plates with ply drop-offs. International Journal of Mechanics and Materials in Design, Springer, v. 13, n. 1, p. 85-124, 2017. Cited in page 20.

SVANBERG, K. The method of moving asymptotes-a new method for structural optimization. International journal for numerical methods in engineering, Wiley Online Library, v. 24, n. 2, p. 359-373, 1987. Cited in page 67.

TAYLOR, J. E.; ROSSOW, M. P. Optimal truss design based on an algorithm using optimality criteria. International Journal of Solids and Structures, Elsevier, v. 13, n. 10, p. 913-923, 1977. Cited in page 67.

TRAHAN, D. H. The mixed partial derivatives and the double derivative. The American Mathematical Monthly, Taylor \& Francis, v. 76, n. 1, p. 76-77, 1969. Cited in page 27.

VASILIEV, V. V.; MOROZOV, E. V. Mechanics and analysis of composite materials. [S.I.]: Elsevier, 2001. Cited in page 18.

VENKATARAMAN, S.; HAFTKA, R. T. Optimization of composite panels-a review. In: Proceedings-American Society for Composites. [S.I.: s.n.], 1999. p. 479-488. Cited in page 20.

WÄCHTER, A.; BIEGLER, L. T. On the implementation of an interior-point filter line-search algorithm for large-scale nonlinear programming. Mathematical programming, Springer, v. 106 , n. 1 , p. 25-57, 2006. Cited in page 68.

YIN, L.; ANANTHASURESH, G. Topology optimization of compliant mechanisms with multiple materials using a peak function material interpolation scheme. Structural and Multidisciplinary Optimization, v. 23, n. 1, p. 49-62, Dec 2001. ISSN 1615-1488. Available from Internet: <https://doi.org/10.1007/s00158-001-0165-z>. Cited in page 22.

ZHOU, M.; SHYY, Y.; THOMAS, H. Checkerboard and minimum member size control in topology optimization. Structural and Multidisciplinary Optimization, v. 21 , n. 2, p. 152-158, Apr 2001. ISSN 1615-1488. Available from Internet: $<$ https://doi.org/10.1007/s001580050179>. Cited 3 times in pages 43, 45 e 46. 
ZURAS, D. et al. IEEE standard for floating-point arithmetic. IEEE Std 754-2008, IEEE, p. 1-70, 2008. Cited in page 50. 


\section{APÊNDICE A - IMPLEMENTAÇÃO DO MEF PARA PROBLEMAS ESTRUTURAIS}

Para a aproximar a solução da equação Eq. (2.33) utilizando o MEF, é assumida a hipótese de que o sólido apresentado na Fig. 2.4 pode ser aproximado como um conjunto de elementos finitos discretos interconectados nos nós. Os deslocamentos para cada nó $a$ do elemento $m$ são determinados por meio do produto de uma matriz de interpolação dos deslocamentos $\mathbf{H}^{m}$ por um vetor de deslocamentos em coordenadas globais $\hat{\mathbf{u}}$ (ver Eq. (A.1)) (BATHE, 2006)

$$
\mathbf{u}^{(m)}(x, y, z)=\mathbf{H}^{(m)}(x, y, z) \hat{\mathbf{u}}
$$

A aproximação das deformações para um elemento $m$ é definida por meio do produto da matriz B, que relaciona deformação e deslocamento, pelo vetor î (ver Eq. (A.2)) (BATHE, 2006)

$$
\varepsilon^{(m)}(x, y, z)=\mathbf{B}^{(m)}(x, y, z) \hat{\mathbf{u}}
$$

Os deslocamentos e deformações virtuais, $\delta \mathbf{u}$ e $\delta \varepsilon$ respectivamente, são definidos de forma análoga (ver Eqs. (A.3) e (A.4))

$$
\begin{aligned}
& \delta \mathbf{u}^{(m)}(x, y, z)=\mathbf{H}^{(m)}(x, y, z) \delta \hat{\mathbf{u}} \\
& \delta \varepsilon^{(m)}(x, y, z)=\mathbf{B}^{(m)}(x, y, z) \delta \hat{\mathbf{u}}
\end{aligned}
$$

A solução aproximada para o princípio dos trabalhos virtuais pode ser obtida substituindo 
as Eqs. (A.1) a (A.4) em Eq. (2.33) (ver Eq. (A.5)) (BATHE, 2006).

$$
\begin{aligned}
& \delta \hat{\mathbf{u}}^{T}\left(\sum_{m} \int_{V^{(m)}} \mathbf{B}^{(m)} \mathbf{C}^{(m)} \mathbf{B}^{(m)} d V^{(m)}\right) \hat{\mathbf{u}}= \\
& \delta \hat{\mathbf{u}}^{T}\left(\sum_{m} \int_{V^{(m)}} \mathbf{H}^{(m)^{T}} \mathbf{f}^{B(m)} d V^{(m)}\right)+ \\
+ & \delta \hat{\mathbf{u}}^{T}\left(\sum_{m} \int_{S_{f}^{(m)}} \mathbf{H}^{(m) T} \mathbf{f}^{S}(m) d S^{(m)}\right)+ \\
- & \delta \hat{\mathbf{u}}^{T}\left(\sum_{m} \int_{V^{(m)}} \mathbf{B}^{(m) T} \boldsymbol{\sigma}^{I} d V^{(m)}\right)+ \\
+ & \delta \hat{\mathbf{u}}^{T}\left(\mathbf{r}_{C}\right)
\end{aligned}
$$

Os vetores de deslocamentos nodais $\mathbf{u}$ e deslocamentos virtuais nodais $\delta$ û não dependem do elemento $m$ e portanto podem ser tirados da somatória. De acordo com o PTV, os deslocamentos virtuais nodais $\delta$ û são arbitrários, portanto, pode-se escrever a Eq. (A.6) (BATHE, 2006)

$$
\mathbf{K u}=\mathbf{f}
$$

$\mathrm{Na}$ Eq. (A.6), K é a matriz de rigidez (ver Eq. (A.7) ) e f é o vetor de forças onde estão incluídos os efeitos das forças de volume, forças superficiais, tensões iniciais e cargas concentradas (ver Eqs. (A.8) a (A.11) ) (BATHE, 2006)

$$
\begin{gathered}
\mathbf{K}=\sum_{m} \int_{V^{(m)}} \mathbf{B}^{(m)^{T}} \mathbf{C}^{(m)} \mathbf{B}^{(m)} d V \\
\mathbf{f}=\mathbf{r}_{B}+\mathbf{r}_{S}-\mathbf{r}_{I}+\mathbf{r}_{C} \\
\mathbf{r}_{B}=\sum_{m} \int_{V^{(m)}} \mathbf{H}^{(m)} \mathbf{f}^{B(m)} d V^{(m)} \\
\mathbf{r}_{S}=\sum_{m} \int_{S_{f}^{(m)}} \mathbf{H}^{(m) T} \mathbf{f}^{S_{f}(m)} d S^{(m)} \\
\mathbf{r}_{I}=\sum_{m} \int_{V^{(m)}} \mathbf{B}^{(m) T} \boldsymbol{\sigma}^{I} d V^{(m)}
\end{gathered}
$$




\section{APÊNDICE B - IMPLEMENTAÇÃO DO FILTRO DE HELMHOLTZ}

Para implementar o filtro de Helmholtz, considera-se que a densidade em cada elemento é aproximada por meio da Eq. (B.1) (LAZAROV; SIGMUND, 2011)

$$
\tilde{a}^{(m)}=\mathbf{N}^{(m)} \hat{\mathbf{a}}^{(m)}
$$

onde â é o vetor de variáveis de projetos nodais genéricas. Considerando que a função de teste $v$ é igual a matriz de interpolação $\mathbf{H}^{(m)}$ e substituindo Eq. (B.1) em Eq. (3.8) tem-se um sistema de equações lineares para os valores de â que pode ser solucionado utilizando-se o MEF (ver Eqs. (B.2) a (B.4))

$$
\begin{gathered}
\mathbf{K}_{f} \hat{\mathbf{a}}=\mathbf{p}_{f} \\
\mathbf{K}_{f}=\sum_{m} \int_{V^{(m)}}\left(r^{2} \nabla \mathbf{N}^{(m)^{T}} \nabla \mathbf{N}^{(m)}+\mathbf{N}^{(m)^{T}} \mathbf{N}^{(m)}\right) d V^{(m)} \\
\mathbf{p}_{f}=\sum_{m} \int_{V^{(m)}} \mathbf{N}^{(m)^{T}} a d V^{(m)}
\end{gathered}
$$

